\title{
How clustered protocadherin binding specificity is tuned for neuronal self/non-self-recognition
}

\author{
Kerry M. Goodman ${ }^{1,7}$, Phinikoula S. Katsamba ${ }^{1,7}$, Rotem Rubinstein ${ }^{5,6}$ \\ Göran Ahlsén ${ }^{1}$, Fabiana Bahna ${ }^{1}$, Seetha Mannepalli ${ }^{1}$, Hanbin Dan ${ }^{4}$, \\ Rosemary Sampogna ${ }^{4}$, Lawrence Shapiro ${ }^{1,2^{*}}$, and Barry Honig ${ }^{1,2,3,4^{*}}$
}

${ }^{1}$ Zuckerman Mind, Brain and Behavior Institute, Columbia University, New York, NY 10027, USA

${ }^{2}$ Department of Biochemistry and Molecular Biophysics, Columbia University, New York, NY 10032, USA

${ }^{3}$ Department of Systems Biology, Columbia University, New York, NY 10032, USA

${ }^{4}$ Department of Medicine, Division of Nephrology, Columbia University, New York, NY 10032, USA

${ }^{5}$ School of Neurobiology, Biochemistry and Biophysics, Tel Aviv University, Israel

${ }^{6}$ Sagol School of Neuroscience, Tel Aviv University, Israel

${ }^{7}$ Equal contribution

*Correspondence: 1ss8@columbia.edu (L.S.); bh6@cumc.columbia.edu (B.H.) 


\section{$1 \quad$ Abstract (152 words)}

2 The stochastic expression of fewer than 60 clustered protocadherin (cPcdh) isoforms provides

3 diverse identities to individual vertebrate neurons and a molecular basis for self/non-self-

4 discrimination. cPcdhs form chains mediated by alternating cis and trans interactions between

5 apposed membranes, which has been suggested to signal self-recognition. Such a mechanism

6 requires that $\mathrm{cPcdh}$ cis dimers form promiscuously to generate diverse recognition units, and that

7 trans interactions have precise specificity so that isoform mismatches terminate chain growth.

8 However, the extent to which cPcdh interactions fulfill these requirements has not been

9 definitively demonstrated. Here we report biophysical experiments showing that cPcdh cis

10 interactions are promiscuous, but with preferences favoring formation of heterologous cis dimers.

11 Trans-homophilic interactions are remarkably precise, with no evidence for heterophilic

12 interactions between different isoforms. A new C-type cPcdh crystal structure and mutagenesis

13 data help to explain these observations. Overall, the interaction characteristics we report for cPcdhs

14 help explain their function in neuronal self/non-self-discrimination. 
16 Clustered protocadherins (cPcdhs) are a large family of cadherin-like proteins named for the 17 clustered arrangement of their genes in vertebrate genomes (Wu and Maniatis, 1999; Wu et al., 18 2001). cPcdhs play roles in many facets of neural development (Peek et al., 2017), including circuit 19 development, most notably neurite self-avoidance in vertebrates (Kostadinov and Sanes, 2015; 20 Lefebvre et al., 2012; Mountoufaris et al., 2017), and tiling (Chen et al., 2017). In self-avoidance, 21 neurites from the same neuron (sister neurites) actively avoid one another, whereas neurons from 22 different neurons can freely interact. Tiling is similar to self-avoidance, but in tiling all neurons 23 acquire the same identity, so that there is uniform repulsion among self- and non-self neurites

24 (Chen et al., 2017). Self-avoidance among sister neurites leads to the characteristic arbor structures 25 of dendritic trees, and prevents the formation of self-synapses (Kostadinov and Sanes, 2015; 26 Lefebvre et al., 2012).

The molecular mechanisms through which neurons discriminate self from non-self, differ between vertebrate and most invertebrate animals. For arthropod invertebrates such as Drosophila melanogaster, self-avoidance is mediated by immunoglobulin superfamily Dscam1 cell surface proteins. The stochastic alternative splicing of Dscaml pre-mRNAs can, in principle, generate

3219,008 distinct extracellular isoforms; the vast majority of which, based on ELISA-based binding 33 assay, mediate homophilic recognition (Miura et al., 2013; Schmucker et al., 2000; Wojtowicz et 34 al., 2004; Wojtowicz et al., 2007). Each neuron expresses a repertoire estimated at 10-50 isoforms 35 and the large number of Dscam1 isoforms ensures a low probability that any two contacting neurons will have an identical or even a similar isoform repertoire thus minimizing the chance of

37 inappropriate avoidance between non-self neurons (Hattori et al., 2009).

In mammalian nervous systems $\mathrm{cPcdh}$ isoform expression is controlled by the unique organization of three tandem gene clusters, $P c d h \alpha, P c d h \beta$, and $P c d h \gamma$ (Wu and Maniatis, 1999), with each cluster containing multiple variable exons, which encode full $\mathrm{cPcdh}$ ectodomain regions with six extracellular cadherin (EC) domains, a single transmembrane region, and a short cytoplasmic

43 extension (Figure 1A). The $P c d h \alpha$ and $P c d h \gamma$ gene clusters also contain three 'constant' exons that 44 encode cluster-specific intracellular domains. The last two variable exons in the Pcdh $\alpha$ gene 
45 cluster and the last three variable exons of the $P c d h \gamma$ gene cluster diverge in sequence from other

46 cPcdh isoforms and are referred to as 'C-type' cPcdhs (Wu and Maniatis, 1999; Wu et al., 2001).

47 Sequence differences further subdivide $P c d h \gamma$ genes into two subfamilies $-P c d h \gamma A$ and $P c d h \gamma B$

48 (Wu and Maniatis, 1999). The full mouse cPcdh complement is comprised of 53 non-C-type

49 cPcdhs, commonly known as alternate cPcdhs ( $\alpha 1-12, \beta 1-22, \gamma \mathrm{A} 1-12$, and $\gamma \mathrm{B} 1-7)$, whose

50 expression choices vary stochastically between cells through alternate promoter choice (Canzio

51 and Maniatis, 2019); and $5 \mathrm{C}$-type cPcdhs ( $\alpha \mathrm{C} 1, \alpha \mathrm{C} 2, \gamma \mathrm{C} 3, \gamma \mathrm{C} 4$, and $\gamma \mathrm{C} 5)$, which are constitutively

52 expressed. cPcdh expression, either stochastic or constitutive, varies between cell types: For

53 example, olfactory sensory neurons express $\sim 5-10$ cPcdhs stochastically; Purkinje neurons express

$54 \sim 10$ alternate cPcdhs stochastically and all five C-types constitutively (Esumi et al., 2005; Kaneko

55 et al., 2006); and serotonergic neurons express just $\alpha C 2$ constitutively (Canzio and Maniatis, 2019;

56 Chen et al., 2017). While the cPcdh and Dscam1 systems bear striking similarities, the relatively

57 small number of cPcdh isoforms - fewer than 60 - has presented a significant challenge to

58 generation of sufficient diversity to provide mammalian neurons with functionally unique

59 identities.

Solution biophysics and functional mutagenesis studies, have shown that cPcdhs interact in trans through antiparallel interactions between their EC1-EC4 regions (Rubinstein et al. 2015), and crystal structures of alternate $\alpha, \beta$, and $\gamma \mathrm{cPcdh}$ trans-homodimers have revealed interfaces 64 involving EC1 interacting with EC4 and EC2 with EC3 (Figure 1B) (Goodman et al., 2016a; 65 Goodman et al., 2016b; Nicoludis et al., 2016; Rubinstein et al., 2015; Thu et al., 2014). cPcdhs 66 also form cis dimers through their membrane-proximal EC5-EC6 regions, and are presented on 67 cell surfaces as cis dimers (Goodman et al., 2017; Rubinstein et al., 2015; Thu et al., 2014). Crystal structures of cis-interacting protocadherin ectodomains (Brasch et al., 2019; Goodman et al., 2017)

69 have revealed an asymmetrical interaction mode, where one molecule interacts through elements 70 of EC5 and EC6, and the other interacts exclusively through EC6 (Figure 1C). To date, structural 71 studies of C-type cPcdh interactions have not been available. Here we extend our molecular 72 understanding of cPcdhs to C-type isoforms as well, with the goal of understanding the 73 evolutionary design of the entire family. 
75 In order to explain how about $60 \mathrm{cPcdh}$ isoforms can provide a comparable or even greater level

76 of neuronal diversity as 19,000 Dscam isoforms, Rubinstein et al. (2015) proposed that cPcdhs

77 located on apposed membrane surfaces would form an extended zipper-like lattice through

78 alternating cis and trans interactions (Figure 1D). In self-interactions - between two membranes

79 with identical cPcdh repertoires - these chains would grow to form large structures, limited mainly

80 by the number of molecules (Brasch et al., 2019; Rubinstein et al., 2015). However, in non-self-

81 interactions - between two membranes with differing cPcdh repertoires - such large linear

82 assemblies would not form since even a single mismatch between expressed isoforms would

83 terminate chain assembly (Brasch et al., 2019; Rubinstein et al., 2017; Rubinstein et al., 2015).

84 This "isoform-mismatch chain-termination model" for the "barcoding" of vertebrate neurons

85 envisions the assembly of long cPcdh chains between sites of neurite-neurite contact to represent

86 the signature of "self", which is then translated by downstream signaling that leads to self-

87 avoidance (Fan et al., 2018). X-ray crystallographic studies and cryo-electron tomography (cryo-

88 ET) studies of the full-length $\mathrm{cPcdh}$ ectodomains bound between the surfaces of adherent

89 liposomes revealed the existence of linear zippers thus providing strong evidence supporting the

90 validity of the model (Brasch et al., 2019). However, crucial questions remain unanswered. Here,

91 a number of them are addressed.

93 1) For the proposed mechanism to successfully explain neuronal barcoding, cis interactions must

94 be promiscuous to generate diverse repertoires of cis-dimeric biantennary 'interaction units', while

95 trans interactions must be highly specific so that mismatched isoforms do not inappropriately

96 enable growth of the chain through heterophilic interactions. While cell aggregation assays have

97 suggested trans homophilic specificity, these assays only reflect a competition between different

98 cell populations and thus don't inform as to the strength of heterophilic interactions. Moreover,

99 the results of cell aggregation assays depend critically on the relative strengths of homophilic and

100 heterophilic interactions and thus do not inform as to actual binding affinities (Honig and Shapiro,

101 2020). It is thus necessary to establish the extent to which heterophilic trans interactions are truly

102 disallowed.

103

104 2) The assumption that cis interactions are promiscuous is based in large part on the fact that $\alpha$ 105 cPcdhs and $\gamma \mathrm{C} 4$ cannot reach the cell surface without binding in cis to another "carrier" isoform 
106 (Bonn et al., 2007; Goodman et al., 2016b; Murata et al., 2004; Schreiner and Weiner, 2010; Thu 107 et al., 2014). As is the case for trans interactions, the strength of cis interactions has only been 108 probed quantitatively in a small number of cases so that the term "promiscuous" is qualitative at 109 best. In fact, as compared to $\gamma \mathrm{B}$ and $\beta \mathrm{cPcdh}$ isoforms, most $\gamma \mathrm{A}$-Pcdhs do not form measurable cis 110 homodimers in solution (Goodman et al., 2016b) (Figure 4-source data 1). Nevertheless, all $\gamma \mathrm{A}$ 111 Pcdhs are still able to reach the cell surface when expressed alone (Thu et al., 2014). This 112 observation can be understood if the cis dimerization affinity of $\gamma \mathrm{A}-\mathrm{Pcdh}$ is large enough to enable 113 them to dimerize in the 2D membrane environment (Goodman et al., 2016b; Wu et al., 2013). 114 Nevertheless, their weak dimerization affinities suggest, more generally, that cPcdhs may exhibit 115 a range of cis dimerization affinities. We establish below that a wide range of affinities does in 116 fact exist and, strikingly, most homophilic cis interactions are weaker than their heterophilic 117 counterparts. We consider the functional implications of this novel observation in the discussion.

119 3) Structures have not yet been determined for complete C-type cPcdh ectodomains. Yet these 120 isoforms play unique functional roles, some of which have no apparent connection to isoform 121 diversity. For example, a single C-type isoform is sufficient for tiling which can be simply 122 understood in terms of the formation of zippers containing identical homodimers so that all 123 interacting neurons will avoid one another (Chen et al., 2017). Moreover, Garrett and coworkers 124 discovered that neuronal survival and postnatal viability is controlled solely by $\gamma \mathrm{C} 4$ suggesting a 125 function that is unique to this isoform (although it presumably requires $\beta$ and/or other $\gamma$ carriers to 126 reach the cell surface) (Garrett et al., 2019). Additionally, a recent paper by Iqbal and coworkers 127 has shown that genetic $\gamma \mathrm{C} 4$ variants cause a neurodevelopmental disorder which is potentially 128 linked to $\gamma \mathrm{C} 4$ 's role in programmed cell death of neuronal cells (Iqbal et al., 2021). Below we 129 report extensive biophysical interaction studies of C-type isoform ectodomains and report the first 130 crystal structure of a trans dimer formed by $\gamma \mathrm{C} 4$. Our findings reveal that the specialized functions 131 of C-type cPcdhs probably do not involve unique structural or biophysical properties of their 132 ectodomains.

134 Overall, in accordance with the requirements of the isoform-mismatch chain-termination model, 135 we find that trans-homophilic interactions are remarkably precise, with no evidence for 136 heterophilic interactions between different $\mathrm{cPcdh}$ isoforms. In contrast $\mathrm{cPcdh}$ cis interactions are 
137 largely promiscuous but with relatively weak intra-subfamily and, especially, homophilic 138 interactions. Possible implications of this somewhat surprising finding are considered in the 139 discussion. Our study reveals how the extraordinary demands posed by the need to assign each 140 neuron with a unique identity are met by an unprecedented level of protein-protein interaction 141 specificity.

\section{Results}

\section{cPcdh trans interactions are strictly homophilic}

145 We generated biotinylated ectodomain fragments containing the trans-interacting EC1-4 regions 146 (Nicoludis et al., 2015; Rubinstein et al., 2015) of six representative $\alpha, \beta, \gamma \mathrm{A}$, and $\gamma \mathrm{B}$ mouse cPcdh 147 isoforms $-\alpha 7, \beta 6, \beta 8, \gamma \mathrm{A} 8, \gamma \mathrm{A} 9$ and $\gamma \mathrm{B} 2-$ which include the most closely related isoforms by 148 sequence identity from the $\beta$ and $\gamma$ A subfamilies ( $\beta 6 / 8$ and $\gamma$ A8/9) (Rubinstein et al., 2015). These 149 molecules were coupled over independent Neutravidin-immobilized flow cells and trans150 interacting ectodomain fragments of multiple members of each cPcdh subfamily, including the C151 types $(\alpha 4, \alpha 7, \alpha 12, \beta 6, \beta 8, \gamma \mathrm{A} 4, \gamma \mathrm{A} 8, \gamma \mathrm{A} 9, \gamma \mathrm{B} 2, \gamma \mathrm{B} 4, \gamma \mathrm{B} 5, \alpha \mathrm{C} 2, \gamma \mathrm{C} 3, \gamma \mathrm{C} 4$, and $\gamma \mathrm{C} 5)$, were then 152 flowed over the six cPcdh surfaces to assess their binding. The surface plasmon resonance (SPR) 153 binding profiles reveal strictly homophilic binding (Figure 2A). All ectodomain fragments used 154 in these SPR experiments were confirmed to form homodimers in solution by sedimentation 155 equilibrium analytical ultracentrifugation (AUC) (Figure 2-source data 1), validating that these

156 proteins are well-behaved and active. Remarkably, no heterophilic binding was observed for any 157 of the analytes over any of the six surfaces (Figure $2 A$ ). Even $\beta 6 / 8$ and $\gamma A 8 / 9$ that have $92 \%$ and $15882 \%$ sequence identities respectively in their trans-binding EC1-4 regions exhibit no heterophilic 159 binding. We estimate that, for heterophilic trans-dimers, the lower limit for the dissociation 160 constant $\left(\mathrm{K}_{\mathrm{D}}\right)$ would be $\sim 200 \mu \mathrm{M}$. Mutations designed to disrupt $\alpha 7, \beta 6$, and $\gamma \mathrm{A} 8$ trans interaction 161 inhibited homophilic binding, demonstrating that the observed binding occurs via the trans 162 interface (Figure 2-figure supplement 1A) (Goodman et al., 2016a; Goodman et al., 2016b; 163 Rubinstein et al., 2015). This behavior is unlike that of other adhesion receptor families where, 164 whether they display homophilic or heterophilic preferences, the signal is never as binary as the 165 one shown in Figure 2 (Honig and Shapiro, 2020). 
167 Much of the original evidence as to homophilic specificity was based on cell aggregation assays 168 (Rubinstein et al., 2015; Schreiner and Weiner, 2010; Thu et al., 2014) and it is of interest to 169 compare the results obtained from these assays to those obtained from SPR. We do this in the 170 context of examining the heterophilic binding specificity between $\beta 6_{1-4}$ and $\beta 8_{1-4}$ trans fragments 171 that share 92\% sequence identity and differ at only five residues (Figure 2-figure supplement 172 2A), within their respective binding interfaces (Goodman et al., 2016a). Each of these residues 173 was mutated individually and in combination. Figure 2 - figure supplement $2 \mathrm{~B}$ and C display SPR 174 profiles and cell aggregation images, respectively, for wild type $\beta 6$ and $\beta 8$ and for the various 175 mutations. We first note that changing all five residues in $\beta 6$ to those of $\beta 8$ generates a mutant 176 protein with essentially wild type $\beta 8$ properties; it binds strongly to $\beta 8$ but not to $\beta 6$ as seen in

177 SPR and also forms mixed aggregates with $\beta 8$ but not $\beta 6$. In contrast, most of the single residue 178 mutants retain $\beta 6$-like properties in both assays whereas double and triple mutants exhibit 179 intermediate behavior between $\beta 6$ and $\beta 8$. These results demonstrate that despite the $92 \%$ sequence 180 identity between $\beta 6$ and $\beta 8$, their highly specific homophilic properties can be attributed to five 181 interfacial residues. Moreover, the cell aggregation assays are consistent with the heterophilic 182 binding traces measured by SPR; cells expressing mutants that generate strong SPR signals with 183 either wild type $\beta 6$ or $\beta 8$ also form mixed aggregates with cells expressing the same wild-type 184 protein.

186 Of note, trans-interacting fragments of all four C-type cPcdhs tested showed no binding over the 187 alternate cPcdh SPR surfaces (Figure 2A). To test whether C-type cPcdhs also show strict 188 homophilic specificity with respect to each other we coupled biotinylated trans-interacting 189 fragments of $\alpha \mathrm{C} 2, \gamma \mathrm{C} 3, \gamma \mathrm{C} 4$, and $\gamma \mathrm{C} 5$ to SPR chips and passed the same four fragments alongside 190 alternate cPcdh trans fragments over these four surfaces. Only homophilic binding was observed, 191 with each of the four C-type fragments binding to its cognate partner and no other isoform (Figure 192 2B). Disrupting the $\gamma \mathrm{C} 5$ trans interaction with the S116R mutation (Rubinstein et al., 2015), 193 inhibited binding to the $\gamma \mathrm{C} 5$ surface, demonstrating that the observed binding occurs via the trans 194 interface (Figure 2-figure supplement 1B). 
196 In contrast to the other C-type isoforms, $\alpha \mathrm{C} 1$ does not mediate cell-cell interactions in cell 197 aggregation assays even when co-expressed with cPcdhs that facilitate cell-surface delivery of $\gamma \mathrm{C} 4$

198 (Thu et al., 2014). Although we have been able to produce an $\alpha \mathrm{C} 1 \mathrm{EC} 1-4$ fragment the 199 recombinant molecule forms disulfide-linked multimers which are likely non-native, precluding 200 confident examination of $\alpha \mathrm{C} 1$ 's potential trans interactions. Notably, the sequence of mouse $\alpha \mathrm{C} 1$ 201 reveals the EC3:EC4 linker does not contain the full complement of calcium-coordinating residues, 202 which may impact the structure and binding properties of this protein (Thu et al., 2014).

204 Since all the cPcdh trans fragment molecules used in these SPR experiments homodimerize our 205 SPR data cannot be used to determine accurate binding affinities (Rich and Myszka, 2007). We 206 therefore used AUC to measure the trans-homodimer $\mathrm{K}_{\mathrm{DS}}$ (Figure 2-source data 1) revealing a $207>200$-fold range of binding affinities, from $2.9 \mu \mathrm{M}\left(\alpha 7_{1-5}\right)$ to $>500 \mu \mathrm{M}\left(\gamma \mathrm{C}_{1-4}\right)$. Regardless of 208 their trans binding affinity, all cPcdhs (except $\alpha \mathrm{C} 1$ ) have previously been shown to effectively 209 mediate cell-cell interactions in cell aggregation assays (Schreiner and Weiner, 2010; Thu et al., 210 2014).

\section{Crystal structure of C-type cPcdh $\gamma$ C4 reveals EC1-4-mediated head-to-tail trans dimer interaction}

213 The biophysical properties of C-type cPcdhs pose a number of interesting questions: Despite their 214 more divergent sequences compared with alternate cPcdhs, AUC data has confirmed that C-type $215 \mathrm{cPcdhs} \alpha \mathrm{C} 2, \gamma \mathrm{C} 3$, and $\gamma \mathrm{C} 5$ form trans-dimers using their EC1-4 domains (Goodman et al., 2016b;

216 Rubinstein et al., 2015). However, $\gamma \mathrm{C}_{1-4}$ behaved as a very weak dimer in $\mathrm{AUC}\left(\mathrm{K}_{\mathrm{D}}>500 \mu \mathrm{M}\right.$;

217 Figure 2-source data 1), nevertheless full-length $\gamma \mathrm{C} 4$ can mediate cell aggregation when 218 delivered to the cell surface by co-expression with a 'carrier' cPcdh (Thu et al., 2014). In addition,

219 C-type isoforms have unique expression profile and function compared to alternate cPcdhs (Canzio 220 and Maniatis, 2019; Mountoufaris et al., 2016). However, there are no published crystal structures 221 of C-type cPcdh trans dimers. We therefore sought to crystallize a C-type $\mathrm{cPcdh}$ engaged in a trans 222 interaction and obtained two distinct crystal forms of $\gamma \mathrm{C} 4_{\mathrm{ECl}-4}$, one at $2.4 \AA$ resolution (crystallized 223 at $\mathrm{pH}$ 7.5) and the other with anisotropic diffraction at 4.6/3.9/3.5 $\AA$ resolution (Figure 3A, Figure 224 3-figure supplement 1A,B, Figure 3-source data 1) (crystallized at pH 6.0). Both crystal 225 structures revealed an EC1-4-mediated head-to-tail trans dimer: The 4.6/3.9/3.5 $\AA$ crystal 
226

227

228

229

230

231

232

233

234

235

236

237

238

239

240

241

242

243

244

245

246

247

248

249

250

251

252

253

254

structure appears to have a fully intact trans interface with a total buried surface area of $3800 \AA^{2}$, which is a similar size to other cPcdh trans dimer interfaces (Goodman et al., 2016a; Goodman et al., 2016b; Nicoludis et al., 2016) (Figure 3B, Figure 3-figure supplement 1B). However, the 2.4 $\AA$ structure had an apparently partially disrupted EC2:EC3 interface resulting in a total buried surface area of just $2900 \AA^{2}$ (Figure 3B). The difference between the two structures may be due to differences in the $\mathrm{pH}$ of the crystallization and its effect on the ionization state of the three histidines present in the EC2:EC3 interface (Figure 3B). The differences could also reflect distinct states of a dynamic interaction, as has previously been observed crystallographically (Nicoludis et al., 2016; Goodman et al., 2016b) and explored computationally for other cPcdh trans interactions (Nicoludis et al., 2019).

Despite the $\gamma \mathrm{C} 4$ trans dimer sharing structural similarity and the interface having similar buried surface area as alternate $\alpha, \beta, \gamma \mathrm{A}$, and $\gamma \mathrm{B} \mathrm{cPcdhs}$ and $\delta 2$ non-clustered Pcdhs (Figure 3-source data 2) (Cooper et al., 2016; Goodman et al., 2016a; Goodman et al., 2016b; Harrison et al., 2020; Hudson et al., 2021; Nicoludis et al., 2016), its binding affinity is very weak. The two most structurally similar molecules to $\gamma \mathrm{C} 4$ over their trans interacting domains, cPcdh $\gamma \mathrm{B} 2$ and nonclustered Pcdh19. $\gamma \mathrm{B} 2$ and Pcdh19 have trans dimer $\mathrm{K}_{\mathrm{DS}}$ of $21.8 \mu \mathrm{M}$ and $0.48 \mu \mathrm{M}$ respectively (Harrison et al., 2020), while that of $\gamma \mathrm{C} 4$ is $>500 \mu \mathrm{M}$. Comparison between the $\gamma \mathrm{B} 2$ and $\gamma \mathrm{C} 4$ dimer interfaces highlighted two buried charges in the $\gamma \mathrm{C} 4$ trans interface, E78 and D290, which could potentially contribute to the low interaction affinity (Figure 3C). To test this, we mutated these two residues to neutral amino acids and used AUC to determine whether the binding affinity increased: The two D290 mutations we tested, D290A and D290N, had no measurable impact on binding; but mutating E78 significantly increased the binding affinity with $\gamma \mathrm{C} 4_{\mathrm{EC} 1-4} \mathrm{E} 78 \mathrm{~A}$ showing a $\mathrm{K}_{\mathrm{D}}$ of $58 \mu \mathrm{M}$ and $\gamma \mathrm{C} 4_{\mathrm{ECl}-4} \mathrm{E} 78 \mathrm{Q}, 83 \mu \mathrm{M}$ (Figure 3D, Figure 3-figure supplement 1C). The equivalent residue to E78 in $\gamma \mathrm{B} 2$ is also charged (D77) and forms a salt bridge with K340 in the $\gamma \mathrm{B} 2$ dimer (Figure 3C). To assess whether generating a similar salt bridge in $\gamma \mathrm{C} 4$ would compensate for the negative impact of E78 on dimer affinity we generated an S344R mutant. Similar to the E78 mutants, $\gamma \mathrm{C}_{\mathrm{EC} 1-4} \mathrm{~S} 344 \mathrm{R}$ also had a stronger binding affinity than wild type with a $\mathrm{K}_{\mathrm{D}}$ of $112 \mu \mathrm{M}$ (Figure 3D, Figure 3-figure supplement 1C). It appears then that E78 plays 
an important role in weakening cPcdh $\gamma \mathrm{C} 4$ 's trans interaction although the functional reasons for $\gamma \mathrm{C} 4$ 's weak trans interaction are unclear.

\section{Clustered protocadherin cis interactions are promiscuous with a range of interaction strengths}

259 To systematically investigate cPcdh cis interactions, we coupled cis-interacting fragments of $\beta 9$, $\gamma \mathrm{A} 4, \gamma \mathrm{A} 9, \gamma \mathrm{B} 2, \alpha \mathrm{C} 2, \gamma \mathrm{C} 3$, and $\gamma \mathrm{C} 5$ to SPR chip surfaces. Cis-interacting fragments of three members from each of the $\beta, \gamma \mathrm{A}$, and $\gamma \mathrm{B}$ subfamilies $(\beta 1, \beta 6, \beta 9, \gamma \mathrm{A} 3, \gamma \mathrm{A} 4, \gamma \mathrm{A} 9, \gamma \mathrm{B} 2, \gamma \mathrm{B} 5, \gamma \mathrm{B} 7)$

262 alongside $\alpha \mathrm{C} 2, \gamma \mathrm{C} 3$, and $\gamma \mathrm{C} 5$ fragments were flowed over the seven surfaces to detect their 263 heterophilic binding (Figure 4A). Alternate $\alpha$-cPcdhs, and the C-types $\alpha \mathrm{C} 1$ and $\gamma \mathrm{C} 4$ were not 264 included in this study since EC6-containing fragments of these molecules cannot be expressed, 265 although an $\alpha 7_{\mathrm{EC} 1-5} / \gamma \mathrm{C} 3_{\mathrm{EC} 6}$ chimera was included among the analytes to assess the role of $\alpha 7 \mathrm{EC} 5$

266 (Figure 4-figure supplement 1C). Each of the analytes was also analyzed by AUC to determine 267 their homophilic cis-interaction behavior (Figure 4 - source data 1): Four analytes, $\beta 1_{3-6}, \gamma \mathrm{A}_{3_{-}}$ 6, $\gamma \mathrm{A}_{3_{3-6}}$, and $\gamma \mathrm{C} 3_{3-6}$, are monomeric in solution as measured by $\mathrm{AUC}$, therefore their SPR binding profiles could be analyzed to determine their heterophilic binding affinities (Figure 4B, Figure 4-

270 figure supplement 1A,B). For the remaining analytes, due to the added complexity of their

271 homophilic cis interactions in solution competing with their binding to the immobilized molecules, 272 the SPR responses could not be analyzed to determine accurate $K_{D S}$ (Rich and Myszka, 2007).

274 The data clearly demonstrate a wide range of cis dimerization affinities with strong heterophilic 275 binding signals (500-2000 RU), with much weaker homophilic binding responses typically 276 between 100-140 RU. The strongest heterophilic cis interactions are in the sub-micromolar range; 277 for example, $\gamma \mathrm{C} 3 / \beta 9$ can heterophilically cis-dimerize with a $\mathrm{K}_{\mathrm{D}}$ of $0.22 \mu \mathrm{M}$, while $\beta 9_{3-6}, \gamma \mathrm{B} 2_{3-6}$, $278 \alpha \mathrm{C} 2_{2-6}$ and $\gamma \mathrm{C} 5_{2-6}$ homodimerize with AUC-determined $\mathrm{K}_{\mathrm{DS}} \mathrm{s}$ of 9-80 $\mu \mathrm{M}$. In addition to uniformly 279 weak homophilic interactions, within-subfamily cis interactions were consistently among the 280 weakest observed although a number of inter-subfamily interactions were also relatively weak 281 (Figure 4A). For example, for the $\beta 9$ surface comparatively weak binding was observed for all 282 tested $\beta$ and $\gamma \mathrm{A}$ isoforms except $\gamma \mathrm{A} 3$, with the monomeric $\beta 1, \gamma \mathrm{A} 4$ and $\gamma \mathrm{A} 9$ producing low 283 responses that could not be fit to a binding isotherm to calculate accurate $\mathrm{K}_{\mathrm{D}} \mathrm{S}$ (Figure 4B, Figure $284 \quad 4$-figure supplement 1B). In contrast, robust binding to the $\beta 9$ surface was observed for all $\gamma \mathrm{B}$ 
and C-type isoforms. These data are consistent with the binding responses when $\beta 9$ was used as an analyte over the other six surfaces, with weak to no binding observed over the $\gamma \mathrm{A} 4$ and $\gamma \mathrm{A} 9$ surfaces and robust responses over the $\gamma \mathrm{B} 2, \alpha \mathrm{C} 2, \gamma \mathrm{C} 3$, and $\gamma \mathrm{C} 5$ surfaces (Figure 4A). The $\gamma \mathrm{A} 4$ and $\gamma$ A9 surfaces showed a similar pattern of binding behaviors, with weak to no binding observed for the $\gamma \mathrm{A}$ and $\alpha \mathrm{C} 2$ analytes, and robust binding for the $\gamma \mathrm{C}$-cPcdhs with $\mathrm{K}_{\mathrm{DS}}$ for $\gamma \mathrm{C}_{3-6}$ of 2.73 and $9.60 \mu \mathrm{M}$ respectively over each surface (Figure 4, Figure 4-figure supplement 1B).

292 Overall, these SPR data show that cPcdh cis binding is generally promiscuous, with measurable 293 cis interactions observed for $86 \%$ of pairs tested (using a 40 RU threshold). However, the wide range 294 of binding responses and homo- and hetero-dimeric $\mathrm{K}_{\mathrm{DS}}$ that span $0.2201 \mu \mathrm{M}$ to no measurable 295 interaction in solution suggests certain cis dimers will form preferentially to others. For the 296 heterophilic binding pairs for which $\mathrm{K}_{\mathrm{DS}}$ could be determined (Figure 4B, Figure 4-figure 297 supplement 1, Figure 4-figure supplement 2), the alternate cPcdhs in particular, form markedly 298 stronger cis heterodimers with members of different subfamilies, particularly $\gamma \mathrm{C} 3$ and/or $\gamma \mathrm{C} 5$, compared to their homodimeric and within-subfamily interactions. $\gamma \mathrm{C} 3$ also formed stronger heterodimers with $\alpha \mathrm{C} 2$ than with itself or $\gamma \mathrm{C} 5$. Of note, $\alpha \mathrm{C} 2$ and $\gamma \mathrm{C} 5$ both form strong cis

301 homodimers with $\mathrm{K}_{\mathrm{DS}}$ of $8.9 \mu \mathrm{M}$ and $18.4 \mu \mathrm{M}$ respectively as determined from AUC experiments 302 (Figure 4-source data 1), a magnitude similar to many of their heterodimeric interactions of 11.5 $\mu \mathrm{M}$ and 6.9-18.2 $\mu \mathrm{M}$ respectively (Figure 4B).

In the next section we rationalize cis binding preferences in terms of the structural properties of cis dimers.

\section{The asymmetric cis dimer interface and cis binding specificity}

309 The crystal structure of the $\gamma \mathrm{B} 7$ cis dimer revealed an asymmetric interaction, with the dimer 310 formed by one protomer engaging using surface of both EC5 and EC6 and one protomer engaging 311 using only EC6 (Goodman et al., 2017) with regions of EC6 overlapping in both EC5-6 and the 312 EC6-only interfaces for all cPcdh subfamilies (Thu et al., 2014; Goodman et al., 2017). The 313 asymmetric nature of the cis interaction implies that for each dimer interaction there are two 314 possible arrangements: one with protomer "1" forming the EC5-6 side and protomer " 2 " forming 
315 the EC6-only side and the second where protomer "1" forms the EC6-only side and "2" the EC5-

3166 side. These two configurations are distinct with different residue:residue interactions. Alternate

$317 \alpha$-cPcdhs, which can only form the EC5-6 side of the cis dimer, require co-expression with a

318 "carrier" cPcdh from another cPcdh subfamily, which can form the EC6-only side of the cis dimer,

319 for robust delivery to the cell surface (Thu et al., 2014, Goodman et al., 2017). Although $\alpha$-cPcdhs

320 and $\gamma \mathrm{C} 4$, which also requires a carrier for delivery to the cell surface, are likely to be extreme

321 cases, sequence analysis alongside the low homodimerization ability of many cPcdh isoforms

322 suggests many cPcdhs will more readily form one side of the cis interface than the other (Goodman

323 et al., 2017).

We previously suggested that $\gamma \mathrm{A}-\mathrm{cPcdh}$ will prefer to form the EC6-only side of the interface since they have a poorly conserved EC5 interface and do not form strong homodimers in solution

327 (Figure 4-source data 1) (Goodman et al., 2017). The C-type cPcdh $\gamma \mathrm{C} 3$ also does not form cis

328 homodimers in solution. However, as shown in Figure 4, $\gamma \mathrm{A}$-cPcdhs form strong heterodimers

329 with $\gamma \mathrm{C} 3$ with dissociation constants in the low-micromolar range (Figure 4B and Figure 4-figure

330 supplement 1B). Structure-guided sequence analysis for the $\gamma \mathrm{A} 4 / \gamma \mathrm{C} 3$ dimer in both EC6-only and

331 EC5-6 possible orientations, using the available crystal structures of the $\gamma \mathrm{B} 7_{\mathrm{EC} 3-6}$ cis dimer and

332 monomeric $\gamma \mathrm{A}_{\mathrm{EC} 3-6}$ (Figure 5A and Figure 5-figure supplement 1), suggests that $\gamma \mathrm{C} 3$ prefers to

333 form the EC5-6 side: $\gamma \mathrm{C} 3$ has a number of residue differences in interface residues that are

334 conserved among $\beta, \gamma \mathrm{A}$ and $\gamma \mathrm{B}$ cPcdhs (V/L555, R/K558, W/V562, and S/R595) that seem likely

335 to disfavor the EC6-only side of the interface and favor the EC5-6 side (Figure 5-figure

336 supplement 1B,C). Two of these residues, V555 and S595, result in a potential loss of EC6-only

337 interface buried surface area and are shared with $\alpha$-cPcdhs, which cannot occupy the EC6-only

338 position (Goodman et al., 2017). Structural analysis further suggests that $\gamma \mathrm{C} 3$-specific residue

339 R558 would not be well accommodated from the EC6-only side, potentially causing van der Waals

340 clashes (Figure 5-figure supplement 1C). By contrast, from the EC5-6 side R558 is positioned

341 to form an additional salt bridge with $\gamma \mathrm{A} 4$ residue E544 and a hydrogen bond with Y532,

342 promoting dimer formation (Figure 5A; Figure 5-figure supplement 1B). $\gamma \mathrm{A} 4$ residue E544 is

343 positioned to form this salt bridge due to the EC6 A/A' loop region adopting a different 
344 arrangement in the $\gamma \mathrm{A} 4$ crystal structure to that observed for $\gamma \mathrm{B} 2$ and $\gamma \mathrm{B} 7$ in their respective crystal structures (Goodman et al., 2016c; Goodman et al., 2017).

347 Based on our analysis, we generated mutants of both $\gamma \mathrm{A} 4$ and $\gamma \mathrm{C} 3$ targeting the EC6-only side of 348 the interface and used size exclusion-coupled multi-angle light scattering (SEC-MALS) to assess 349 their preferred orientation on $\gamma \mathrm{A} 4 / \gamma \mathrm{C} 3$ heterodimerization. In SEC-MALS wild type $\gamma \mathrm{A} 4_{\mathrm{EC} 3-6}$ and $350 \gamma \mathrm{C} 3_{\mathrm{EC} 3-6}$ behave as monomers when run alone, and form a dimer when mixed in equimolar 351 amounts (Figure 5B; Figure 5-figure supplement 2A). The V560R mutation $(\gamma \mathrm{B} 7$ numbering, see 352 methods for sequence alignment) is based on EC6-only impaired $\alpha$-cPcdhs, and has been 353 previously shown to block $\gamma$ B6's homophilic cis interaction in solution (Goodman et al., 2017). $354 \gamma \mathrm{A} 4 \mathrm{~V} 560 \mathrm{R}$ did not dimerize with wild-type $\gamma \mathrm{C} 3$, whereas $\gamma \mathrm{C} 3 \mathrm{~V} 560 \mathrm{R}$ could still dimerize with 355 wild type $\gamma \mathrm{A} 4$ (Figure 5B). Therefore impairing $\gamma \mathrm{A} 4$ 's EC6-only interface blocks $\gamma \mathrm{A} 4 / \gamma \mathrm{C} 3$ dimer 356 formation while impairing $\gamma \mathrm{C} 3$ 's EC6-only interface does not (although the dimerization appears 357 to be weaker compared to the wild type $\gamma \mathrm{A} 4 / \gamma \mathrm{C} 3$ cis interacting pairs). We also generated a $\gamma \mathrm{C} 3-$ 358 like mutant of $\gamma \mathrm{A} 4, \mathrm{~K} 558 \mathrm{R}$, which also targets the EC6-only interface. Like $\gamma \mathrm{A} 4$ V560R, $\gamma \mathrm{A} 4$ 359 K558R also did not dimerize with wild type $\gamma \mathrm{C} 3$ in MALS and, when replicated, in SPR 360 experiments (Figure 5B, Figure 5-figure supplement 2B). The reverse mutation in $\gamma \mathrm{C} 3$, R558K, 361 inhibited dimerization with wild type $\gamma \mathrm{A} 4$ (Figure 5B). Therefore, like the $\alpha$-specific R560 residue, $362 \gamma \mathrm{C} 3$-specific R558 has distinct effects on dimerization when in $\gamma \mathrm{A} 4$ or $\gamma \mathrm{C} 3$, inhibiting 363 heterodimerization when mutated into $\gamma \mathrm{A} 4$ but promoting heterodimerization in $\gamma \mathrm{C} 3$. Together 364 these data suggest that the $\gamma \mathrm{A} 4 / \gamma \mathrm{C} 3$ dimer has a preferred orientation, with $\gamma \mathrm{A} 4$ predominantly 365 occupying the EC6-only position and $\gamma \mathrm{C} 3$ the EC5-6 side. Our data also account for the fact that 366 neither isoform homodimerizes in solution since the EC5-6 side would be impaired in the $\gamma \mathrm{A} 4$ 367 homodimer while the EC6 side would be impaired in the $\gamma \mathrm{C} 3$ homodimer.

369 Next, we sought to test whether $\gamma \mathrm{A} 4$ and $\gamma \mathrm{C} 3$ preferentially adopt these specific positions in $c i s$ 370 interactions with a $\gamma \mathrm{B}$ isoform. To accomplish this we generated mutants of $\gamma \mathrm{B} 7$ individually 371 targeting the EC6-only interaction surface, $\gamma \mathrm{B} 7 \mathrm{Y532G}$, and the EC5-6 side, $\gamma \mathrm{B} 7$ A570R, 372 respectively (Goodman et al., 2017) (Figure 4-source data 1). In SPR, $\gamma$ B7 Y532G had only a 
373

small impact on $\gamma \mathrm{A} 4$ binding, while $\gamma \mathrm{B} 7 \mathrm{~A} 570 \mathrm{R}$ abolished $\gamma \mathrm{A} 4$ binding (Figure 5C). In contrast, $\gamma \mathrm{B} 7 \mathrm{Y} 532 \mathrm{G}$ prevented $\gamma \mathrm{C} 3$ binding while $\gamma \mathrm{B} 7 \mathrm{~A} 570 \mathrm{R}$ showed robust $\gamma \mathrm{C} 3$ binding (Figure 5C). These results suggest that $\gamma \mathrm{A} 4 / \gamma \mathrm{B} 7$ and $\gamma \mathrm{C} 3 / \gamma \mathrm{B} 7$ cis heterodimers also have preferred orientations with $\gamma \mathrm{A} 4$ and $\gamma \mathrm{C} 3$ maintaining their preferences for the EC6-only and EC5-6 positions respectively. Additionally, SPR data for the $\gamma \mathrm{B} 7$ mutants over the $\alpha \mathrm{C} 2$ surface suggests $\alpha \mathrm{C} 2$ preferentially occupies the EC6-only side in $\alpha \mathrm{C} 2 / \gamma \mathrm{B} 7$ dimers (Figure $5 \mathrm{C}$ ). This is notable since $\alpha \mathrm{C} 2$ forms robust $c i s$-homodimers and therefore, like $\gamma \mathrm{B} 7$, can presumably readily occupy both positions in its homophilic interactions, implying that the $\alpha \mathrm{C} 2 / \gamma \mathrm{B} 7$ orientation preference could be specific to the particular heterodimer pairing. However, since this interpretation is based on a single mutation further interrogation of $\alpha \mathrm{C} 2$ 's interactions would be required to be conclusive. A broader examination of orientation preferences among cis dimer pairings beyond those of molecules with weak cis homodimer affinities, such as $\gamma \mathrm{A} 4$ and $\gamma \mathrm{C} 3$ examined here, could be instructive.

\section{Discussion}

Trans specificity - The results of this study add to our current understanding of cPcdhs in a number of ways. First, they reveal a remarkable level of specificity in trans homophilic interactions in the sense that in no case was a heterophilic trans interaction detected in our SPR measurements. Prior data has clearly indicated that $\mathrm{cPcdhs}$ exhibit a preference for homophilic trans interactions but the extent of this specificity was not established in quantitative terms but were, rather, based on cell aggregation experiments. The SPR experiments with cPcdhs reported here show no evidence of cross-interaction between non-identical cPcdh isoforms. This level of specificity is unusual for cell-cell recognition proteins, as significant intra-family interactions are evident in most other families examined to date including type I cadherins (Katsamba et al., 2009; Vendome et al., 2014), type II cadherins (Brasch et al., 2018), DIPs and Dprs (Cosmanescu et al., 2018), sidekicks (Goodman et al., 2016c), and nectins (Harrison et al., 2012). Even the non-clustered $\delta$ protocadherins, which are preferentially homophilic and utilize an antiparallel EC1-4 interface like the cPcdhs (Cooper et al., 2016; Harrison et al., 2020; Modak and Sotomayor, 2019), show 
401 heterophilic intra-family trans interactions, though they show no cross-reactivity with cPcdhs

402 (Harrison et al., 2020).

404 High fidelity homophilic interaction is a strict requirement of the chain termination model for the 405 barcoding of vertebrate neurons and has been accomplished through the exploitation of a 406 multidomain interface of almost $4000 \AA^{2}$ (Nicoludis et al., 2019) that enables the positioning of 407 enough "negative constraints" (Sergeeva et al., 2020) to preclude the dimerization of about 1600 408 heterophilic pairs of 58 mouse cPcdh isoforms (Rubinstein et al., 2017). Dscams accomplish the 409 same task for thousands of isoforms by exploiting the combinatorics made possible by a three410 domain interface where each domain interacts largely independently with an identical domain on 411 its interacting partner (see discussion in (Zipursky and Grueber, 2013)). Although it is likely that 412 Dscams dimerize with a comparable level of homophilic specificity to that of cPcdhs, the evidence 413 is based on a semi-quantitative ELISA-type assay of recombinant multimerized isoforms 414 (Wojtowicz et al., 2007) and AUC experiments on a few select isoforms (Wu et al., 2012).

416 Cis interactions - Despite early evidence that cis interactions are promiscuous, the data reported 417 here indicate that this generalization needs to be significantly refined. Functional mutagenesis 418 studies have already established that alternate $\alpha$ cPcdhs and the C-type $\gamma \mathrm{C} 4$ do not form intra419 subtype cis interactions and can only reach the cell surface when mediated by heterophilic cis 420 interactions with members of other subtype families (Goodman et al., 2017; Thu et al., 2014). The 421 data presented in Figure 4 indicate that this is an extreme example of quite general behavior: intra422 subtype $c$ is interactions are invariably weaker than inter-subtype interactions. However, unlike $\alpha$ 423 cPcdhs, most cPcdhs can reach the cell surface on their own. This includes $\beta 1$, all $\gamma \mathrm{A}-\mathrm{Pcdhs}$, and $424 \gamma \mathrm{C} 3$ which do not form measurable homodimeric cis interactions in our solution-based AUC 425 experiments. We have attributed this to their presence on the restricted 2D surface of membranes 426 which can promote cis-dimerization (Wu et al., 2013) whereas biophysical experiments are carried 427 out in a 3D solution environment (Goodman et al., 2016b). (There may of course be other, still 428 undetermined, factors involved in cPcdh cell surface transport (Phillips et al., 2017).) Therefore, 429 although our biophysical experiments demonstrate that intra-subtype cis interactions are 430 comparatively weak and, in some cases undetectable in solution, intra-subtype cis dimers likely 431 assemble when constrained in more native membrane environments. As such, while $\alpha$ cPcdhs and 
$432 \gamma \mathrm{C} 4$ are obligate participants in cis-heterodimers, at least in their cell surface transport, our data

433 show that the remaining cPcdhs are preferentially, although not exclusively, participants in cis-

434 heterodimers.

The cis binding preferences indicated by our data can be largely understood in terms of the

437 asymmetric interface discussed above. Specifically, different isoforms preferentially form one side

438 of the cis dimer: for example, the EC6-only side for cPcdh- $\gamma \mathrm{A} 4$ and the EC5-6 side for cPcdh-

$439 \gamma \mathrm{C} 3$. Homodimerization requires participation of single isoform on both sides of an interface

440 posing challenges in the optimization of binding affinities since, in some cases, the same residue

441 must participate in different intermolecular interactions. Given significant sequence conservation

442 in all members of an alternate cPcdh subfamily (Figure 4-figure supplement 3) even intra-

443 subfamily heterophilic interactions are more difficult to optimize relative to inter-subfamily

444 heterodimerization where there are no constraints on the two interacting surfaces. Additionally,

445 the robust cell surface delivery of many cPcdhs in cells expressing only a single isoform also

446 suggests that all carrier isoforms - $\beta$-, $\gamma \mathrm{A}$-, and $\gamma \mathrm{B}$-cPcdhs, plus $\mathrm{C}$-types $\alpha \mathrm{C} 2, \gamma \mathrm{C} 3$, and $\gamma \mathrm{C} 5-\mathrm{can}$

447 fill both the EC6 and EC5-6 roles, as cis-dimer formation is thought to be required for cell surface

448 export (Goodman et al., 2017; Goodman et al., 2016b; Thu et al., 2014). Therefore side preferences

449 are most likely not absolute for carrier $\mathrm{cPcdh}$ isoforms and may vary among individual isoform

450 and/or subtype pairings.

452 Functional implications of $c P c d h$ interactions - The functional role of precise trans homophilic 453 specificity in ensuring high fidelity discrimination between neuron self and non-self has been 454 discussed previously (Rubinstein et al., 2017; Rubinstein et al., 2015) and is summarized above. It 455 is an essential feature of the chain termination model. The role of promiscuous cis interactions can 456 also be understood in terms of this model in that cis promiscuity enables the formation of a large 457 and diverse set of cis dimers that can only form long molecular zippers when all isoforms are 458 matched. However, the results of this study reveal strong preferences for inter-subgroup 459 heterophilic interactions whose biological rationale is uncertain. cPcdhs from the three subfamilies 460 have been shown to act cooperatively in certain neuronal contexts although whether this relates to 461 their cis interactions is unknown (Hasegawa et al., 2016; Ing-Esteves et al., 2018). 
463 One possible advantage of weak homophilic cis interactions would be to ensure that once reaching 464 the cell surface a diverse set of cis dimers forms. This explanation implicitly assumes that most 465 isoforms (except for $\alpha$-Pcdhs and $\gamma \mathrm{C} 4$ ) reach the surface as homodimers that must then quickly 466 dissociate and form more stable heterodimers. Another explanation posits that homotypic zippers 467 consisting solely of cis-homodimers are kinetically easier to form than heterotypic zippers since 468 in a homotypic zipper, either "wing" of the new cis dimer can form trans interactions with the 469 wing at the chain terminus. In contrast, in a hetero-dimeric zipper, only one wing can form 470 homophilic interactions with the chain terminus (Figure 1D). A preference for homotypic zippers 471 would then reduce the diversity required in the chain termination model since, in this model, it is 472 essential that all isoforms be incorporated into a growing zipper. The formation of long homotypic 473 zippers might lead to a repulsive phenotype even when mismatches are present.

However, these explanations would not fully account for interfamily heterophilic preferences. One possibility is suggested by the observation that C-types are often highly expressed compared to alternate cPcdhs, for example in Purkinje cells (Esumi et al., 2005; Kaneko et al., 2006). To ensure sufficient diversity in growing zippers, it would then be important to ensure that zippers that are formed are not overly enriched in C-type isoforms as would be accomplished through preferential heterophilic cis interactions. This same logic would also pertain to alternate $\mathrm{cPcdh}$ in cases where one subfamily is more heavily expressed than another.

483 C-type cPcdhs have different functions than alternate cPcdhs and these are reflected in different 484 expression patterns. For example, $\alpha \mathrm{C} 2$ can be alone responsible for tiling (Chen et al., 2017) (Of 485 note, in the chain termination model, a completely homophilic zipper is sufficient to initiate self486 avoidance facilitating tiling). On the other hand $\gamma \mathrm{C} 4$, which has a unique and crucial role in 487 neuronal survival (Garrett et al., 2019), requires co-expression with another cPcdh isoform for 488 robust cell surface expression and therefore is likely unable to act in isolation (Thu et al., 2014). 489 Furthermore, as detailed above, $\gamma \mathrm{C} 4$ has a much weaker trans interaction affinity than any other $490 \mathrm{cPcdh}$ isoform measured to date, although it is still able to mediate cell aggregation when delivered 491 to the cell surface (Thu et al., 2014). The presence of E78 appears in large part to be responsible 492 for this weak affinity. It is unclear whether $\gamma \mathrm{C} 4$ 's weak trans affinity plays any functional role, 493 although a weak homodimer interaction may facilitate extracellular interactions with other, 
494 currently unidentified, proteins. More generally, it seems likely that different intracellular 495 interactions account for the specialized functions of C-type Pcdhs. The cytoplasmic domain plays 496 an important role in the activation of Wnt, WAVE, and other signaling cascades (Chen et al., 2009;

497 Fukuda et al., 2008; Keeler et al., 2015; Mah and Weiner, 2017; Onouchi et al., 2015; Pancho et 498 al., 2020). In some cases, the cytoplasmic domains of a subset or even a single cPcdh isoform 499 activates a specific signaling cascade. For example, $\mathrm{cPcdh} \gamma \mathrm{C} 3$ is the only isoform able to interact 500 and inhibit Axin1, a Wnt pathway activator (Mah et al., 2016). Of note, $\gamma$-cPcdh intracellular 501 domains consist of a C-terminal constant region common to all $\gamma$ isoforms (including the three $\gamma$ 502 C-types) and a membrane-proximal variable region consisting of $\sim 100$ residues that could account 503 for the unique intracellular interactions and signaling of individual isoforms. Additionally it is 504 possible that extracellular interactions to molecules from other families, such as Neuroligins, may 505 account for some distinctions in function (Molumby et al., 2017; Steffen et al., 2021).

507 Overall, the results of this study demonstrate the remarkable tuning of the interactions among 508 clustered protocadherin family members: homophilic trans interactions are remarkably specific 509 despite the high level of sequence identity among family members while cis interactions, though 510 somewhat promiscuous, also appear designed to have binding preferences of still uncertain 511 function. These binding properties match requirements of the "isoform-mismatch chain512 termination model" for neuronal self-vs-non-self discrimination in which all expressed cPcdh 513 isoforms assemble into intercellular zippers formed by alternating promiscuous cis and matched 514 trans interactions with assembly size dictated by the presence or absence of mismatched isoforms. 515 It remains to be seen whether such assemblies can be observed in vivo and how they control 516 downstream signaling pathways. 


\section{Materials and Methods}

\section{Protein production and purification}

519 cDNAs for cPcdh ectodomain fragments, excluding the predicted signal sequences, were cloned

520 into a paSHP-H mammalian expression vector (a kind gift from Daniel J. Leahy, John Hopkins

521 University) modified with the human Binding immunoglobulin protein (BiP;

522 MKLSLVAAMLLLLSAARA) signal sequence and a C-terminal octa-histidine tag (Rubinstein et

523 al., 2015). The signal sequences were predicted using the SignalP 4.0 server (Petersen et al., 2011).

524 Point mutations were introduced into cDNA constructs using the KOD hot start polymerase

525 (Novagen) following the standard Quikchange protocol (Stratagene).

527 Suspension-adapted HEK293 Freestyle cells (Invitrogen) in serum free media (Invitrogen) grown

528 and maintained at $37{ }^{\circ} \mathrm{C}$ and $10 \%$ carbon dioxide were used for protein expression. The plasmid 529 constructs were transfected into cells using polyethyleneimine (Polysciences Inc.) (Baldi et al., 530 2012). Media was supplemented with $10 \mathrm{mM} \mathrm{CaCl}_{2} 4$ hours after transfection. Conditioned media 531 was harvested $\sim 6$ days after transfection and the secreted proteins were purified using batch nickel532 nitrilotriacetic acid (Ni-NTA) affinity chromatography followed by size exclusion 533 chromatography over Superdex 200 26/60 column (Cytiva) on an AKTA pure fast protein liquid 534 chromatography system (Cytiva). Purified proteins were concentrated to $>2 \mathrm{mg} / \mathrm{ml}$ in $10 \mathrm{mM}$ Tris$535 \mathrm{Cl} \mathrm{pH} \mathrm{8.0,150} \mathrm{mM} \mathrm{NaCl,} 3 \mathrm{mM} \mathrm{CaCl}_{2}$, and 100-250 mM imidazole $\mathrm{pH} 8.0$ and stored at $4{ }^{\circ} \mathrm{C}$ for 536 short-term use or flash frozen in liquid nitrogen for long-term storage at $-80{ }^{\circ} \mathrm{C}$.

538 Constructs encoding biotinylated cPcdh fragments for immobilization in SPR experiments were 539 prepared by insertion of an Avi-tag (GLNDIFEAQKIEWHE)-encoding sequence between the 540 octa-histidine tag and stop codon. These were co-transfected with a plasmid encoding the biotin541 Ligase BirA from E. coli (Lys2-Lys321) with a BiP signal sequence and a C-terminal endoplasmic 542 reticulum-retention signal (DYKDEL) (Barat and $\mathrm{Wu}, 2007)$. The expression and BirA plasmids 543 were mixed at a 9:1 ratio for transfection and $50 \mu \mathrm{M}$ Biotin (Sigma) was added to the media $4 \mathrm{~h}$ 544 post-transfection. Purification was carried out exactly as for the non-biotinylated constructs and 545 biotinylation was confirmed by western blot using NeutrAvidin-HRP (ThermoFisher). 


\section{Sedimentation equilibrium analytical ultracentrifugation (AUC)}

\begin{tabular}{|c|c|c|}
\hline Protein & $\begin{array}{c}\text { Imidazole } \\
\text { pH } 8.0(\mathrm{mM})\end{array}$ & Spin speeds (rpm) \\
\hline$\alpha 4$ EC1-5 & 100 & $9000,11000,13000,15000$ \\
\hline$\alpha 7$ EC1-5 L301R & 100 & $9000,11000,13000,15000$ \\
\hline$\alpha 12$ EC1-5 (poorly behaved) & 200 & $11000,14000,17000,20000$ \\
\hline$\gamma B 4$ EC1-5 & 200 & $11000,14000,17000,20000$ \\
\hline$\gamma \mathrm{B} 5 \mathrm{EC} 1-4-\mathrm{AVI}$ & 200 & $11000,14000,17000,20000$ \\
\hline$\gamma \mathrm{C} 5 \mathrm{EC} 1-5 \mathrm{~S} 116 \mathrm{R}$ & 200 & $11000,14000,17000,20000$ \\
\hline$\beta 6$ EC1-4 & 100 & $9000,11000,13000,15000$ \\
\hline$\beta 6$ EC1-4-AVI tag & 200 & $11000,14000,17000,20000$ \\
\hline$\beta 6$ EC1-4 R41N & 200 & $11000,14000,17000,20000$ \\
\hline$\beta 6$ EC1-4 S117I & 200 & $11000,14000,17000,20000$ \\
\hline$\beta 6$ EC1-4 L125P & 200 & $11000,14000,17000,20000$ \\
\hline$\beta 6$ EC1-4 E369K & 200 & $11000,14000,17000,20000$ \\
\hline$\beta 6$ EC1-4 Y371F & 200 & $11000,14000,17000,20000$ \\
\hline$\beta 6$ EC1-4 R41N/S117I (precipitates) & 200 & $11000,14000,17000,20000$ \\
\hline$\beta 6$ EC1-4 R41N/E369K & 200 & $11000,14000,17000,20000$ \\
\hline$\beta 6$ EC1-4 S117I/L125P & 200 & $11000,14000,17000,20000$ \\
\hline$\beta 6$ EC1-4 R41N/S117I/L125P & 200 & $11000,14000,17000,20000$ \\
\hline$\beta 6$ EC1-4 R41N/S117I/E369K & 200 & $11000,14000,17000,20000$ \\
\hline$\beta 6$ EC1-4 R41N/E369K/Y371F & 200 & $11000,14000,17000,20000$ \\
\hline$\beta 6$ EC1-4 R41N/S117I/L125P/ E369K/Y371F & 200 & $11000,14000,17000,20000$ \\
\hline$\beta 1$ EC3-6 & 200 & $12000,16000,20000,24000$ \\
\hline$\beta 6$ EC1-6 & 250 & $9000,11000,13000,15000$ \\
\hline$\beta 9$ EC3-6 & 200 & $11000,14000,17000,20000$ \\
\hline$\gamma$ A3 EC3-6 & 200 & $11000,14000,17000,20000$ \\
\hline$\gamma$ A9 EC3-6 & 200 & $11000,14000,17000,20000$ \\
\hline$\gamma \mathrm{B} 7 \mathrm{EC} 3-6$ A570R & 200 & $13000,17000,21000,25000$ \\
\hline$\alpha C 2$ EC3-6-AVI tag & 200 & $11000,14000,17000,20000$ \\
\hline$\gamma \mathrm{C5}$ EC2-6 & 250 & $9000,11000,13000,15000$ \\
\hline$\gamma \mathrm{C} 4 \mathrm{EC} 1-4$ & 250 & $11000,14000,17000,20000$ \\
\hline$\gamma C 4$ EC1-4 D290A & 250 & $11000,14000,17000,20000$ \\
\hline$\gamma \mathrm{C} 4 \mathrm{EC} 1-4 \mathrm{D} 290 \mathrm{~N}$ & 250 & $11000,14000,17000,20000$ \\
\hline$\gamma \mathrm{C} 4 \mathrm{EC} 1-4 \mathrm{E} 78 \mathrm{~A}$ & 250 & $11000,14000,17000,20000$ \\
\hline$\gamma \mathrm{C} 4 \mathrm{EC} 1-4 \mathrm{E} 78 \mathrm{Q}$ & 250 & $11000,14000,17000,20000$ \\
\hline$\gamma \mathrm{C} 4 \mathrm{EC} 1-4 \mathrm{~S} 344 \mathrm{R}$ & 250 & $11000,14000,17000,20000$ \\
\hline
\end{tabular}

548 Experiments were performed in a Beckman XL-A/I analytical ultracentrifuge (Beckman-Coulter,

549 Palo Alto CA, USA), utilizing six-cell centerpieces with straight walls, $12 \mathrm{~mm}$ path length and 550 sapphire windows. Protein samples were dialyzed overnight and then diluted in $10 \mathrm{mM}$ Tris-Cl

$551 \mathrm{pH} 8.0,150 \mathrm{mM} \mathrm{NaCl}, 3 \mathrm{mM} \mathrm{CaCl}_{2}$ with $100-250 \mathrm{mM}$ imidazole $\mathrm{pH} 8.0$, as detailed in the above

552 table. The samples were diluted to an absorbance of $0.65,0.43$ and 0.23 at $10 \mathrm{~mm}$ and $280 \mathrm{~nm}$ in

553 channels A, B and C, respectively. For each sample, buffer was used as blank. The samples were

554 run in duplicate at four speeds as detailed in the above table. The lowest speed was held for $20 \mathrm{~h}$ 
then four scans were conducted with $1 \mathrm{~h}$ interval, the subsequent three speeds were each held for

$55610 \mathrm{~h}$ followed by four scans with 1 hour interval each. Measurements were taken at $25^{\circ} \mathrm{C}$, and

557 detection was by UV at $280 \mathrm{~nm}$ or interference. Solvent density and protein v-bar at both

558 temperatures were determined using the program SednTerp (Alliance Protein Laboratories, Corte

559 Cancion, Thousand Oaks, CA, USA). The molecular weight of each protomer used in AUC

560 experiments, was determined by MALDI mass spectrometry. For calculation of dimeric $\mathrm{K}_{\mathrm{D}}$ and

561 apparent molecular weight, all data were used in a global fit, using the program HeteroAnalysis,

562 (www.biotech.uconn.edu/auf). Calculation of the tetramer $\mathrm{K}_{\mathrm{d}} \mathrm{S}$ was done with the program Sedphat

563 (http://www.analyticalultracentrifugation.com/sedphat/index.htm).

565 Surface plasmon resonance (SPR) binding experiments

566 SPR binding experiments were performed using a Biacore T100 biosensor equipped with a Series

567 S CM4 sensor chip, immobilized with NeutrAvidin over all four flow cells. NeutrAvidin 568 immobilization was performed in HBS-P buffer, $\mathrm{pH} 7.4$ at $32{ }^{\circ} \mathrm{C}$, over all four surfaces using 569 amine-coupling chemistry as described in Katsamba et al. (2009), resulting in approximately 570 10,000 RU of NeutrAvidin immobilized (Katsamba et al., 2009). Binding experiments were 571 performed at $25{ }^{\circ} \mathrm{C}$ in a running buffer containing $10 \mathrm{mM}$ Tris-Cl pH 8.0, $150 \mathrm{mM} \mathrm{NaCl}, 3 \mathrm{mM}$ $572 \mathrm{CaCl}_{2}, 20 \mathrm{mM}$ imidazole, $0.25 \mathrm{mg} / \mathrm{mL} \mathrm{BSA}$ and $0.005 \%$ (v/v) Tween-20 unless otherwise noted.

574 C-terminal biotinylated fragments were tethered over individual NeutrAvidin-immobilized flow 575 cells (shown in the left column of each Figures 2, 4, 5C, Figure 2-figure supplement 1, Figure 5762 -figure supplement 2B, Figure 4-figure supplement 1 and Figure 5-figure supplement 2B) at 577 2300-3000 RU, depending on the experiment, using a flow rate of $20 \mu \mathrm{L} / \mathrm{min}$. A NeutrAvidin578 immobilized flow cell was used as a reference in each experiment to subtract bulk refractive index 579 changes. The analytes tested in each experiment are listed at the top row. All analytes (with 580 exceptions for the $c$ is interacting pairs $\gamma \mathrm{C}_{3-6} / \beta 9_{3-6}$, in both orientations, and $\beta 6_{1-6} / \gamma \mathrm{C} 3_{3-6}$ in Figure 5814 A, discussed below) were tested at six concentrations ranging between 24, 8, 2.667, 0.889, 0.296, 582 and $0.099 \mu \mathrm{M}$, prepared using a three-fold dilution series. $\gamma \mathrm{C} 3_{3-6}$ binding over $\beta 9_{3-6}$ (Figure 4A) 583 was tested at five concentrations from $8-0.099 \mu \mathrm{M}$. 
584 For all experiments, analyte samples were injected over the captured surfaces at $50 \mu \mathrm{L} / \mathrm{min}$ for 40

$585 \mathrm{~s}$, followed by $180 \mathrm{~s}$ of dissociation phase, a running buffer wash step and a buffer injection at 100

$586 \mu \mathrm{L} / \mathrm{min}$ for $60 \mathrm{~s}$. Protein samples were tested in order of increasing concentration, and within the

587 same experiment the entire concentration series was repeated to confirm reproducibility. Every

588 three binding cycles, buffer was used as an analyte instead of a protein sample to double reference

589 the binding responses by removing systematic noise and instrument drift. The resulting binding

590 curves were normalized for molecular weight differences according to data provided by mass spec

591 for each molecule. The data was processed using Scrubber 2.0 (BioLogic Software). To provide

592 an estimate of the number of possible heterophilic binding pairs, we have used a cut-off of 40RU,

593 which is the lowest signal that can be observed for a homodimeric cis fragment pair, $\gamma \mathrm{B} 2{ }_{3-6}$.

In Figure 4A, $\beta 6_{1-6}$ and $\beta 9_{3-6}$ were tested over $\gamma \mathrm{C} 3_{3-6}$ at six concentrations ranging from 900 to 3.7 $\mathrm{nM}$, which is 27 -fold lower than the other interactions, prepared using a three-fold dilution series in a running buffer containing increased concentrations of imidazole $(100 \mathrm{mM})$ and BSA $(0.5$ $\mathrm{mg} / \mathrm{mL}$ ) to minimize nonspecific interactions. For these two interactions, although analyte samples were injected over the captured surfaces at $50 \mu \mathrm{L} / \mathrm{min}$ for $40 \mathrm{~s}$, the dissociation phase was monitored for 300 s to provide additional time for complex dissociation. Nevertheless, higher analyte concentrations produced binding profiles that were not reproducible, most likely due to the fact that bound complexes could not dissociate completely at these higher concentrations.

For the calculation of heterophilic $\mathrm{K}_{\mathrm{DS}}$ for the monomeric cis fragments $\beta 1_{3-6}, \gamma \mathrm{A}_{3_{-6},}, \gamma \mathrm{A}_{3_{-6}}$ and $\gamma \mathrm{C} 3_{3-6}$ over each of the six surfaces, except $\beta 9_{3-6}$, the duplicate binding responses were fit globally, using an 1:1 interaction model and a single $\mathrm{K}_{\mathrm{D}}$ was calculated as the analyte concentration that would yield $0.5 \mathrm{R}_{\max }$ and a fitting error, indicated in brackets. $\mathrm{K}_{\mathrm{DS}}$ lower than $24 \mu \mathrm{M}$ were calculated using an independent $R_{\max }$. For $K_{D S}$ greater $24 \mu \mathrm{M}$, the $\mathrm{R}_{\max }$ was fixed to a global value determined by the $\mathrm{R}_{\max }$ of a different cPcdh analyte tested over the same surface during the same

610 experiment that showed binding above $50 \%$ and therefore produced a more accurate $\mathrm{R}_{\max }$. For $\mathrm{K}_{\mathrm{D} S}$

$611>50 \mu \mathrm{M}$, a lower limit is listed since at the analyte concentrations used, $(0.098-24 \mu \mathrm{M})$, accurate

$612 \mathrm{~K}_{\mathrm{DS}}$ could not be determined, even when the $\mathrm{R}_{\max }$ is fixed. NB (No Binding) represents 613 interactions that did not yield any binding signal. The binding curves of $\gamma \mathrm{C} 3_{3-6}$ over the $\beta 9_{3-6}$ did 614 not come to equilibrium during the time-course of the experiment, so a kinetic analysis was 
615 performed to calculate a $\mathrm{K}_{\mathrm{D}}$ (Figure 4 - figure supplement $1 \mathrm{~A}$ ). Binding of $\gamma \mathrm{C} 3_{3-6}$ was tested using

616 a concentration range of $900-0.411 \mathrm{nM}$ prepared using a three-fold dilution series in a running

617 buffer containing increased concentrations or imidazole $(100 \mathrm{mM})$ and BSA $(0.5 \mathrm{mg} / \mathrm{mL})$ to

618 minimize any nonspecific interactions. Protein samples were injected over the captured surfaces

619 at $50 \mu \mathrm{L} / \mathrm{min}$ for $90 \mathrm{~s}$, followed by $420 \mathrm{~s}$ of dissociation phase, a running buffer wash step and a

620 buffer injection at $100 \mu \mathrm{L} / \mathrm{min}$ for $60 \mathrm{~s}$. Protein samples were tested in order of increasing

621 concentration in triplicate to confirm reproducibility. Every three binding cycles, buffer was used

622 as an analyte instead of a protein sample to double reference the binding responses by removing 623 systematic noise and instrument drift. The binding data was analyzed using an 1:1 interaction 624 model to calculate the kinetic parameters and the $\mathrm{K}_{\mathrm{D}}$.

K562 cell aggregation assays

627 Full-length cPcdhs $\beta 6$ and $\beta 8$ cDNAs were cloned into the pMax expression vectors encoding C628 terminal mCherry or mVenus tagged cPcdh proteins, then transfected into K562 cells (ATCC 629 CCL243) as previously described (Goodman et al., 2017; Thu et al., 2014). Point mutants were 630 generated using the QuikChange method (Stratagene). In brief, K562 cells were cultured at $37^{\circ} \mathrm{C}$ 631 with 5\% $\mathrm{CO}_{2}$ in DMEM with GlutaMAX (GIBCO) supplemented with 10\% FBS and 1\% 632 penicillin-streptomycin for two days. Next, cells were counted, centrifuged, and resuspended at a 633 density of $\sim 1.5 \times 10^{4}$ cells $/ \mu \mathrm{L}$ in SF Cell Line 4D-Nucleofector Solution SF with supplement 634 according to manufacturer instructions (Lonza). $2 \mu \mathrm{g}$ of each Pcdh expression construct were 635 transfected into $20 \mu \mathrm{L}$ of the K562 cell suspension by electroporation using an Amaxa 4D636 Nucleofector (Lonza). Transfected cells were transferred to a 24 -well plate in $500 \mu \mathrm{L}$ of medium 637 per well and incubated overnight at $37^{\circ} \mathrm{C}$ and $5 \% \mathrm{CO}_{2}$. Cells then were mixed, re-incubated with 638 gentle rocking for 4 hours, then imaged with an Olympus IX73 fluorescent microscope to 639 determine the extent of aggregation.

\section{Size-exclusion coupled multi-angle light scattering (SEC-MALS)}

642 SEC-MALS experiments were performed using a Superdex 200 Increase 3.2/300 size exclusion 643 column on an AKTA FPLC system (Cytiva) coupled to inline static light scattering (Dawn Heleos 644 II, Wyatt Technology), differential refractive index (Optilab rEX, Wyatt Technology) and UV 645 detection. Purified cPcdh proteins were diluted to $18 \mu \mathrm{M}$ in running buffer $(150 \mathrm{mM} \mathrm{NaCl}, 10 \mathrm{mM}$ 
646 Tris- $\mathrm{Cl} \mathrm{pH} \mathrm{8,} 3 \mathrm{mM} \mathrm{CaCl}_{2}, 200 \mathrm{mM}$ Imidazole $\mathrm{pH}$ 8) and 50 or $100 \mu 1$ samples were run at a flow

647 rate of $0.5 \mathrm{ml} / \mathrm{min}$ at room temperature. Mixtures of cPcdh fragments were prepared in the same 648 buffer at final concentrations of $18 \mu \mathrm{M}$ for each protein and run under the same conditions. Data 649 were analyzed using ASTRA software (Wyatt Technologies).

During SEC-MALS experiments, a dimer/monomer equilibrium is established as proteins move 652 through the size exclusion chromatography column, which is influenced by the $\mathrm{K}_{\mathrm{D}}$ of the 653 interaction. The concentrations used in the current experiments (18 $\mu \mathrm{M}$ for each cPcdh fragment), 654 although above the $\mathrm{K}_{\mathrm{D}}$ of $3 \mu \mathrm{M}$ for the $\gamma \mathrm{C} 3 / \gamma \mathrm{A} 4$ cis interaction, are not sufficiently high for all the 655 cis fragments to be bound into heterodimers, leaving a significant population of molecules as 656 monomers, resulting in apparent molecular weights of $\sim 76 \mathrm{kDa}$ for the dimeric species compared 657 to the predicted molecular weight for a dimer of $\sim 108 \mathrm{kDa}$. For the $\gamma \mathrm{A} 4+\gamma \mathrm{C} 3 \mathrm{~V} 560 \mathrm{R}$ mixture, a 658 lower $\mathrm{K}_{\mathrm{D}}$ allows for only a small proportion of molecules to assemble into heterodimers at $18 \mu \mathrm{M}$, 659 therefore the dimer and monomer peaks elute at different volumes and are completely resolved.

661 X-ray crystallography

662 Crystallization screening of $\gamma \mathrm{C}_{1-4}$ using the vapor diffusion method yielded two protein crystal 663 forms: The first crystal form crystals were grown using a protein concentration of $7 \mathrm{mg} / \mathrm{ml}$ in $10 \%$ 664 (w/v) PEG8000, 20\% ethylene glycol, 10\% Morpheus Amino Acids (Molecular Dimensions), and 6650.1 M Morpheus Buffer System 2 (Hepes/MOPS buffer; Molecular Dimensions) pH 7.5. No 666 additional cryoprotection was required for this crystal form. The second crystal form crystals were 667 grown using a protein concentration of $7 \mathrm{mg} / \mathrm{ml}$ in $1 \mathrm{M} \mathrm{LiCl}, 0.1 \mathrm{M} \mathrm{Mes} \mathrm{pH} \mathrm{6.0,} \mathrm{and} \mathrm{10 \%} \mathrm{(w/v)}$ 668 PEG6000. The crystal used for data collection was cryo-protected in the crystallization condition 669 plus 30\% (w/v) glycerol. X-ray diffraction data for each crystal form were collected at $100 \mathrm{~K}$ from 670 single crystals at Northeastern Collaborative Access Team (NE-CAT) beamline 24ID-E at the 671 Advanced Photon Source, Argonne National Laboratory.

$673 \gamma_{C 4-4}$ crystal form 1: Diffraction anisotropy and pseudosymmetry

674 The X-ray diffraction data for the first crystal form showed strong diffraction anisotropy, with 675 relatively strong diffraction along $\mathrm{c}^{*}$ and much weaker diffraction along $\mathrm{a}^{*}$ and $\mathrm{b}^{*}$ (Figure 3676 figure supplement 1A). These data were therefore truncated using ellipsoidal limits with using a 
$6773.0 \mathrm{~F} /$ sigma cut-off along each of the three principal crystal axes as implemented in the UCLA

678 Diffraction Anisotropy Server (Strong et al., 2006) to 4.6/3.9/3.5 $\AA$. The completeness within the 679 applied ellipsoidal resolution limits was 96.8\% (Figure 3-source data 1).

$\chi_{C} 4_{1-4}$ crystal form 1: Crystal structure phasing and refinement

682 The $\gamma \mathrm{C}_{1-4}$ crystal structure was solved by molecular replacement using Phaser (McCoy et al., 683 2007), implemented in CCP4 (Winn et al., 2011). The $\gamma \mathrm{C} 5_{\mathrm{EC} 1-3}$ crystal structure (PDB: 4ZPO) 684 modified using a sequence alignment to $\gamma \mathrm{C} 4$ with Phenix's MRage program (Liebschner et al., 685 2019) was used as a search model. Following an initial round of rigid body refinement in Phenix 686 (Liebschner et al., 2019) the EC domain 4 from the $\alpha 7_{\mathrm{EC} 1-5}$ crystal structure (PDB: 5DZV) was manually placed into the electron density map, using structural alignment to the EC1-3 regions as

688 a guide. The resulting model was subjected to a further round of rigid body refinement. At this 689 stage there was clear difference density for the interdomain calcium ions and covalently linked 690 glycans not present in the models. Iterative model building using Coot (Emsley et al., 2010) and 691 maximum-likelihood refinement using Phenix (Liebschner et al., 2019) was subsequently 692 conducted. The higher resolution (2.4 $\AA$ ) crystal form 2 crystal structure (see below) was used as 693 a reference model in later rounds of iterative model-building and refinement to guide the local 694 geometry choices in this lower resolution structure. Final refinement statistics are given in Figure 6953 - source data 1.

$\gamma^{C} 4_{1-4}$ crystal form 2: data processing, phasing, and refinement

698 The $\gamma \mathrm{C}_{1-4}$ crystal form 2 dataset was indexed using XDS (Kabsch, 2010) and scaled using 699 AIMLESS (Evans and Murshudov, 2013). The data was spherically truncated with high resolution 700 limit of $2.4 \AA$. Data collection statistics are given in Figure 3-source data 1.

702 The $\gamma \mathrm{C}_{1-4}$ crystal form 2 crystal structure has two molecules in the asymmetric unit was solved 703 by molecular replacement using Phaser (McCoy et al., 2007), implemented in Phenix (Liebschner 704 et al., 2019), using the EC2-3 portion of the trans-dimer from the crystal form 1 crystal structure 705 early in refinement as a search model. The molecular replacement solution was then subjected to 706 an initial round of rigid body refinement using Phenix, followed by two rounds of model building 
707 in Coot (Emsley et al., 2010) and maximum likelihood refinement in Phenix. The two EC4

708 domains were then manually placed in the electron density and subjected to rigid body refinement.

709 Following a further two iterative rounds of model building and refinement the two EC1 domains

710 were manually placed. Iterative model-building and refinement continued yielding the final crystal

711 structure whose statistics are given in Figure 3-source data 1.

712

\section{Structure analysis}

714 Buried surface areas were calculated using 'Protein interfaces, surfaces and assemblies' service

715 (PISA) at the European Bioinformatics Institute (http://www.ebi.ac.uk/pdbe/prot int/pistart.html)

716 (Krissinel and Henrick, 2007) and are given as the change in accessible surface area over both

717 protomers. Root mean square deviations over aligned $\mathrm{C} \alpha$ atoms (RMSDs) between structures were

718 calculated using Pymol (Schrödinger, LLC). Crystal structure figures were made using Pymol

719 (Schrödinger, LLC).

\section{Sequence analysis}

722 Multiple sequence alignments were generated using Clustal Omega (Sievers et al., 2011) and 723 visualized using ESPript3.0 (Robert and Gouet, 2014). Sequence logos were generated from 724 multiple sequence alignments using WebLogo3 (Crooks et al., 2004).

\section{Amino acid sequence alignment of cPcdhs $\gamma B 7, \gamma A 4$, and $\gamma C 3$ EC1-6 regions}

CLUSTAL O(1.2.4) multiple sequence alignment 


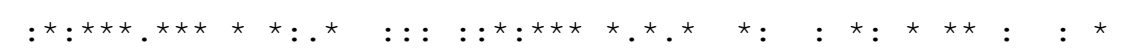

\section{Structure-based sequence analysis of the $\gamma \mathrm{A} 4 / \gamma \mathrm{C} 3$ interaction}

785 Since both $\gamma \mathrm{A}_{4-6}$ and $\gamma \mathrm{C}_{3-6}$ are monomeric in solution but form a robust heterodimer when mixed

786 (in SPR, AUC, and SEC-MALS) we hypothesized that these molecules might have opposing cis

787 interaction side preferences. To facilitate hypothesis generation on the nature of their cis

788 heterodimer interaction we modeled the two possible $\gamma \mathrm{A} 4 / \gamma \mathrm{C} 3$ cis dimers: one with $\gamma \mathrm{A} 4$ occupying

789 the EC6-only position and $\gamma \mathrm{C} 3$ the EC5-6 position; and the second with $\gamma \mathrm{C} 3$ in the EC6-only

790 position and $\gamma \mathrm{A} 4$ in the EC5-6 position. To do this the monomeric $\gamma \mathrm{A} 4_{\mathrm{EC} 3-6}$ crystal structure (PDB:

791 5SZQ) was structurally superimposed over EC6 domains with the EC6-only protomer from the

$792 \gamma \mathrm{B} 77_{\mathrm{EC} 3-6}$ cis-dimer crystal structure (PDB: 5V5X; RMSD 0.7 Å over 91 aligned Cas) or over EC5-

7936 domains with the EC5-6 protomer (RMSD $1.0 \AA$ A over 194 aligned Cas). Since $\gamma$ A4 and $\gamma$ B7 are 
794 so structurally similar in their EC5-6 regions modeling $\gamma$ A4's cis interactions in this manner as a

795 basis for hypothesis generation seemed reasonable. The only region of significant structural

796 deviation within the EC5-6 regions between $\gamma \mathrm{A} 4$ and $\gamma \mathrm{B} 7$ is in the EC6 A-A' loop region which

797 has a peripheral role in the EC6-only protomer interface. For modelling $\gamma \mathrm{C} 3$ we used

798 computational mutagenesis of the $\gamma \mathrm{B} 7$ structure selecting the best-fit rotamer for each amino acid

799 from the Dunbrack rotamer library (Shapovalov and Dunbrack, 2011), implemented in UCSF

800 Chimera (Pettersen et al., 2004). No energy minimization was conducted and the models are

801 intended only for use in hypothesis generation.

802

\section{Cis interface mutants}

804 Our studies of Pcdh cis interactions we have found that mutagenesis of the cis interface commonly 805 has a deleterious impact on protein expression levels in our system (Goodman et al., 2017). We 806 assume this is because cis interaction is required for robust cell-surface delivery/secretion (Thu et 807 al., 2014), although this hasn't been specifically addressed in our HEK293 protein expression 808 system.

810 To test our structure-guided hypotheses regarding $\gamma \mathrm{A} 4$ and $\gamma \mathrm{C} 3 \mathrm{~s}$ ' cis interactions and side 811 preferences as we tried to make a number of different cis interface mutants and were able to obtain 812 four different mutants (see table below). Since protein yields were generally too low for AUC and 813 SPR, MALS was used to study the impact of these mutants on $\gamma \mathrm{A} 4 / \gamma \mathrm{C} 3 \mathrm{cis}$ dimer formation. 


\begin{tabular}{|c|c|c|}
\hline $\begin{array}{l}\text { Mutant protein } \\
\text { ( } \mathrm{B} 7 \text { numbering given in parentheses) }\end{array}$ & $\begin{array}{l}\text { Cis interface side } \\
\text { targeted }\end{array}$ & $\begin{array}{l}\text { Protein expression in } \\
25 \mathrm{~mL} \text { test }\end{array}$ \\
\hline$\gamma$ C3 EC3-6 Y540G (Y532G equivalent) & EC6-only & No \\
\hline rC3 EC3-6 V560D (L555D equivalent) & EC6-only & No \\
\hline rC3 EC3-6 V565R (V560R equivalent) & EC6-only & Yes \\
\hline$\gamma$ C3 EC3-6 A575R (A570R equivalent) & EC5-6 & No \\
\hline rC3 EC3-6 R563K (K558R equivalent) & Both & Yes \\
\hline$\gamma$ A4 EC3-6 Y536G (Y532G equivalent) & EC6-only & No \\
\hline$\gamma$ A4 EC3-6 L559D (L555D equivalent) & EC6-only & No \\
\hline 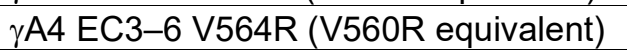 & EC6-only & Yes \\
\hline rA4 EC3-6 A574R (A570R equivalent) & EC5-6 & No \\
\hline rA4 EC3-6 K562R (K558R equivalent) & EC6-only & Yes \\
\hline$\beta 1$ EC3-6 V563R (V560R equivalent) & EC6-only & No \\
\hline$\beta 1$ EC3-6 S573R (A570R equivalent) & EC6-only & No \\
\hline $\beta 1$ EC3-6 K561R (K558R equivalent) & EC5-6 & No \\
\hline$\beta 9$ EC3-6 V563R (V560R equivalent) & EC6-only & No \\
\hline$\beta 9$ EC3-6 A573R (A570R equivalent) & EC6-only & No \\
\hline $\beta 9$ EC3-6 K561R (K558R equivalent) & EC5-6 & No \\
\hline
\end{tabular}




\section{Accession numbers}

817 Atomic coordinates and structure factors for the $\gamma \mathrm{C} 4 \mathrm{EC} 1-4$ crystal structures are deposited in the 818 protein data bank with accession codes PDB: 7JGZ and 7RGF.

\section{Author contributions}

820 K.M.G., B.H., and L.S. designed experiments and analyzed data. S.M., F.B., and K.M.G. cloned,

821 expressed, purified and crystallized the proteins. K.M.G. determined the crystal structures and 822 conducted the sequence and structural analysis. S.M. and F.B. performed the site-directed 823 mutagenesis. P.S.K. performed and analyzed the surface plasmon resonance experiments. G.A. 824 performed and analyzed the analytical ultracentrifugation and multi-angle light scattering 825 experiments. H.D. and R.S. performed and analyzed the cell aggregation experiments. K.M.G. and 826 P.S.K. produced the figures. K.M.G. and L.S. drafted the manuscript. K.M.G., P.S.K., R.R., B.H., 827 and L.S. edited the manuscript.

\section{Acknowledgements}

829 We thank Surajit Banerjee for help with synchrotron data collection at the APS NE-CAT 24-ID$830 \mathrm{C} / \mathrm{E}$ beamlines, supported by NIH P41GM103403. This work was supported by the NIH (grants 831 R01MH1 14817 to L.S. and R01DK106548 to R.S.), the National Science Foundation (grant MCB8321412472 to B.H.), the Israel Science Foundation (grant 1463/19 to R.R.) and the Israel Cancer 833 Research Fund (grant ICRF 19-203-RCDA to R.R.).

\section{Competing Interests}

836 The authors declare no competing interests.

\section{Supplementary Files}

838 Figure 2-figure supplements 1-2 and source data 1

$839 \quad$ Figure 3 -figure supplement 1 and source data 1-2

$840 \quad$ Figure 4-figure supplements 1-3 and source data 1

$841 \quad$ Figure 5-figure supplements 1-2 


\section{References}

843 Baldi, L., Hacker, D.L., Meerschman, C., and Wurm, F.M. (2012). Large-Scale Transfection of Mammalian 844 Cells. In Protein Expression in Mammalian Cells: Methods and Protocols, J.L. Hartley, ed. (Totowa, NJ: 845 Humana Press), pp. 13-26.

846 Barat, B., and Wu, A.M. (2007). Metabolic biotinylation of recombinant antibody by biotin ligase retained in the endoplasmic reticulum. Biomolecular Engineering 24, 283-291.

Bonn, S., Seeburg, P.H., and Schwarz, M.K. (2007). Combinatorial Expression of $\alpha$ - and $\gamma$-Protocadherins Alters Their Presenilin-Dependent Processing. Molecular and Cellular Biology 27, 4121-4132.

Brasch, J., Goodman, K.M., Noble, A.J., Rapp, M., Mannepalli, S., Bahna, F., Dandey, V.P., Bepler, T., Berger, B., Maniatis, T., et al. (2019). Visualization of clustered protocadherin neuronal self-recognition complexes. Nature 569, 280-283.

Brasch, J., Katsamba, P.S., Harrison, O.J., Ahlsén, G., Troyanovsky, R.B., Indra, I., Kaczynska, A., Kaeser, B., Troyanovsky, S., Honig, B., et al. (2018). Homophilic and Heterophilic Interactions of Type II Cadherins Identify Specificity Groups Underlying Cell-Adhesive Behavior. Cell Reports 23, 1840-1852.

Canzio, D., and Maniatis, T. (2019). The generation of a protocadherin cell-surface recognition code for neural circuit assembly. Current Opinion in Neurobiology 59, 213-220.

Chen, J., Lu, Y., Meng, S., Han, M.-H., Lin, C., and Wang, X. (2009). $\alpha$ - and $\gamma$-Protocadherins Negatively

860 Chen, W.V., Nwakeze, C.L., Denny, C.A., O’Keeffe, S., Rieger, M.A., Mountoufaris, G., Kirner, A., 861 Dougherty, J.D., Hen, R., Wu, Q., et al. (2017). Pcdhac2 is required for axonal tiling and assembly of 862 serotonergic circuitries in mice. Science 356, 406-411.

863 Cooper, S.R., Jontes, J.D., and Sotomayor, M. (2016). Structural determinants of adhesion by 864 Protocadherin-19 and implications for its role in epilepsy. eLife 5, e18529.

865 Cosmanescu, F., Katsamba, P.S., Sergeeva, A.P., Ahlsen, G., Patel, S.D., Brewer, J.J., Tan, L., Xu, S., Xiao, 866 Q., Nagarkar-Jaiswal, S., et al. (2018). Neuron-Subtype-Specific Expression, Interaction Affinities, and 867 Specificity Determinants of DIP/Dpr Cell Recognition Proteins. Neuron 100, 1385-1400.e1386.

868 Crooks, G.E., Hon, G., Chandonia, J.-M., and Brenner, S.E. (2004). WebLogo: A Sequence Logo 869 Generator. Genome Research 14, 1188-1190.

870 Emsley, P., Lohkamp, B., Scott, W.G., and Cowtan, K. (2010). Features and development of Coot. Acta 871 Crystallographica Section D: Biological Crystallography 66, 486-501. 
872

Esumi, S., Kakazu, N., Taguchi, Y., Hirayama, T., Sasaki, A., Hirabayashi, T., Koide, T., Kitsukawa, T., Hamada, S., and Yagi, T. (2005). Monoallelic yet combinatorial expression of variable exons of the protocadherin-alpha gene cluster in single neurons. Nature Genetics 37, 171-176.

Evans, P.R., and Murshudov, G.N. (2013). How good are my data and what is the resolution? Acta Crystallographica Section D: Biological Crystallography 69, 1204-1214.

Fan, L., Lu, Y., Shen, X., Shao, H., Suo, L., and Wu, Q. (2018). Alpha protocadherins and Pyk2 kinase regulate cortical neuron migration and cytoskeletal dynamics via Rac1 GTPase and WAVE complex in mice. eLife 7, e35242.

Fukuda, E., Hamada, S., Hasegawa, S., Katori, S., Sanbo, M., Miyakawa, T., Yamamoto, T., Yamamoto, H., Hirabayashi, T., and Yagi, T. (2008). Down-regulation of protocadherin- $\alpha$ A isoforms in mice changes contextual fear conditioning and spatial working memory. European Journal of Neuroscience 28, 13621376.

Garrett, A.M., Bosch, P.J., Steffen, D.M., Fuller, L.C., Marcucci, C.G., Koch, A.A., Bais, P., Weiner, J.A., and Burgess, R.W. (2019). CRISPR/Cas9 interrogation of the mouse Pcdhg gene cluster reveals a crucial isoform-specific role for Pcdhgc4. PLOS Genetics 15, e1008554.

Goodman, K.M., Rubinstein, R., Dan, H., Bahna, F., Mannepalli, S., Ahlsén, G., Aye Thu, C., Sampogna, R.V., Maniatis, T., Honig, B., et al. (2017). Protocadherin cis-dimer architecture and recognition unit diversity. Proceedings of the National Academy of Sciences 114, E9829-E9837.

Goodman, K.M., Rubinstein, R., Thu, C.A., Bahna, F., Mannepalli, S., Ahlsen, G., Rittenhouse, C., Maniatis, T., Honig, B., and Shapiro, L. (2016a). Structural Basis of Diverse Homophilic Recognition by Clustered alpha- and beta-Protocadherins. Neuron 90, 709-723.

Goodman, K.M., Rubinstein, R., Thu, C.A., Mannepalli, S., Bahna, F., Ahlsén, G., Rittenhouse, C., Maniatis, T., Honig, B., and Shapiro, L. (2016b). $\gamma$-Protocadherin structural diversity and functional implications. eLife 5, e20930.

Goodman, K.M., Yamagata, M., Jin, X., Mannepalli, S., Katsamba, P.S., Ahlsén, G., Sergeeva, A.P., Honig, B., Sanes, J.R., and Shapiro, L. (2016c). Molecular basis of sidekick-mediated cell-cell adhesion and specificity. eLife 5, e19058.

Harrison, O.J., Brasch, J., Katsamba, P.S., Ahlsen, G., Noble, A.J., Dan, H., Sampogna, R.V., Potter, C.S., Carragher, B., Honig, B., et al. (2020). Family-wide Structural and Biophysical Analysis of Binding Interactions among Non-clustered $\delta$-Protocadherins. Cell Reports 30, 2655-2671.e2657.

Harrison, O.J., Vendome, J., Brasch, J., Jin, X., Hong, S., Katsamba, P.S., Ahlsen, G., Troyanovsky, R.B., Troyanovsky, S.M., Honig, B., et al. (2012). Nectin ectodomain structures reveal a canonical adhesive interface. Nature Structural \& Molecular Biology 19, 906-915.

Hasegawa, S., Kumagai, M., Hagihara, M., Nishimaru, H., Hirano, K., Kaneko, R., Okayama, A., Hirayama, T., Sanbo, M., and Hirabayashi, M. (2016). Distinct and Cooperative Functions for the 
Protocadherin- $\alpha,-\beta$ and $-\gamma$ Clusters in Neuronal Survival and Axon Targeting. Frontiers in Molecular Neuroscience 9, 155.

Hattori, D., Chen, Y., Matthews, B.J., Salwinski, L., Sabatti, C., Grueber, W.B., and Zipursky, S.L. (2009). Robust discrimination between self and non-self neurites requires thousands of Dscam1 isoforms. Nature $911 \quad 461,644-\mathrm{U} 687$.

Honig, B., and Shapiro, L. (2020). Adhesion Protein Structure, Molecular Affinities, and Principles of Cell-

913 Cell Recognition. Cell 181, 520-535.

914 Hudson, J.D., Tamilselvan, E., Sotomayor, M., and Cooper, S.R. (2021). A complete Protocadherin-19

915 ectodomain model for evaluating epilepsy-causing mutations and potential protein interaction sites.

916 Structure.

917 Ing-Esteves, S., Kostadinov, D., Marocha, J., Sing, A.D., Joseph, K.S., Laboulaye, M.A., Sanes, J.R., and 918 Lefebvre, J.L. (2018). Combinatorial Effects of Alpha- and Gamma-Protocadherins on Neuronal Survival 919 and Dendritic Self-Avoidance. The Journal of Neuroscience 38, 2713-2729. J., Baig, S.M., et al. (2021). Biallelic variants in PCDHGC4 cause a novel neurodevelopmental syndrome with progressive microcephaly, seizures, and joint anomalies. Genetics in Medicine.

Kabsch, W. (2010). XDS. Acta Crystallographica Section D: Biological Crystallography 66, 125-132.

Kaneko, R., Kato, H., Kawamura, Y., Esumi, S., Hirayama, T., Hirabayashi, T., and Yagi, T. (2006). Allelic gene regulation of Pcdh-alpha and Pcdh-gamma clusters involving both monoallelic and biallelic expression in single Purkinje cells. Journal of Biological Chemistry 281, 30551-30560.

Katsamba, P., Carroll, K., Ahlsen, G., Bahna, F., Vendome, J., Posy, S., Rajebhosale, M., Price, S., Jessell, T.M., Ben-Shaul, A., et al. (2009). Linking molecular affinity and cellular specificity in cadherin-mediated adhesion. Proceedings of the National Academy of Sciences 106, 11594-11599.

930 Keeler, A.B., Schreiner, D., and Weiner, J.A. (2015). Protein Kinase C Phosphorylation of a $\gamma$ 931 Protocadherin C-terminal Lipid Binding Domain Regulates Focal Adhesion Kinase Inhibition and Dendrite 932 Arborization. Journal of Biological Chemistry 290, 20674-20686.

933 Kostadinov, D., and Sanes, J.R. (2015). Protocadherin-dependent dendritic self-avoidance regulates neural 934 connectivity and circuit function. Elife 4.

935 Krissinel, E., and Henrick, K. (2007). Inference of Macromolecular Assemblies from Crystalline State. 936 Journal of Molecular Biology 372, 774-797.

937 Lefebvre, J.L., Kostadinov, D., Chen, W.V., Maniatis, T., and Sanes, J.R. (2012). Protocadherins mediate 938 dendritic self-avoidance in the mammalian nervous system. Nature 488, 517-521. 
Liebschner, D., Afonine, P.V., Baker, M.L., Bunkoczi, G., Chen, V.B., Croll, T.I., Hintze, B., Hung, L.W., Jain, S., McCoy, A.J., et al. (2019). Macromolecular structure determination using X-rays, neutrons and electrons: recent developments in Phenix. Acta Crystallographica Section D 75, 861-877.

Mah, K.M., Houston, D.W., and Weiner, J.A. (2016). The gamma-Protocadherin-C3 isoform inhibits canonical Wnt signalling by binding to and stabilizing Axin1 at the membrane. Scientific Reports 6 .

Mah, K.M., and Weiner, J.A. (2017). Regulation of Wnt signaling by protocadherins. Seminars in Cell \& Developmental Biology 69, 158-171.

McCoy, A.J., Grosse-Kunstleve, R.W., Adams, P.D., Winn, M.D., Storoni, L.C., and Read, R.J. (2007). Phaser crystallographic software. Journal of Applied Crystallography 40, 658-674.

Miura, S.K., Martins, A., Zhang, K.X., Graveley, B.R., and Zipursky, S.L. (2013). Probabilistic Splicing of Dscam1 Establishes Identity at the Level of Single Neurons. Cell 155, 1166-1177.

Modak, D., and Sotomayor, M. (2019). Identification of an adhesive interface for the non-clustered $\delta 1$ protocadherin-1 involved in respiratory diseases. Communications Biology 2, 354.

Molumby, M.J., Anderson, R.M., Newbold, D.J., Koblesky, N.K., Garrett, A.M., Schreiner, D., Radley, J.J., and Weiner, J.A. (2017). $\gamma$-Protocadherins Interact with Neuroligin-1 and Negatively Regulate Dendritic Spine Morphogenesis. Cell Reports 18, 2702-2714.

Mountoufaris, G., Canzio, D., Nwakeze, C.L., Chen, W.V., and Maniatis, T. (2016). Writing, Reading, and Translating the Clustered Protocadherin Cell Surface Recognition Code for Neural Circuit Assembly. Annual Review of Cell and Developmental Biology 34, 471-493.

Mountoufaris, G., Chen, W.V., Hirabayashi, Y., O'Keeffe, S., Chevee, M., Nwakeze, C.L., Polleux, F., and Maniatis, T. (2017). Multicluster Pcdh diversity is required for mouse olfactory neural circuit assembly. Science 356, 411-414.

Murata, Y., Hamada, S., Morishita, H., Mutoh, T., and Yagi, T. (2004). Interaction with Protocadherin- $\gamma$ Regulates the Cell Surface Expression of Protocadherin- $\alpha$. Journal of Biological Chemistry 279, 4950849516.

Nicoludis, J.M., Green, A.G., Walujkar, S., May, E.J., Sotomayor, M., Marks, D.S., and Gaudet, R. (2019). Interaction specificity of clustered protocadherins inferred from sequence covariation and structural analysis. Proceedings of the National Academy of Sciences 116, 17825-17830.

Nicoludis, J.M., Lau, S.-Y., Schaerfe, C.P.I., Marks, D.S., Weihofen, W.A., and Gaudet, R. (2015). Structure and Sequence Analyses of Clustered Protocadherins Reveal Antiparallel Interactions that Mediate Homophilic Specificity. Structure 23, 2087-2098.

Nicoludis, J.M., Vogt, B.E., Green, A.G., Scharfe, C.P.I., Marks, D.S., and Gaudet, R. (2016). Antiparallel protocadherin homodimers use distinct affinity- and specificity-mediating regions in cadherin repeats 1-4. eLife 5 . 
973 Onouchi, T., Kishino-Kaneko, Y., Kameshita, I., Ishida, A., and Sueyoshi, N. (2015). Regulation of $974 \mathrm{Ca} 2+/$ calmodulin-dependent protein kinase phosphatase (CaMKP/PPM1F) by protocadherin- $\gamma \mathrm{C} 5$ (Pcdh$975 \gamma \mathrm{C} 5)$. Archives of Biochemistry and Biophysics 585, 109-120.

976 Pancho, A., Aerts, T., Mitsogiannis, M.D., and Seuntjens, E. (2020). Protocadherins at the Crossroad of 977 Signaling Pathways. Front Mol Neurosci 13, 117.

978 Peek, S.L., Mah, K.M., and Weiner, J.A. (2017). Regulation of neural circuit formation by protocadherins. 979 Cellular and Molecular Life Sciences.

980 Petersen, T.N., Brunak, S., von Heijne, G., and Nielsen, H. (2011). SignalP 4.0: discriminating signal peptides from transmembrane regions. Nat Meth 8, 785-786.

982 Pettersen, E.F., Goddard, T.D., Huang, C.C., Couch, G.S., Greenblatt, D.M., Meng, E.C., and Ferrin, T.E. 983 (2004). UCSF Chimera-A visualization system for exploratory research and analysis. Journal of 984 Computational Chemistry 25, 1605-1612. server. Nucleic Acids Research 42, W320-W324.

991 Rubinstein, R., Goodman, K.M., Maniatis, T., Shapiro, L., and Honig, B. (2017). Structural origins of 992 clustered protocadherin-mediated neuronal barcoding. Seminars in Cell \& Developmental Biology 69, 140993150.

994 Rubinstein, R., Thu, C.A., Goodman, K.M., Wolcott, H.N., Bahna, F., Mannepalli, S., Ahlsen, G., Chevee, 995 M., Halim, A., Clausen, H., et al. (2015). Molecular Logic of Neuronal Self-Recognition through 996 Protocadherin Domain Interactions. Cell 163, 629-642.

997 Schmucker, D., Clemens, J.C., Shu, H., Worby, C.A., Xiao, J., Muda, M., Dixon, J.E., and Zipursky, S.L. 998 (2000). Drosophila Dscam is an axon guidance receptor exhibiting extraordinary molecular diversity. Cell 999 101,671-684.

1000 Schreiner, D., and Weiner, J.A. (2010). Combinatorial homophilic interaction between gamma1001 protocadherin multimers greatly expands the molecular diversity of cell adhesion. Proceedings of the 1002 National Academy of Sciences of the United States of America 107, 14893-14898.

1003 Sergeeva, A.P., Katsamba, P.S., Cosmanescu, F., Brewer, J.J., Ahlsen, G., Mannepalli, S., Shapiro, L., and 1004 Honig, B. (2020). DIP/Dpr interactions and the evolutionary design of specificity in protein families. Nat 1005 Commun 11,2125. 
Shapovalov, M.V., and Dunbrack, R.L., Jr. (2011). A smoothed backbone-dependent rotamer library for proteins derived from adaptive kernel density estimates and regressions. Structure 19, 844-858.

Sievers, F., Wilm, A., Dineen, D., Gibson, T.J., Karplus, K., Li, W., Lopez, R., McWilliam, H., Remmert, M., Söding, J., et al. (2011). Fast, scalable generation of high-quality protein multiple sequence alignments using Clustal Omega. Molecular Systems Biology 7, 539-539.

Steffen, D.M., Ferri, S.L., Marcucci, C.G., Blocklinger, K.L., Molumby, M.J., Abel, T., and Weiner, J.A. (2021). The $\gamma$-Protocadherins Interact Physically and Functionally with Neuroligin-2 to Negatively Regulate Inhibitory Synapse Density and Are Required for Normal Social Interaction. Molecular Neurobiology 58, 2574-2589.

Strong, M., Sawaya, M.R., Wang, S., Phillips, M., Cascio, D., and Eisenberg, D. (2006). Toward the structural genomics of complexes: Crystal structure of a PE/PPE protein complex from Mycobacterium tuberculosis. Proceedings of the National Academy of Sciences of the United States of America 103, 80608065 . Interactions between alpha, beta, and gamma Protocadherins. Cell 158, 1045-1059. gene clusters. Genome Research 11,389-404. 
1041 Wu, Y., Honig, B., and Ben-Shaul, A. (2013). Theory and Simulations of Adhesion Receptor Dimerization 1042 on Membrane Surfaces. Biophysical Journal 104, 1221-1229.

1043 Zipursky, S.L., and Grueber, W.B. (2013). The Molecular Basis of Self-Avoidance. In Annual Review of 1044 Neuroscience, Vol 36, S.E. Hyman, ed., pp. 547-568.

1045

1046 
Figure legends:

\section{Figure 1: cPcdh domain organization and extracellular interactions}

1050 (A) Schematic depicting the domain organization of cPcdhs. EC, extracellular cadherin domain;

1051 TM, transmembrane domain; ECD, ectodomain; ICD, intracellular domain.

1052 (B) Schematic of two cPcdhs interacting via the EC1-4 trans interface.

1053 (C) Schematic of two cPcdhs interacting via the EC5-6/EC6 cis interface.

1054 (D) Schematic depiction of the cis/trans cPcdh zipper comprising multiple cPcdh isoforms 1055 (various colors) engaged in homophilic trans interactions and promiscuous cis interactions as 1056 required for the proposed "isoform-mismatch chain-termination model" of cPcdh-mediated 1057 neuronal self-recognition and self-avoidance.

1060 (A) SPR binding profiles of cPcdh trans fragment analytes from all cPcdh subfamilies (denoted in 1061 the top row) flowed over six surfaces coated with alternate cPcdh trans fragments (rows).

1062 Responses over all surfaces are drawn on the same scale and normalized for molecular weight.

1063 (B) SPR binding profiles of cPcdh trans fragment analytes from all cPcdh subfamilies (shown in 1064 columns) flowed over individual surfaces coated with C-type and $\alpha 4 \mathrm{cPcdh}$ trans fragments 1065 (rows). Responses over all surfaces are drawn on the same scale and normalized for molecular 1066 weight.

1068 Figure 3: C-type cPcdh $\gamma \mathrm{C4}$ adopts an EC1-4-mediated head-to-tail trans dimer like 1069 alternate cPcdhs with a comparatively weak dimer affinity

1070 (A) Ribbon diagrams of the $\gamma_{\mathrm{C}} 4_{\mathrm{ECl}-4}$ trans dimer crystal structures obtained from two different 1071 crystal forms. Bound calcium ions are shown as green spheres and glycans are shown in pale blue 1072 spheres.

1073 (B) The two crystal structures have a markedly different trans interface buried surface area (BSA).

1074 Left, Surface views of the two trans dimer crystal structures highlight the difference, with a gap 1075 apparent in the EC2:EC3 region of the interface in crystal form 2 that is absent from crystal form 1076 1. Surfaces are colored by atom type with the carbons colored orange for crystal form 1 and yellow 1077 for crystal form 2. Right, Close up view of the gap region in the crystal form 2 dimer with the side 
1078 chains depicted as sticks. The intact crystal form $1 \gamma \mathrm{C} 4$ dimer is similar overall to those of the

1079 published intact alternate $\alpha, \beta, \gamma \mathrm{A}$, and $\gamma \mathrm{B} \mathrm{cPcdh}$ and the published $\delta 2$ non-clustered (nc) Pcdh

1080 trans dimers (root mean square deviation over aligned Cas (RMSD) 2.4-4.5 A; Figure 3-source

1081 data 2). The published crystal structures of $\gamma \mathrm{A} 8, \gamma \mathrm{A} 1$, and $\gamma \mathrm{B} 3$ also show partially disrupted trans

1082 interfaces though in differing regions of the interface (Goodman et al., 2016b, Nicoludis et al., 1083 2016).

1084 (C) Comparison between the (i) EC1:EC4 and (ii) EC2:EC3 regions of the $\gamma \mathrm{C} 4$ (orange) and $\gamma \mathrm{B} 2$ 1085 (blue, PDB 5T9T) trans dimer interfaces. (i) Structural alignment of the EC1:EC4 portion of the $1086 \gamma \mathrm{C} 4$ and $\gamma \mathrm{B} 2$ trans dimers highlights a possible destabilizing role for $\gamma \mathrm{C} 4$ residue E78 since unlike 1087 its counterpart in $\gamma \mathrm{B} 2$ (D77) it is not juxtaposed with a basic residue. (ii) Similarly, an additional 1088 negatively charged residue (D290) which occupies a central position in the $\gamma \mathrm{C} 4 \mathrm{EC} 2$ :EC3 interface 1089 may also contribute to $\gamma \mathrm{C} 4$ 's comparatively weak trans dimer interaction. Distances between the 1090 D290 side chain and its nearest contacts are shown as dashed grey lines with distances given in 1091 Angstroms.

1092 (D) Sedimentation equilibrium AUC experiments were conducted on $\gamma \mathrm{C} 4 \mathrm{EC} 1-4$ wild type (wt) 1093 and interface mutants to assess whether E78 and D290 negatively impact trans dimerization. Table 1094 details the oligomeric state and dissociation constants for each protein tested.

1095

1096 Figure 4: cPcdh cis interactions are promiscuous with a preference for interfamily 1097 heterodimers

1098 (A) SPR binding profiles of cPcdh cis fragment analytes from all cPcdh subfamilies except alphas 1099 (shown in columns) flowed over individual surfaces coated with cPcdh cis fragments. Binding 1100 profiles for each surface are individually scaled and responses are normalized for molecular 1101 weight.

1102 (B) Table of dissociation constants calculated from the SPR data for the four monomeric analytes. 1103 The number in brackets represents the error of the fit based on analysis of duplicate responses. 1104 Binding signals were not detected for interactions labeled NB (no binding) while $>50$, represents 1105 interactions with $\mathrm{K}_{\mathrm{D}} \mathrm{s}>50 \mu \mathrm{M}$, where an accurate $\mathrm{K}_{\mathrm{D}}$ cannot be determined. 


\section{Figure 5: $\gamma \mathrm{A} 4$ preferentially forms the EC6-only side and $\gamma \mathrm{C3}$ the EC5-6 side in cis dimers}

1109 (A) Structural model of $\gamma \mathrm{A} 4 / \gamma \mathrm{C} 3$ cis dimer based on $\gamma \mathrm{B} 7_{\mathrm{EC} 3-6}$ cis dimer and $\gamma \mathrm{A} 4_{\mathrm{EC} 3-6}$ crystal 1110 structures (PDBs: 5V5X and 5SZQ). $\gamma \mathrm{A} 4$ is shown adopting the EC6-only side (blue protomer) 1111 and $\gamma \mathrm{C} 3$ is shown adopting the EC5-6 side (yellow protomer). Left, schematic of the $\gamma \mathrm{A} 4 / \gamma \mathrm{C} 3$

1112 EC3-6 cis dimer. Right, close-up view of the EC6:EC6 interface from the modeled cis dimer 1113 showing interfacial residue side chains. Bound calcium ions are shown as green spheres. Residues 1114 which were mutated in the panel B are circled in red. $\gamma \mathrm{B} 7$ crystal structure numbering is used for 1115 both $\gamma \mathrm{A} 4$ and $\gamma \mathrm{C} 3$ residues. See methods for $\gamma \mathrm{A} 4$ and $\gamma \mathrm{C} 3$ alignment. Please note the model shown 1116 here is solely for hypothesis generation, since it is unlikely to be completely accurate. See methods 1117 for further details of structural modeling.

1118 (B) Top, SEC-MALS data for an equimolar mixture of wild-type $\gamma \mathrm{A}_{\mathrm{EC}_{-6}-6}$ and $\gamma \mathrm{C} 3_{\mathrm{EC} 3-6}$ showing 1119 dimer formation. Plot shows size exclusion absorbance at $280 \mathrm{~nm}$ trace (left axis), molecular 1120 weight of the eluant peaks (right axis), and the monomer molecular weights of $\gamma \mathrm{A} 4_{\mathrm{EC} 3-6}$ and $1121 \gamma \mathrm{C} 3_{\mathrm{EC} 3-6}$ measured by mass spectrometry $-54.5 \mathrm{kDa}$ and $56.5 \mathrm{kDa}$ respectively - as dashed grey 1122 lines. Average molecular weight of the molecules in the dimer and monomer eluant peaks are 1123 labeled. Middle, SEC-MALS data for V560R mutants, which target the EC6-only side of the 1124 interface. Bottom, SEC-MALS data for residue 558 mutants. The $\gamma$ C3-like K558R mutation in $\gamma$ A4 1125 inhibits heterodimer formation with wild-type $\gamma \mathrm{C} 3$. Similarly, the $\gamma \mathrm{A} 4-$ like R558K in $\gamma \mathrm{C} 3$ inhibits 1126 dimerization with wild-type $\gamma \mathrm{A} 4$.

1127 (C) SPR binding profiles for $\gamma \mathrm{B}_{\mathrm{EC} 3-6}$ wild type and cis interface mutants flowed over three 1128 individual wild-type cis fragment surfaces. The two mutations specifically target one side of the 1129 cis interface. 
1131 Figure 2-figure supplement 1: Trans interface mutants demonstrate homophilic 1132 interactions observed in SPR are mediated by the trans dimer interface

1133 (A) SPR binding curves for wild-type and trans mutant alternate cPcdhs flowed over their 1134 respective immobilized wild-type molecule.

1135 (B) SPR binding curves for wild-type and trans mutant C-type cPcdh $\gamma$ C5 flowed over 1136 immobilized wild-type $\gamma \mathrm{C} 5$.

1138 Figure 2 - figure supplement 2: Mutagenesis experiments reveal role in trans specificity for 1139 the five interfacial residue differences between close pair $\beta 6_{1-4}$ and $\beta 8_{1-4}$

1140 A. Structural superposition of the $\beta 6_{1-4}$ and $\beta 8_{1-4}$ trans dimer crystal structures (PDBs: 5DZX and 1141 5DZY) shown in ribbon depiction above, with close-up views of the trans interfacial regions 1142 containing the five interfacial residues that vary between $\beta 6_{1-4}$ and $\beta 8_{1-4}$ shown below. The two

1143 protomers forming the $\beta 6_{1-4}$ dimer are colored green and pale green respectively. The $\beta 8_{1-4}$ dimer 1144 is colored magenta/light pink. Bound calcium ions are shown as green spheres. Interfacial residue 1145 side chains are shown in the close-up views. The five variable residues are labelled with the $\beta 6_{1-4}$ 1146 amino acid given in green and the $\beta 8_{1-4}$ amino acid in magenta: R/N41 is in EC1; E/K369 and $1147 \mathrm{Y} / \mathrm{F} 371$ are in EC4; S/I117 is in EC2 and self-interacts at the trans dimer center of symmetry; and $1148 \mathrm{~L} / \mathrm{P} 125$ is also in EC2.

1149 B. SPR binding profiles of b6 trans interface mutants converting $\beta 6_{1-4}$ to $\beta 8_{1-4}$ and the wild-type 1150 molecules (shown in columns) were flowed over surfaces coated with wild-type $\beta 6_{1-4}$ or wild-type $1151 \beta 8_{1-4}$ (rows).

1152 C. Results of the K562 co-aggregation assay where cells transfected with mCherry labeled $\beta 6$ and $1153 \beta 8$ wild-types (WT) and the same trans-specificity mutants as in (B) were each mixed with cells 1154 transfected with mVenus labeled $\beta 6$ and $\beta 8$ wild-types (WT). Experiments where the red and green 1155 cells co-aggregate demonstrating interaction between the mCherry-labeled WT or mutant cPcdh 1156 and the mVenus-labeled WT cPcdh are labeled "mixed" and highlighted with magenta boxes. Scale 1157 bar, $100 \mathrm{mM}$. 
1160 Figure 3-figure supplement 1: $\gamma \mathrm{C} 4$ trans dimer crystal structures and trans interface 1161 analysis

1162 (A) Our crystallization experiments with $\gamma \mathrm{C}_{\mathrm{ECl}_{-4}}$ yielded two distinct crystal forms the first of 1163 which showed significant X-ray diffraction anisotropy. (i) UCLA Diffraction Anisotropy Server 1164 (Strong et al., 2006) plot shows the F/sigma by resolution along the $\mathrm{a}^{*}, \mathrm{~b}^{*}$ and $\mathrm{c}^{*}$ axes. (ii) 1165 Synthetic precession photographs of the $\mathrm{X}$-ray diffraction in the $\mathrm{k}=0$ plane (left) and the $\mathrm{h}=0 \mathrm{plane}$ 1166 (right) showing the comparatively stronger/weaker diffraction.

1167 (B) Close up views of the EC1:EC4 and EC2:EC3 interfacial regions from the first crystal form. 1168 One protomer in the symmetric dimer is colored yellow the other orange. Interfacial residues are 1169 labeled, side chains are shown in stick representation and dashed black lines depict potential 1170 interfacial hydrogen bond interactions. The two charged residues, E78 and D290, we selected for 1171 mutagenesis experiments to see whether they play a destabilizing role in the $\gamma \mathrm{C} 4$ trans interaction 1172 are marked with red dashed boxes.

1173 (C) Representative plot of AUC data for the wild type (wt) and mutant $\gamma \mathrm{C} 4 \mathrm{EC} 1-4$ molecules. Raw 1174 data are shown in black circles, and the non-linear fits to a monomer-to-dimer model are shown as 1175 blue lines. The residuals between the data and fits are shown in the plot below. Table detailing the 1176 oligomeric state and dissociation constants determined from the AUC data is shown in Figure 3.

1178 Figure 4-figure supplement 1: Calculation of cis interaction dissociation constants and the 1179 impact of an a-Pedh EC5 on family-wide cis interactions

1180 (A) Kinetic binding analysis of $\gamma \mathrm{C}_{3-6}$ analyte binding over a $\beta 9_{3-6}$ covered surface. Data is shown 1181 in black, and the red traces represent the fit to an 1:1 binding model.

1182 (B) Left, SPR binding profiles from Figure 4 for the four monomeric cis fragment analytes over 1183 all six cis fragment surfaces. Right, fit of the binding data for these four analytes to 1:1 binding 1184 isotherms to calculate $\mathrm{K}_{\mathrm{D}} \mathrm{S} \cdot \gamma \mathrm{A}_{3-6}$ and $\gamma \mathrm{A}_{3-6}$ are monomeric and they are not included in the 1185 binding isotherms over their respective surface.

1186 (C) SPR binding profiles for $\gamma \mathrm{C}_{3-6}$ (from Figure 4) and an $\alpha 7_{1-5} / \gamma \mathrm{C} 3_{6}$ chimera flowed over the 1187 immobilized cis fragment surfaces. Binding profiles for each surface are individually scaled as in 1188 Figure 4. 


\section{Figure 4-figure supplement 2: Range of $\mathrm{cPcdh}$ cis and trans Dissociation constants, $\mathrm{K}_{\mathrm{D}} \mathrm{S}$}

1191 Chart shows the cPcdh trans dimer, homophilic cis dimer, and heterophilic cis dimer interactions

1192 for which we have determined binding affinities divided into four subgroups based on their 1193 dissociation constant. The trans and homophilic cis dimer affinities were determined using AUC

1194 (Figure 2-source data 1 and Figure 4-source data 1) and the heterophilic cis dimer affinities 1195 were determined using SPR (Figure 4B). Of the interactions in the $>50 \mathrm{mM}$ group one trans 1196 interaction and four homophilic cis interactions are monomeric in solution $\left(>500 \mathrm{mM} \mathrm{K}_{\mathrm{D}}\right.$ in 1197 AUC). Three of the 11 heterophilic cis interactions in the $>50 \mathrm{mM}$ group show no binding in our 1198 SPR experiments based on a 40 RU binding threshold.

1200 Figure 4-figure supplement 3: Amino acid sequence alignment reveals conservation of cis 1201 interfacial residues within the alternate $\mathbf{c P c d h}$ subfamilies

1202 (A) Amino acid sequence alignments of cis interfacial residues from the EC6-only and EC5-6 1203 surfaces for all 58 mouse cPcdhs subdivided by subfamily. Completely conserved residues are 1204 highlighted in red with white lettering. Residues 540 and 541 are included in the EC6-only 1205 alignments since the crystal structure of $\gamma$ A4 EC3-6 (PDB: 5SZQ) revealed a distinct EC6 A1206 A'loop architecture to that observed in the $\gamma \mathrm{B} 2,4$, and 7 (PDBs: 5SZR, 6E6B, and 5V5X) cis 1207 fragment crystal structures that would place these residues in the EC6-only interface if maintained 1208 in cis interactions.

1209 (B) Sequence logos based on the sequence alignment shown in (A) for the EC6-only cis interfacial 1210 residues from each of the five $\mathrm{cPcdh}$ subfamilies highlighting the similarities and conserved 1211 differences between the subfamilies. Residues 540 and 541 are included for all isoforms but greyed 1212 out for the non-gA isoforms since their involvement may be gA-specific. NB: Previous studies 1213 have shown that a-Pcdhs have an impaired EC6-only interface (Thu et al., 2014; Goodman et al., 1214 2017).

1215 (C) Sequence logos for the EC5-6 cis interfacial residues from each of the five cPcdh subfamilies. 
1221 Figure 5-figure supplement 1: Structure-guided sequence analysis of $\gamma \mathrm{A} 4$ and $\gamma \mathrm{C3}$ cis interactions

1223 (A) (i) Schematic of the asymmetric $\gamma \mathrm{B} 7_{\mathrm{EC} 3-6}$ cis dimer crystal structure. (ii) Close-up view of the $1224 \gamma \mathrm{B} 7$ cis interface: Interfacial residue side chains are shown in pink for the EC6-only protomer and 1225 purple for the EC5-6 protomer. Bound calcium ions are shown as green spheres.

1226 (B) (i) Schematic of the $\gamma \mathrm{A} 4_{\mathrm{EC} 6} / \gamma \mathrm{C} 3_{\mathrm{EC} 5-6}$ cis dimer. (ii) Model of the $\gamma \mathrm{A} 4_{\mathrm{EC} 6} / \gamma \mathrm{C} 3_{\mathrm{EC} 5-6}$ cis dimer 1227 interaction generated using structural alignment of EC6 from the monomeric $\gamma \mathrm{A} 4$ EC3-6 crystal 1228 structure (PDB 5SZQ) to the $\gamma \mathrm{B} 7 \mathrm{EC} 3-6$ cis dimer structure for the EC6-only side and 1229 computational mutagenesis of $\gamma \mathrm{B} 7$ to $\gamma \mathrm{C} 3$ selecting the best-fit rotamer (without energy 1230 minimization) for the EC5-6 side. The model suggests that this will be the preferred orientation 1231 for the $\gamma \mathrm{A} 4 / \gamma \mathrm{C} 3$ cis dimer interaction. Favorable residue differences between $\gamma \mathrm{B} 7$ from (A) and $1232 \gamma \mathrm{A} 4 / \gamma \mathrm{C} 3$ in this orientation are noted in green. Please note the model shown here is only used for 1233 hypothesis generation, since it is unlikely to be completely accurate.

1234 (C) (i) Schematic of the $\gamma \mathrm{C} 3_{\mathrm{EC} 6} / \gamma \mathrm{A} 4_{\mathrm{EC} 5-6}$ cis dimer. (ii) Model of the $\gamma \mathrm{C} 3_{\mathrm{EC} 6} / \gamma \mathrm{A} 4_{\mathrm{EC} 5-6}$ cis dimer 1235 generated using computational mutagenesis of $\gamma \mathrm{B} 7$ to $\gamma \mathrm{C} 3$ selecting the best-fit rotamer (without 1236 energy minimization) for the EC6-only side and structural alignment of EC5-6 from the $\gamma \mathrm{A} 4$ EC312376 crystal structure to the $\gamma \mathrm{B} 7 \mathrm{EC} 3-6$ cis dimer structure for the EC5-6 side. The model suggests 1238 that this orientation for the $\gamma \mathrm{A} 4 / \gamma \mathrm{C} 3$ cis dimer interaction will be disfavored. Unfavorable residue 1239 differences between $\gamma \mathrm{B} 7$ and $\gamma \mathrm{A} 4 / \gamma \mathrm{C} 3$ in this orientation are noted in red. Please note the model 1240 shown here is unlikely to be completely accurate and is simply for hypothesis generation.

Figure 5-figure supplement 2: $\gamma \mathrm{A} 4$ and $\gamma \mathrm{C3}$ cis-fragments behave as monomers in SEC1243 MALS and mutating $\gamma \mathrm{A} 4$ to make it more like $\gamma \mathrm{C} 3$ prevents $\gamma \mathrm{A} 4 / \gamma \mathrm{C} 3$ cis-heterodimerization (A) SEC-MALS data for wild-type $\gamma \mathrm{A}_{3_{-6}}$, wild-type $\gamma \mathrm{C} 3_{3-6}$, and $\gamma \mathrm{C} 3_{3-6}$ V560R showing all three 1245 molecules are monomeric in SEC-MALS, consistent with their behavior in sedimentation 1246 equilibrium AUC. Plots show size exclusion absorbance at $280 \mathrm{~nm}$ trace in blue (left axis), 1247 molecular weight of the eluant peak in black (right axis), and the monomer molecular weight of $1248 \gamma \mathrm{A}_{3-6}$ or $\gamma \mathrm{C} 3_{3-6}$ measured by mass spectrometry $-54.5 \mathrm{kDa}$ and $56.5 \mathrm{kDa}$ respectively - as dashed 1249 grey lines. Average molecular weight of the molecules in the eluant peaks are labeled. 
1250 (B) SPR binding profiles for $\gamma \mathrm{A}_{3-6}$ wild type and $\gamma \mathrm{A}_{3-6}$ with $\gamma \mathrm{C} 3$-like $c$ is interface mutation 1251 K558R flowed over immobilized wild-type $\gamma \mathrm{C}_{3-6}$. Loss of $\gamma \mathrm{C}_{3-6}$ interaction in the presence of 1252 the K558R mutation is consistent with the SEC-MALS results shown in Figure 5.

1253

1254 


\begin{tabular}{|c|c|c|}
\hline Protein & Oligomeric State & $\begin{array}{c}\text { Dissociation Constant, } \\
K_{D}(\mu M)\end{array}$ \\
\hline \multicolumn{3}{|l|}{ Trans-interacting fragments } \\
\hline$\alpha 4_{1-5}$ & Dimer & $5.0 \pm 0.80$ \\
\hline$\alpha 7^{*}{ }_{1-5}$ & Dimer & $2.91 \pm 0.55$ \\
\hline$\alpha 12_{1-5}$ & Dimer & $34 \pm 2.8$ \\
\hline$\beta 6^{*}{ }_{1-4}$ & Dimer & $16.3 \pm 2.1$ \\
\hline$\beta 8^{*}{ }_{1-4}$ & Dimer & $24.0 \pm 0.43$ \\
\hline$\gamma \mathrm{A} 1^{*}{ }_{1-4}$ & Dimer & $13.3 \pm 0.93$ \\
\hline$\gamma \mathrm{A} 4{ }^{*}{ }_{1-4}$ & Dimer & $45.3 \pm 1.52$ \\
\hline$\gamma \mathrm{A} 8^{*}{ }_{1-4}$ & Dimer & $30 \pm 1.5$ \\
\hline$\gamma A^{*}{ }_{1-5}$ & Dimer & $8.61 \pm 0.35$ \\
\hline$\gamma B 2^{*}{ }_{1-5}$ & Dimer & $21.8 \pm 0.21$ \\
\hline$\gamma \mathrm{B} 4_{1-5}$ & Dimer & $38 \pm 0.33$ \\
\hline$\gamma \mathrm{B}^{*}{ }_{1-4}$ & Dimer & $79.1 \pm 4.3$ \\
\hline$\gamma \mathrm{B} 5_{1-4}-\mathrm{AVI}$ & Dimer & $50 \pm 0.4$ \\
\hline$\alpha \mathrm{C} 2{ }^{*}{ }_{1-4}$ & Dimer & $20.6 \pm 1.19$ \\
\hline$\gamma C 3^{*}{ }_{1-4}$ & Dimer & $115 \pm 1.49\left(\mathrm{~K}_{\mathrm{i}} / \mathrm{K}_{\mathrm{D}}=1.57\right)$ \\
\hline$\gamma \mathrm{C} 4_{1-4}$ & Monomer / Very weak dimer & $>500^{\dagger}$ \\
\hline$\gamma C 5^{*}{ }_{1-5}$ & Dimer & $100 \pm 4.33$ \\
\hline \multicolumn{3}{|l|}{ Trans mutants } \\
\hline$\alpha 7_{1-5}$ L301R & Weakly dimeric & $490 \pm 57$ \\
\hline$\gamma \mathrm{A} 8_{1-4} \mathrm{I}^{116 \mathrm{R}^{*}}$ & Monomer & $N / A$ \\
\hline$\alpha C 2{ }_{1-3}$ & Dimer & $242 \pm 0.1 \quad\left(K_{i} / K_{D}=1.48\right)$ \\
\hline$\beta_{1-4} \mathrm{R} 41 \mathrm{~N}$ & Dimer & $160 \pm 0.38$ \\
\hline$\beta_{1-4} \mathrm{~S} 117 \mathrm{I}$ & Dimer & $72 \pm 34$ \\
\hline$\beta_{1-4} \mathrm{~L} 125 \mathrm{P}$ & Dimer & $150 \pm 20$ \\
\hline$\beta_{1-4}$ E369K & Dimer & $23 \pm 2.8$ \\
\hline$\beta_{1-4} \mathrm{Y} 371 \mathrm{~F}$ & Dimer & $39 \pm 5.6$ \\
\hline$\beta 1-4 \mathrm{R} 41 \mathrm{~N} / \mathrm{S} 117 \mathrm{I}$ & Precipitate & $\mathrm{N} / \mathrm{A}$ \\
\hline$\beta_{1-4} \mathrm{R} 41 \mathrm{~N} / \mathrm{E} 369 \mathrm{~K}$ & Dimer & $41 \pm 0.69$ \\
\hline$\beta_{1-4} \mathrm{~S} 117 \mathrm{I} / \mathrm{L} 125 \mathrm{P}$ & Dimer & $68 \pm 5.1$ \\
\hline$\beta_{1-4}$ R41N/S117I/L125P & Weak dimer & $350 \pm 11$ \\
\hline$\beta_{1-4}$ R41N/S117I/E369K & Dimer & $32 \pm 0.95$ \\
\hline$\beta_{1-4} \mathrm{R} 41 \mathrm{~N} / \mathrm{S} 117 \mathrm{I} / \mathrm{Y} 371 \mathrm{~F}$ & Dimer & $18 \pm 0.11$ \\
\hline$\beta_{1-4} \mathrm{R} 41 \mathrm{~N} / \mathrm{S} 117 \mathrm{I} / \mathrm{L} 125 \mathrm{P} / \mathrm{E} 369 \mathrm{~K} / \mathrm{Y} 371 \mathrm{~F}$ & Dimer & $63 \pm 11$ \\
\hline
\end{tabular}

Figure 2 - source data 1 . Sedimentation equilibrium analytical ultracentrifugation data for trans SPR reagents

* Previously published data (Rubinstein et al., 2015; Goodman et al., 2016a; Goodman et al., 2016b)

† Dissociation constants larger than $500 \mu \mathrm{M}$ cannot be accurately determined. 


\begin{tabular}{|c|c|c|c|}
\hline & \multicolumn{2}{|c|}{$\begin{array}{l}\text { CPcdh YC4 } \text { EC1-4 } \\
\text { crystal form } 1\end{array}$} & $\begin{array}{l}\text { cPcdh YC4 } \text { EC1-4 }_{\text {Fcr }} \\
\text { crystal form } 2\end{array}$ \\
\hline \multicolumn{4}{|l|}{ Data collection } \\
\hline Date & \multicolumn{2}{|c|}{ 02/07/2017 } & 03/04/2017 \\
\hline Beamline & \multicolumn{2}{|c|}{ APS 24-ID-E } & APS 24-ID-E \\
\hline Wavelength $(\AA)$ & \multicolumn{2}{|c|}{0.97918} & 0.97918 \\
\hline Space group & \multicolumn{2}{|c|}{$122_{1} 2_{1}$} & $P 2{ }_{1}{ }_{1} 2_{1}$ \\
\hline \multicolumn{4}{|l|}{ Cell dimensions } \\
\hline$a, b, c(\AA)$ & \multicolumn{2}{|c|}{$75.220,115.680,194.480$} & $51.944,103.948,200.592$ \\
\hline \multirow[t]{2}{*}{$\alpha, \beta, \gamma\left({ }^{\circ}\right)$} & \multicolumn{2}{|c|}{$90,90,90$} & $90,90,90$ \\
\hline & Spherical resolution limits & Ellipsoidal resolution limits & \\
\hline Resolution $(\AA)$ & $40.00-3.50(3.83-3.50)$ & $40-4.6 / 3.9 / 3.5(3.84-3.51)$ & $40.00-2.40(2.49-2.40)$ \\
\hline No. of reflections & 79877 (19206) & $53652(2402)$ & $240907(16849)$ \\
\hline Unique reflections & $11071(2608)$ & $7545(340)$ & $42979(4175)$ \\
\hline$R_{\text {merge }}$ & $0.381(3.459)$ & $0.215(0.830)$ & $0.060(0.438)$ \\
\hline$R_{\text {meas }}$ & $0.411(3.722)$ & $0.232(0.896)$ & $0.073(0.560)$ \\
\hline $\mathrm{R}_{\text {pim }}$ & $0.152(1.366)$ & $0.086(0.335)$ & $0.040(0.342)$ \\
\hline $\operatorname{CC}(1 / 2)$ & $0.995(0.533)$ & $0.997(0.829)$ & $0.999(0.924)$ \\
\hline$|/ \sigma|$ & $5.7(0.8)$ & $8.3(2.9)$ & $13.3(2.5)$ \\
\hline $\begin{array}{l}\text { Spherical } \\
\text { completeness (\%) }\end{array}$ & $99.9(100.0)$ & $67.6(12.5)$ & $98.9(92.6)$ \\
\hline $\begin{array}{l}\text { Ellipsoidal } \\
\text { completeness (\%) }\end{array}$ & N/A & 96.8 & N/A \\
\hline Redundancy & $7.2(7.4)$ & $7.1(7.1)$ & $5.6(4.0)$ \\
\hline \multicolumn{4}{|l|}{ Refinement } \\
\hline$\overline{\text { Resolution }(\AA)}$ & \multicolumn{2}{|c|}{$40-4.6 / 3.9 / 3.5$} & $40.00-2.40$ \\
\hline Unique reflections & \multicolumn{2}{|c|}{7545} & 42815 \\
\hline $\begin{array}{l}\text { Completeness in } \\
\text { diffracting sphere or } \\
\text { ellipsoid* }(\%)\end{array}$ & \multicolumn{2}{|c|}{$96.8^{*}$} & 98.5 \\
\hline$R_{\text {work }} / R_{\text {free }}(\%)$ & \multicolumn{2}{|c|}{$22.3 / 27.1$} & $19.6 / 24.1$ \\
\hline Molecules in A.S.U. & \multicolumn{2}{|c|}{1} & 2 \\
\hline Number of atoms & \multicolumn{2}{|c|}{3242} & 6736 \\
\hline Protein & \multicolumn{2}{|c|}{3120} & 6327 \\
\hline Ligand/Ion & \multicolumn{2}{|c|}{122} & 284 \\
\hline Water & \multicolumn{2}{|c|}{0} & 125 \\
\hline B-factors & \multicolumn{2}{|c|}{101.24} & 73.39 \\
\hline Protein & \multicolumn{2}{|c|}{101.03} & 72.99 \\
\hline Ligand/lon & \multicolumn{2}{|c|}{106.73} & 88.54 \\
\hline Water & \multicolumn{2}{|c|}{$\mathrm{N} / \mathrm{A}$} & 59.26 \\
\hline \multicolumn{4}{|l|}{ R.m.s. deviations } \\
\hline Bond lengths $(\AA)$ & & & 0.003 \\
\hline Bond angles $\left({ }^{\circ}\right)$ & & & 0.653 \\
\hline Ramachandran & & & \\
\hline Favored (\%) & & & 98.92 \\
\hline Allowed $(\%)$ & & & 1.08 \\
\hline Outliers (\%) & & & 0.00 \\
\hline Rotamer outliers (\%) & & & 0.57 \\
\hline Wilson B & & & 51.79 \\
\hline PDB ID & & & 7RGF \\
\hline
\end{tabular}

Figure 3 - source data 1. X-ray crystallography data collection and refinement statistics

Values in parentheses are for the outer shell. APS, Advanced Photon Source, Argonne National Lab; A.S.U., asymmetric unit; R.m.s., Root mean square. See Figure 4-figure supplement 1 and Methods for further details on the ellipsoidal resolution limits. 


\begin{tabular}{|c|c|c|c|}
\hline \multirow{2}{*}{$\begin{array}{l}\text { RMSDs over trans dimers and individual } \\
\text { interacting domains }\end{array}$} & \multicolumn{3}{|c|}{$\gamma \mathrm{C} 4$ crystal form 1 (intact interface) } \\
\hline & $\begin{array}{l}\text { EC1-4:EC1-4 (834 } \\
\text { atoms) }\end{array}$ & $\begin{array}{l}\text { EC1:EC4 (196 } \\
\text { atoms) }\end{array}$ & $\begin{array}{l}\text { EC2:EC3 (209 } \\
\quad \text { atoms) }\end{array}$ \\
\hline$\gamma \mathrm{C} 4$ crystal form 2 chain $\mathrm{A}$ :chain $\mathrm{B}$ & $2.9 \AA$ (734 atoms) & $1.3 \AA$ (196 atoms) & $0.9 \AA$ (198 atoms) \\
\hline$\gamma \mathrm{C} 4$ crystal form 2 chain B:chain A & $2.9 \AA$ (734 atoms) & $1.9 \AA$ (194 atoms) & $1.3 \AA$ (207 atoms) \\
\hline \multicolumn{4}{|l|}{ Alternate clustered Pcdhs: } \\
\hline$\alpha 7$ dimer & $3.3 \AA$ (801 atoms) & $2.0 \AA$ (189 atoms) & $1.7 \AA$ (196 atoms) \\
\hline$\beta 6$ dimer & $4.5 \AA$ (769 atoms) & $2.5 \AA$ (180 atoms) & $3.8 \AA$ (200 atoms) \\
\hline$\gamma \mathrm{A} 1$ dimer & $3.5 \AA$ (794 atoms) & $3.2 \AA$ (191 atoms) & $2.2 \AA$ (182 atoms) \\
\hline$\gamma \mathrm{B} 2$ dimer & $4.3 \AA$ (802 atoms) & $1.5 \AA$ (187 atoms) & $1.8 \AA$ (196 atoms) \\
\hline \multicolumn{4}{|l|}{ Non-clustered $\delta 1$ Pcdhs: } \\
\hline Human ncPcdh1 dimer & $7.0 \AA$ (784 atoms) & $1.5 \AA$ (139 atoms) & $2.9 \AA$ (185 atoms) \\
\hline \multicolumn{4}{|l|}{ Non-clustered $\delta 2$ Pcdhs: } \\
\hline Xenopus ncPcdh8.1 dimer & $3.4 \AA$ (756 atoms) & $3.0 \AA$ (174 atoms) & $2.4 \AA$ (206 atoms) \\
\hline Human ncPcdh10 dimer & $2.4 \AA$ (785 atoms) & $2.1 \AA$ (189 atoms) & $1.3 \AA$ (195 atoms) \\
\hline Human ncPcdh18 dimer & $3.1 \AA$ (786 atoms) & $1.7 \AA$ (157 atoms) & $2.4 \AA$ (207 atoms) \\
\hline Human ncPcdh19 dimer & $3.3 \AA$ (780 atoms) & $2.0 \AA$ (155 atoms) & $1.2 \AA$ (187 atoms) \\
\hline Zebrafish ncPcdh19 dimer & $2.4 \AA$ (778 atoms) & $1.9 \AA$ (165 atoms) & $1.2 \AA$ (198 atoms) \\
\hline
\end{tabular}

Figure 3 - source data 2 . Overall structural similarity between $\gamma \mathrm{C} 4$, alternate cPcdhs, and nonclustered Pcdhs trans dimer structures

Table lists the pairwise root mean square deviations over aligned C $\alpha$ 's (RMSDs) between the intact $\gamma \mathrm{C}_{\mathrm{ECl}-4}$ trans dimer (crystal form 1) and a representative selection of available cPcdh and ncPcdh trans dimer structures. RMSDs between the complete EC1-4:EC1-4 trans dimers are given in column 2. RMSDs between individual interacting EC1:EC4 and EC2:EC3 regions of Pcdh trans-dimer structures are given in columns 3 and 4. The number of aligned $C \alpha$ 's for each pairwise alignment is given in parentheses. The PDB codes for the aligned crystal structures are as follows: $\alpha 7_{\mathrm{EC} 1-5}, 5 \mathrm{DZV} ; \beta 6_{\mathrm{EC} 1-4}$, 5DZX; $\beta 8_{\mathrm{EC} 1-4}, 5 \mathrm{SZL} ; \gamma \mathrm{B} 2_{\mathrm{EC} 1-5}, 5 \mathrm{~T} 9 \mathrm{~T} ; \alpha \mathrm{C} 2_{\mathrm{EC} 1-3}, 4 \mathrm{ZPM} ; \gamma \mathrm{C} 3_{\mathrm{EC} 1-3}, 4 Z \mathrm{ZI} ; \gamma \mathrm{C} 5_{\mathrm{EC} 1-3}, 4 Z \mathrm{ZO} ;$ ncPcdh $1_{\mathrm{EC} 1-4}$, 6MGA; ncPcdh8.1 $1_{\mathrm{EC} 1-6}, 6 \mathrm{VG1}$; ncPcdh10 ${ }_{\mathrm{EC} 1-4}, 6 \mathrm{VFW}$; ncPcdh18 $8_{\mathrm{EC} 1-4}, 6 \mathrm{VFR}$; ncPcdh19 $9_{\mathrm{EC} 1-4}, 6 \mathrm{VFU}$; and zebrafish ncPcdh199 $9_{\mathrm{EC} 1-4}$, $5 \mathrm{IU} 9$ (Goodman et al., 2016a; Goodman et al., 2016b; Rubinstein et al., 2015; Nicoludis et al., 2015; Modak and Sotomayor, 2019; Harrison et al., 2020; Cooper et al., 2016) 


\begin{tabular}{|c|c|c|}
\hline Protein & Oligomeric State & Dissociation Constant, $\mathrm{K}_{\mathrm{D}}(\mu \mathrm{M})$ \\
\hline \multicolumn{3}{|c|}{ Cis-interacting fragments } \\
\hline $\begin{array}{l}\beta 1_{3-6} \\
\beta 6_{1-6} \\
\beta 9_{3-6} \\
\gamma \mathrm{A} 3_{3-6} \\
\gamma \mathrm{A} 4^{*}{ }_{3-6} \\
\gamma \mathrm{A} 9_{3-6} \\
\gamma \mathrm{B} 2^{*}{ }_{3-6} \\
\gamma \mathrm{B} 5^{\star}{ }_{3-6} \\
\gamma \mathrm{B} 7^{*}{ }^{*-6} \\
\alpha \mathrm{C} 2_{3-6}-\mathrm{AVI} \\
\alpha C 2^{*}{ }_{2-6} \\
\alpha 7_{1-5} / \gamma \mathrm{C} 3_{6} \text { chimera }^{*} \\
\gamma \mathrm{C} 3^{*}{ }_{3-6} \\
\gamma \mathrm{C} 5_{2-6}\end{array}$ & $\begin{array}{l}\text { Monomer } \\
\text { Tetramer } \\
\text { Dimer } \\
\text { Dimer } \\
\text { Monomer } \\
\text { Monomer } \\
\text { Dimer } \\
\text { Dimer } \\
\text { Dimer } \\
\text { Dimer } \\
\text { Dimer } \\
\text { Tetramer } \\
\text { Monomer } \\
\text { Dimer }\end{array}$ & $\begin{array}{c}\mathrm{N} / \mathrm{A} \\
1.7 / 12.1^{\dagger} \\
35 \pm 3.1 \\
110 \pm 7.3 \\
\mathrm{~N} / \mathrm{A} \\
\mathrm{N} / \mathrm{A} \\
80.1 \pm 12.8 \\
32.6 \pm 4.6 \\
59.0 \pm 3.4 \\
7.2 \pm 1.2 \\
8.92 \pm 0.28 \\
3.0 / 3.9^{\dagger} \\
\mathrm{N} / \mathrm{A} \\
18.4 \pm 0.24\end{array}$ \\
\hline $\begin{array}{l}\text { Cis mutants } \\
\gamma B 7_{3-6} Y 532 G^{*} \\
\gamma B 7_{3-6} A 570 R\end{array}$ & $\begin{array}{l}\text { Monomer } \\
\text { Monomer }\end{array}$ & $\begin{array}{l}N / A \\
N / A\end{array}$ \\
\hline
\end{tabular}

Figure 4-source data 1. Sedimentation equilibrium analytical ultracentrifugation data for cis SPR reagents

* Previously published data (Rubinstein et al., 2015; Goodman et al., 2016b; Goodman et al., 2017)

$+\mathrm{K}_{\mathrm{D}} \mathrm{S}$ of monomer-to-dimer / dimer-to-tetramer transitions from fitting the data to a tetramer model. 

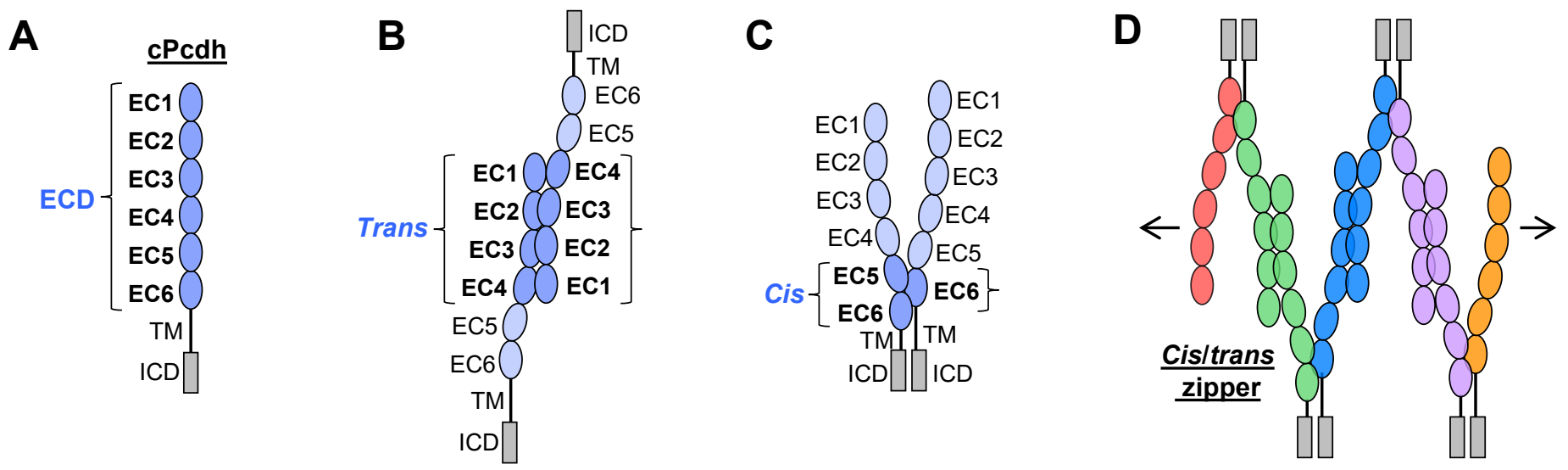

Figure 1: cPedh domain organization and extracellular interactions

(A) Schematic depicting the domain organization of cPcdhs. EC, extracellular cadherin domain; TM, transmembrane domain; ECD, ectodomain; ICD, intracellular domain.

(B) Schematic of two cPcdhs interacting via the EC1-4 trans interface.

(C) Schematic of two cPcdhs interacting via the EC5-6/EC6 cis interface.

(D) Schematic depiction of the cis/trans cPcdh zipper comprising multiple cPcdh isoforms (various colors) engaged in homophilic trans interactions and promiscuous cis interactions as required for the proposed "isoform-mismatch chaintermination model" of cPcdh-mediated neuronal self-recognition and self-avoidance. 


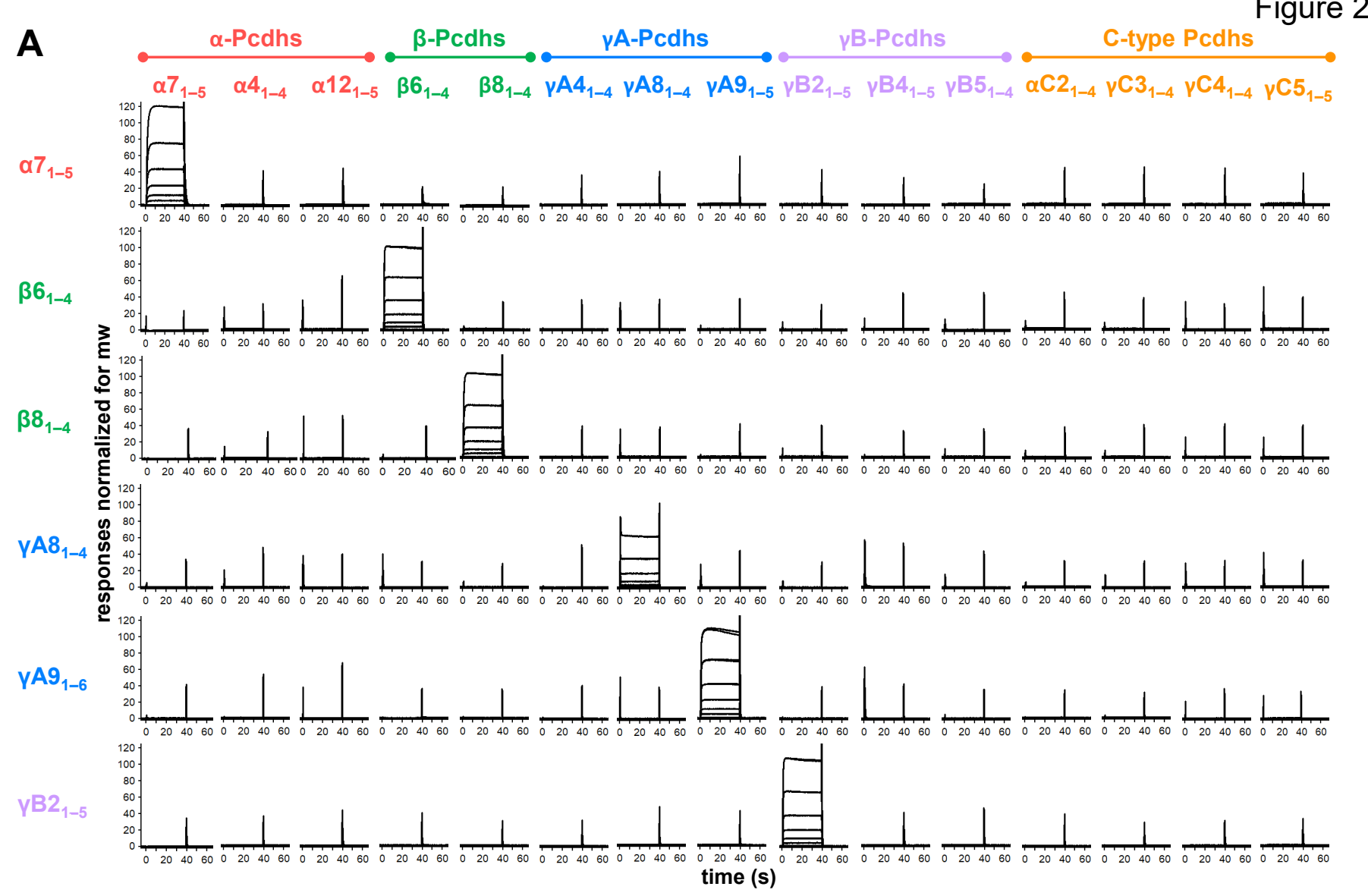

B

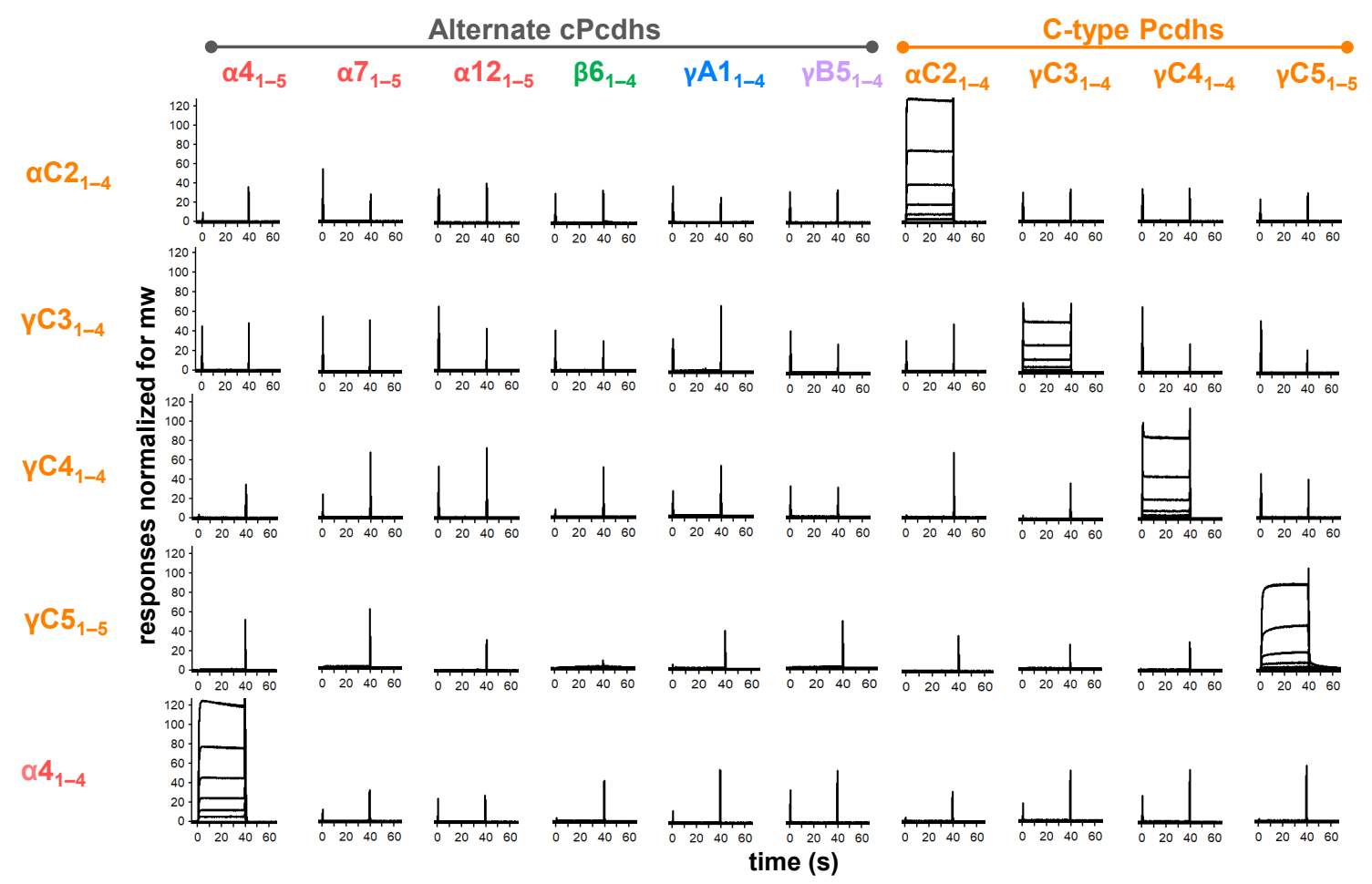

Figure 2: cPcdhs show strict homophilic specificity in their trans interactions

(A) SPR binding profiles of cPcdh trans fragment analytes from all cPcdh subfamilies (denoted in the top row) flowed over six surfaces coated with alternate cPcdh trans fragments (rows). Responses over all surfaces are drawn on the same scale and normalized for molecular weight.

(B) SPR binding profiles of cPcdh trans fragment analytes from all cPcdh subfamilies (shown in columns) flowed over individual surfaces coated with C-type and $\alpha 4 \mathrm{cPcdh}$ trans fragments (rows). Responses over all surfaces are drawn on the same scale and normalized for molecular weight. 
A

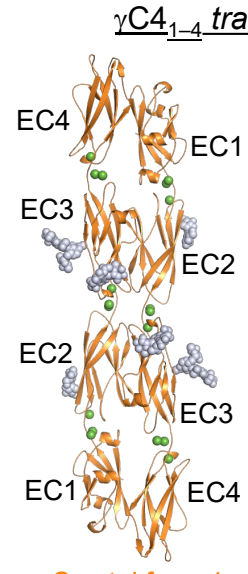

Crystal form 1

PDB 7JGZ

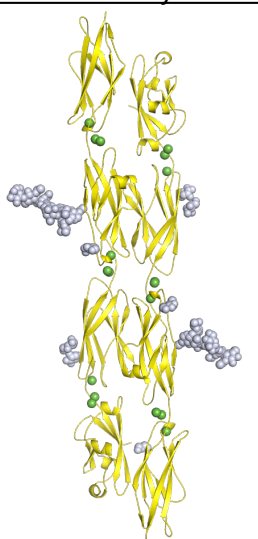

Crystal form 2 PDB 7RGF

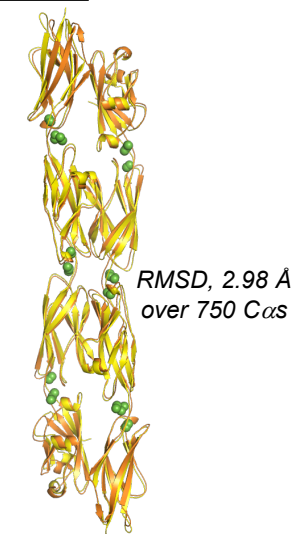

Superposition
B Interfacial differences between the $\mathrm{CC}_{\underline{1-4}}$ structures

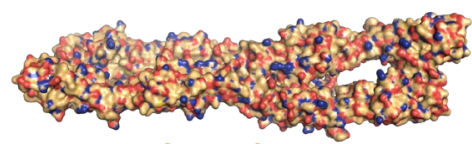

Crystal form 1

$\mathrm{BSA}=3800 \AA^{2}$

(intact interface)

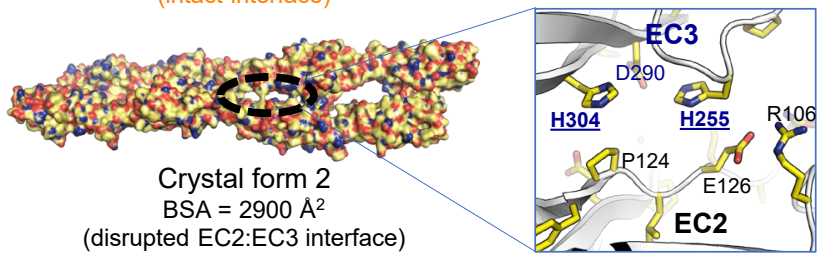

C

Comparison between specific regions of the $\gamma \mathrm{C} 4$ and $\gamma \mathrm{B} 2$ trans dimer interfaces
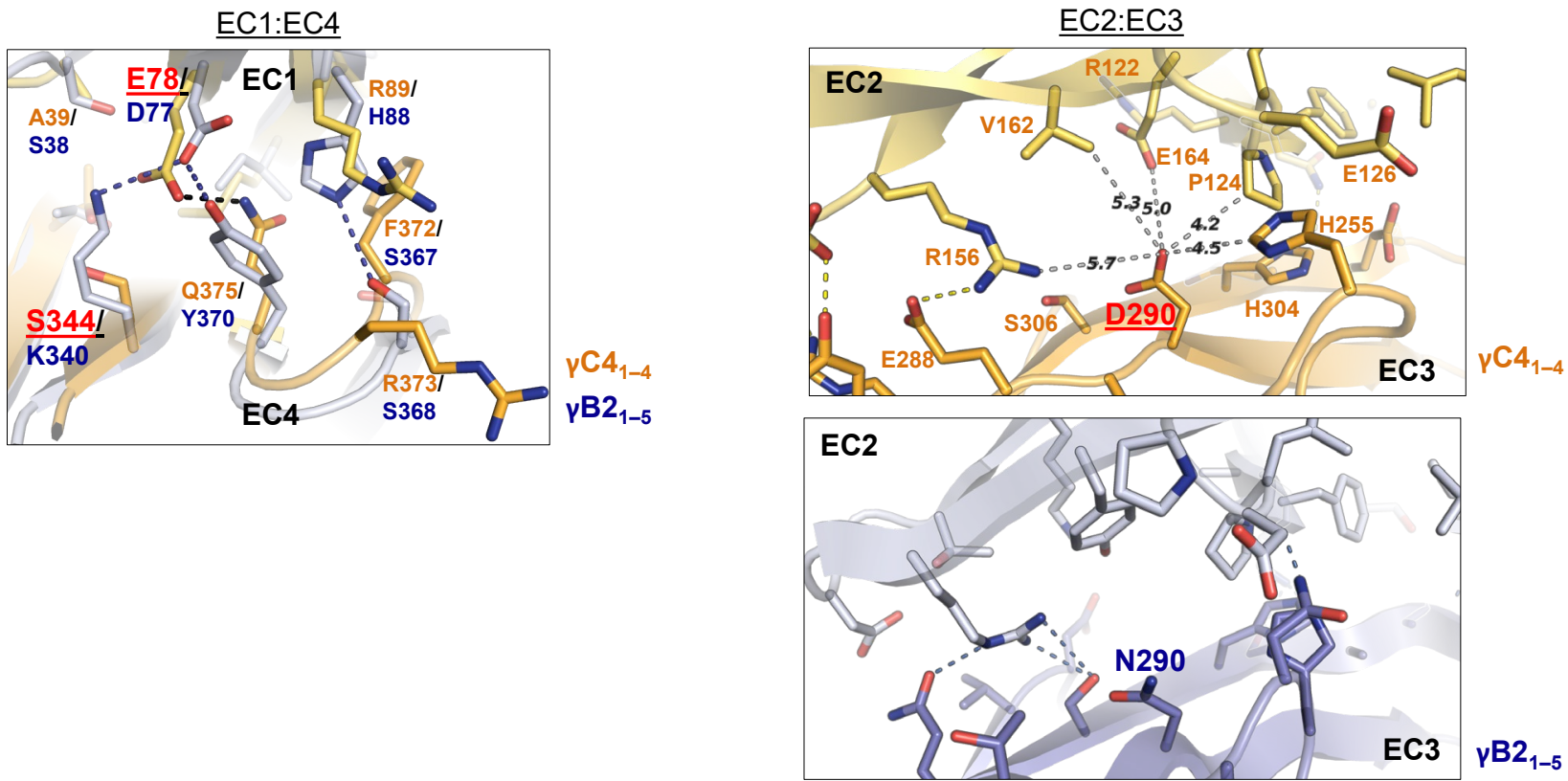

$\gamma B 2_{1-5}$

D

\begin{tabular}{|l|c|c|}
\hline Protein & Oligomeric State & $\begin{array}{c}\text { Dissociation } \\
\text { Constant, } \mathbf{K}_{\mathrm{D}}(\boldsymbol{\mu} \mathrm{M})\end{array}$ \\
\hline$\gamma \mathrm{C}_{1-4} \mathrm{wt}$ & $\begin{array}{c}\text { Monomer / Very } \\
\text { weak dimer }\end{array}$ & $>500$ \\
\hline$\gamma \mathrm{C}_{1-4} \mathrm{E} 78 \mathrm{~A}$ & Dimer & $58 \pm 0.60$ \\
\hline$\gamma \mathrm{C} 4_{1-4} \mathrm{E} 78 \mathrm{Q}$ & Dimer & $83 \pm 2.6$ \\
\hline$\gamma \mathrm{C}_{1-4} \mathrm{~S} 344 \mathrm{R}$ & Dimer & $112 \pm 14$ \\
\hline$\gamma \mathrm{C} 4_{1-4} \mathrm{D} 290 \mathrm{~A}$ & Monomer & $\mathrm{N} / \mathrm{A}$ \\
\hline$\gamma \mathrm{C} 4_{1-4} \mathrm{D} 290 \mathrm{~N}$ & Monomer & $\mathrm{N} / \mathrm{A}$ \\
\hline
\end{tabular}


Figure 3: C-type cPcdh $\gamma \mathrm{C} 4$ adopts an EC1-4-mediated head-to-tail trans dimer like alternate cPcdhs with a comparatively weak dimer affinity

(A) Ribbon diagrams of the $\gamma \mathrm{C}_{\mathrm{ECl}-4}$ trans dimer crystal structures obtained from two different crystal forms. Bound calcium ions are shown as green spheres and glycans are shown in pale blue spheres.

(B) The two crystal structures have a markedly different trans interface buried surface area (BSA). Left, Surface views of the two trans dimer crystal structures highlight the difference, with a gap apparent in the EC2:EC3 region of the interface in crystal form 2 that is absent from crystal form 1 . Surfaces are colored by atom type with the carbons colored orange for crystal form 1 and yellow for crystal form 2. Right, Close up view of the gap region in the crystal form 2 dimer with the side chains depicted as sticks. The intact crystal form $1 \gamma \mathrm{C} 4$ dimer is similar overall to those of the published intact alternate $\alpha, \beta, \gamma \mathrm{A}$, and $\gamma \mathrm{B}$ cPcdhs and the published $\delta 2$ non-clustered (nc) Pcdh trans dimers (root mean square deviation over aligned Cas (RMSD) 2.4 $4.5 \AA$; Figure 3-source data 2). The published crystal structures of $\gamma \mathrm{A} 8, \gamma \mathrm{A} 1$, and $\gamma \mathrm{B} 3$ also show partially disrupted trans interfaces though in differing regions of the interface (Goodman et al., 2016b, Nicoludis et al., 2016).

(C) Comparison between the (i) EC1:EC4 and (ii) EC2:EC3 regions of the $\gamma \mathrm{C} 4$ (orange) and $\gamma \mathrm{B} 2$ (blue, PDB 5T9T) trans dimer interfaces. (i) Structural alignment of the EC1:EC4 portion of the $\gamma \mathrm{C} 4$ and $\gamma \mathrm{B} 2$ trans dimers highlights a possible destabilizing role for $\gamma \mathrm{C} 4$ residue E78 since unlike its counterpart in $\gamma \mathrm{B} 2$ (D77) it is not juxtaposed with a basic residue. (ii) Similarly, an additional negatively charged residue (D290) which occupies a central position in the $\gamma \mathrm{C} 4 \mathrm{EC} 2$ :EC3 interface may also contribute to $\gamma \mathrm{C} 4$ 's comparatively weak trans dimer interaction. Distances between the D290 side chain and its nearest contacts are shown as dashed grey lines with distances given in Angstroms.

(D) Sedimentation equilibrium AUC experiments were conducted on $\gamma \mathrm{C} 4 \mathrm{EC} 1-4$ wild type (wt) and interface mutants to assess whether E78 and D290 negatively impact trans dimerization. Table details the oligomeric state and dissociation constants for each protein tested. 


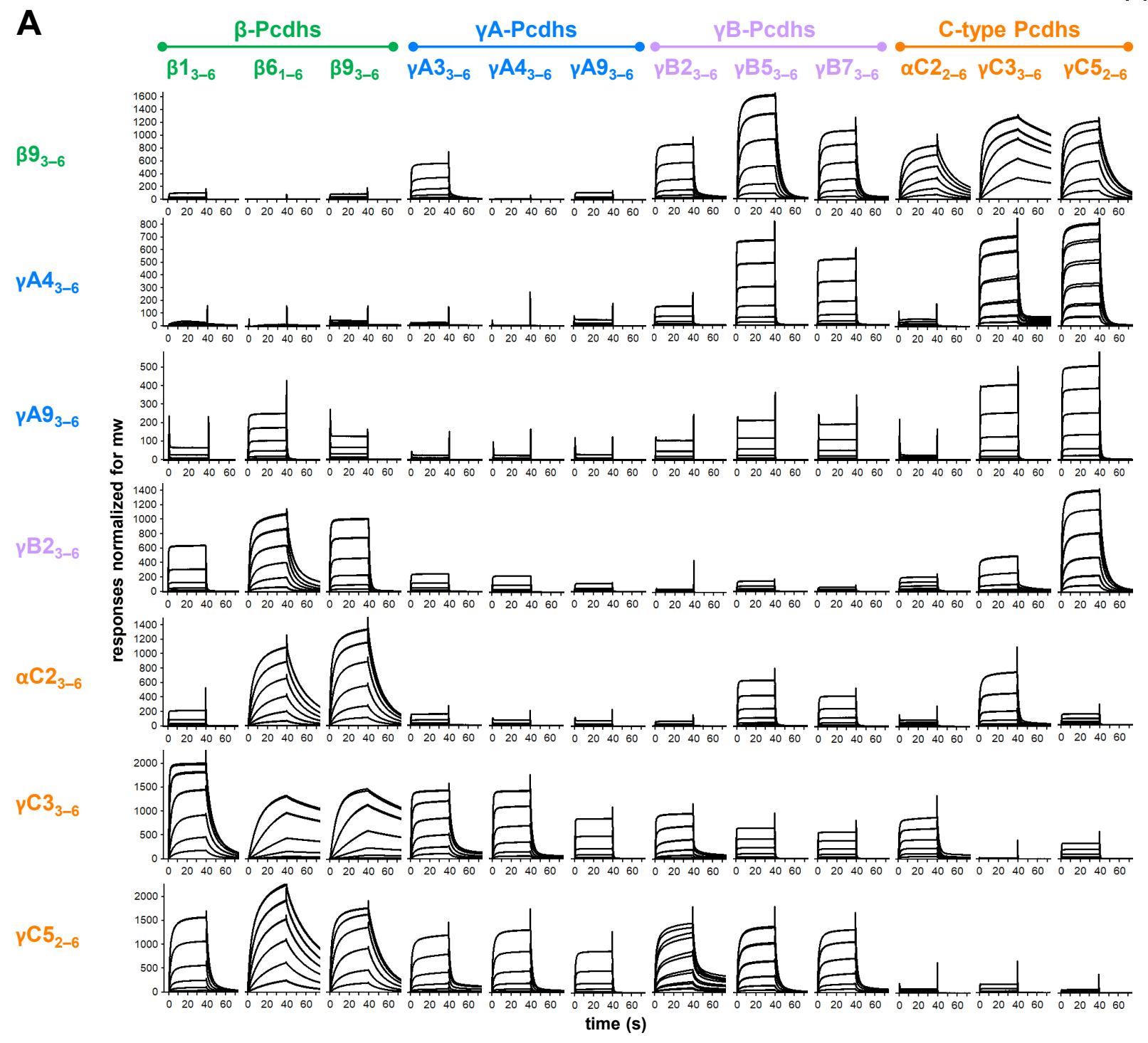

B

\begin{tabular}{|c|c|c|c|c|}
\hline & \multicolumn{4}{|c|}{$\mathrm{K}_{\mathrm{D}}$ for heterophilic cis interactions $(\mu \mathrm{M})$} \\
\hline Cis dimer & $\beta 1_{3-6}$ & $\gamma \mathrm{A}_{3-6}$ & $\gamma \mathrm{A} 9_{3-6}$ & $\gamma \mathrm{C}_{3-6}$ \\
\hline$\beta 9_{3-6}$ & $\mathrm{NB}$ & $\mathrm{NB}$ & $\mathrm{NB}$ & $0.2201(2)$ \\
\hline$\gamma \mathrm{A4} 4_{3-6}$ & $>50$ & & $>50$ & $2.73(1)$ \\
\hline$\gamma \mathrm{A} 9_{3-6}$ & $>50$ & $>50$ & & $9.60(3)$ \\
\hline$\gamma \mathrm{B}_{3-6}$ & $25.69(8)$ & $44.0(6)$ & $>50$ & $20.00(7)$ \\
\hline$\alpha \mathrm{C}_{3-6}$ & $>50$ & $>50$ & $>50$ & $11.51(1)$ \\
\hline$\gamma \mathrm{C}_{3-6}$ & $1.116(4)$ & $3.47(2)$ & $14.5(2)$ & \\
\hline$\gamma \mathrm{C}_{2-6}$ & $6.91(3)$ & $7.74(4)$ & $18.2(2)$ & $>50$ \\
\hline
\end{tabular}

Figure 4: cPcdh cis interactions are promiscuous with a preference for interfamily heterodimers

(A) SPR binding profiles of cPcdh cis fragment analytes from all cPcdh subfamilies except alphas (shown in columns) flowed over individual surfaces coated with cPcdh cis fragments. Binding profiles for each surface are individually scaled and responses are normalized for molecular weight.

(B) Table of dissociation constants calculated from the SPR data for the four monomeric analytes. The number in brackets represents the error of the fit based on analysis of duplicate responses. Binding signals were not detected for interactions labeled $\mathrm{NB}$, while $>50$, represents interactions with $\mathrm{K}_{\mathrm{D}} \mathrm{s}>50 \mu \mathrm{M}$, where an accurate $\mathrm{K}_{\mathrm{D}}$ cannot be determined. 
A

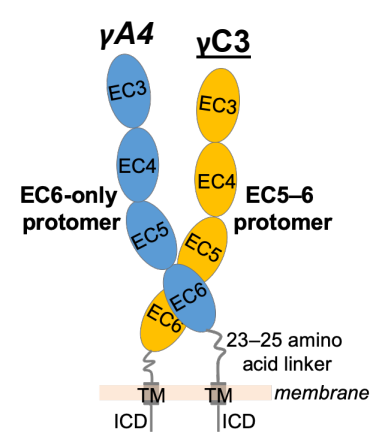

B
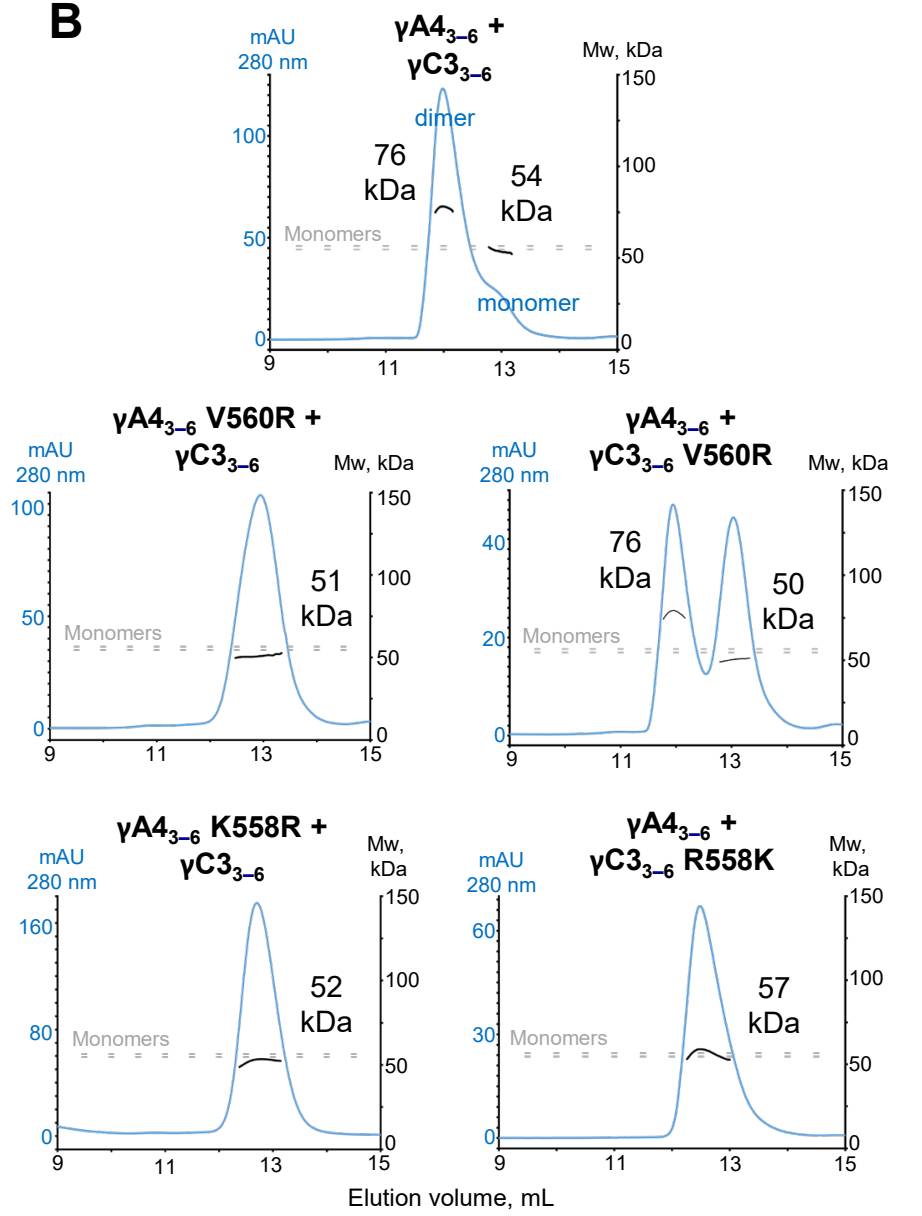

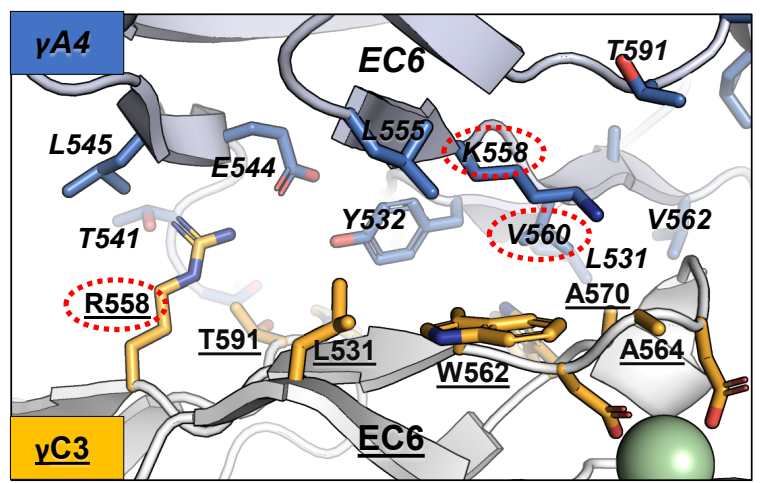

C

Figure 5

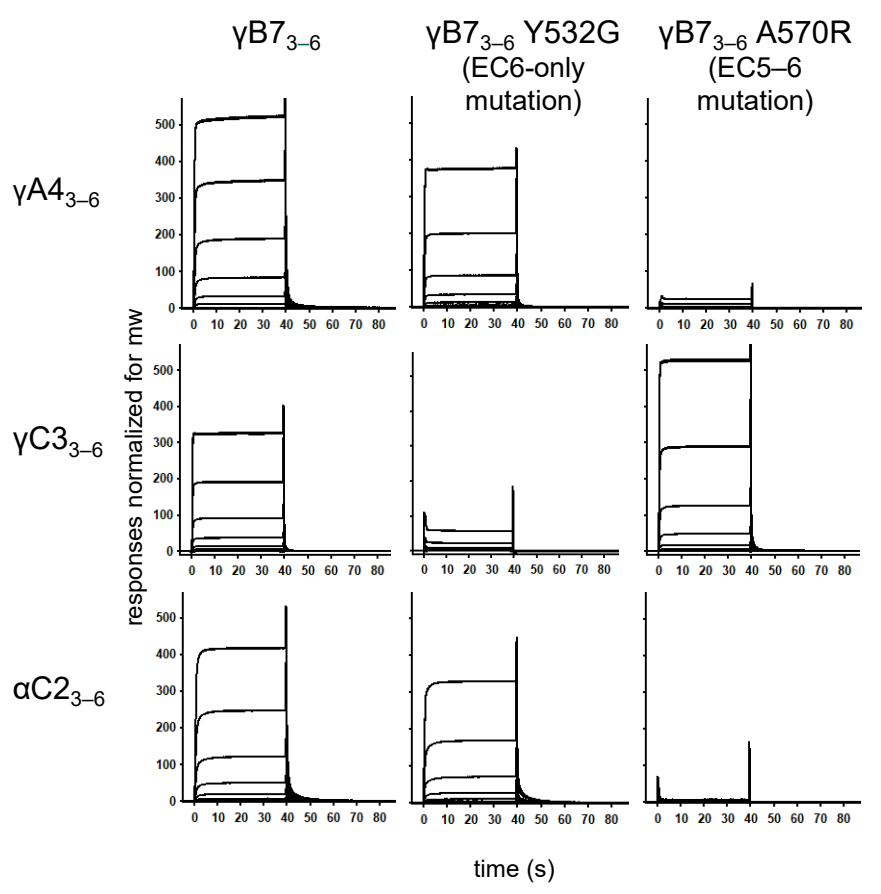

Figure 5: $\gamma \mathrm{A} 4$ preferentially forms the EC6-only side and $\gamma \mathrm{C3}$ the EC5-6 side in cis dimers

(A) Structural model of $\gamma \mathrm{A} 4 / \gamma \mathrm{C} 3$ cis dimer based on $\gamma \mathrm{B} 7_{\mathrm{EC} 3-6}$ cis dimer and $\gamma \mathrm{A}_{\mathrm{EC} 3-6}$ crystal structures (PDBs: 5V5X and 5SZQ). $\gamma \mathrm{A} 4$ is shown adopting the EC6-only side (blue protomer) and $\gamma \mathrm{C} 3$ is shown adopting the EC5-6 side (yellow protomer). Left, schematic of the $\gamma \mathrm{A} 4 / \gamma \mathrm{C} 3 \mathrm{EC} 3-6$ cis dimer. Right, close-up view of the EC6:EC6 interface from the modeled cis dimer showing interfacial residue side chains. Bound calcium ions are shown as green spheres. Residues which were mutated in the panel $\mathrm{B}$ are circled in red. $\gamma \mathrm{B} 7$ crystal structure numbering is used for both $\gamma \mathrm{A} 4$ and $\gamma \mathrm{C} 3$ residues. See methods for $\gamma \mathrm{A} 4$ and $\gamma \mathrm{C} 3$ alignment. Please note the model shown here is solely for hypothesis generation, since it is unlikely to be completely accurate. See methods for further details of structural modeling. (B) Top, SEC-MALS data for an equimolar mixture of wild-type $\gamma \mathrm{A}_{\mathrm{EC} 3-6}$ and $\gamma \mathrm{C} 3_{\mathrm{EC} 3-6}$ showing dimer formation. Plot shows size exclusion absorbance at $280 \mathrm{~nm}$ trace (left axis), molecular weight of the eluant peaks (right axis), and the monomer molecular weights of $\gamma \mathrm{A}_{\mathrm{EC}^{-6}-6}$ and $\gamma \mathrm{C} 3_{\mathrm{EC} 3-6}$ measured by mass spectrometry $-54.5 \mathrm{kDa}$ and $56.5 \mathrm{kDa}$ respectively - as dashed grey lines. Average molecular weight of the molecules in the dimer and monomer eluant peaks are labeled. Middle, SEC-MALS data for V560R mutants, which target the EC6-only side of the interface. Bottom, SEC-MALS data for residue 558 mutants. The $\gamma$ C3-like K558R mutation in $\gamma$ A4 inhibits heterodimer formation with wild-type $\gamma \mathrm{C} 3$. Similarly, the $\gamma \mathrm{A} 4$-like R558K in $\gamma \mathrm{C} 3$ inhibits dimerization with wild-type $\gamma \mathrm{A} 4$.

(C) SPR binding profiles for $\gamma \mathrm{B} 7_{\mathrm{EC} 3-6}$ wild type and cis interface mutants flowed over three individual wild-type cis fragment surfaces. The two mutations specifically target one side of the cis interface. 
Figure 2-figure supplement 1
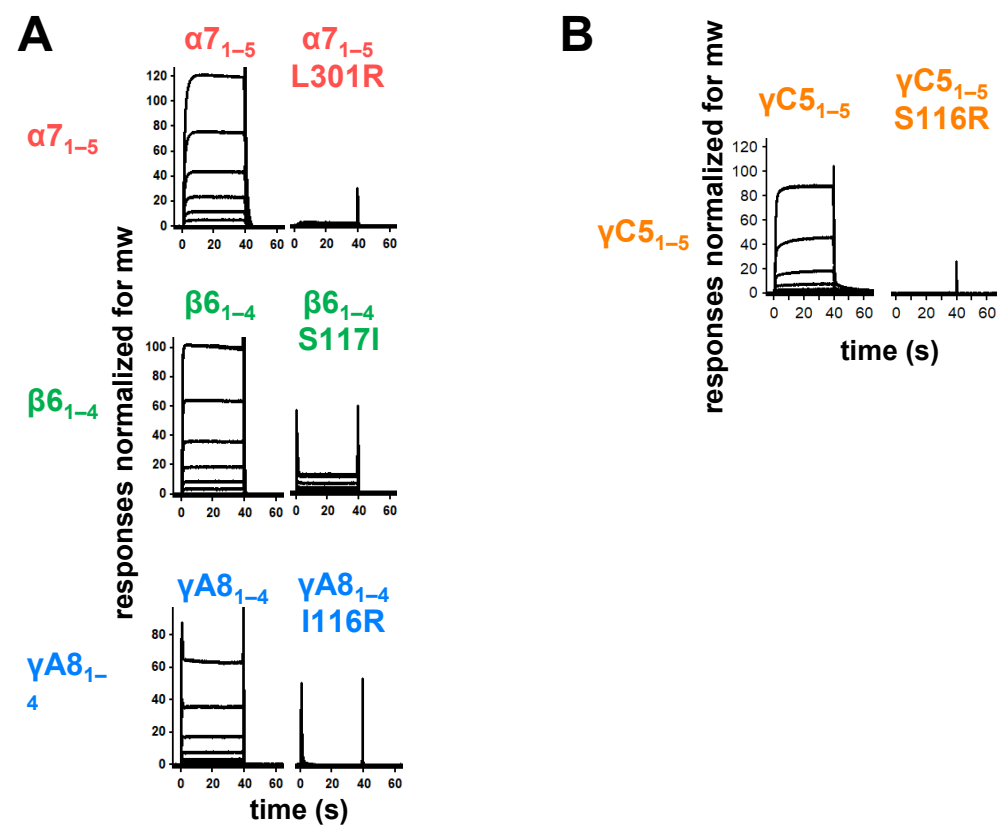

Figure 2-figure supplement 1: Trans interface mutants demonstrate homophilic interactions observed in SPR are mediated by the trans dimer interface

(A) SPR binding curves for wild-type and trans mutant alternate cPcdhs flowed over their respective immobilized wild-type molecule.

(B) SPR binding curves for wild-type and trans mutant C-type cPcdh $\gamma \mathrm{C} 5$ flowed over immobilized wild-type $\gamma \mathrm{C} 5$. 
A
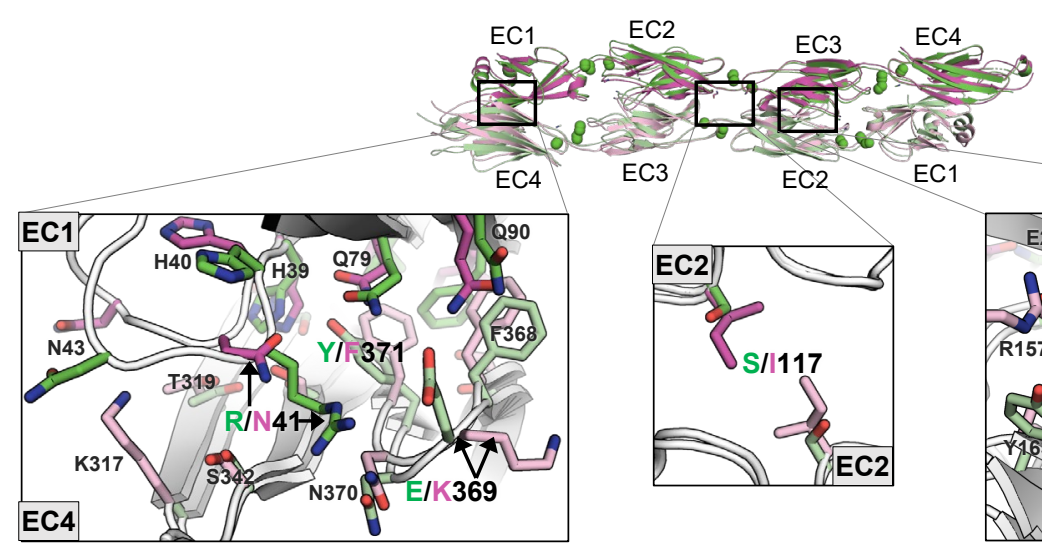

Figure 2-figure supplement 2

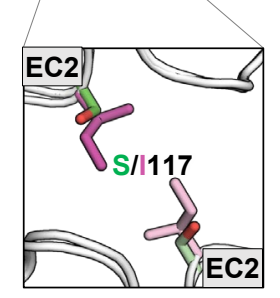

$\beta 6_{1-4}$

$\beta 8_{1-4}$

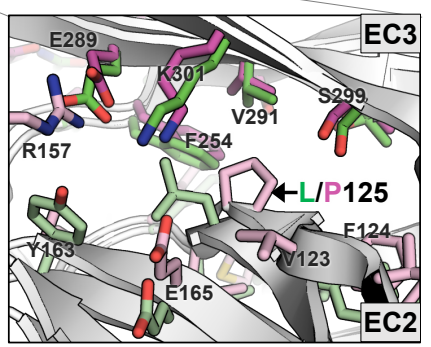

\section{B}

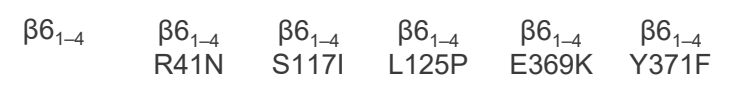

$\begin{array}{cc}\beta 6_{1-4} & \beta 6_{1-4} \\ \text { R41N/ } & \text { R41N/ } \\ \text { E369K } & \text { S117I }\end{array}$

$\beta 6_{1-4}$

S117I/

$\beta 6_{1-4}$
$\mathrm{R} 41 \mathrm{~N} /$

$\beta 6_{1-4}$

$\beta 6_{1-4}$
$\mathrm{R} 41 \mathrm{~N} /$

L125P E369K/

L125P E369K

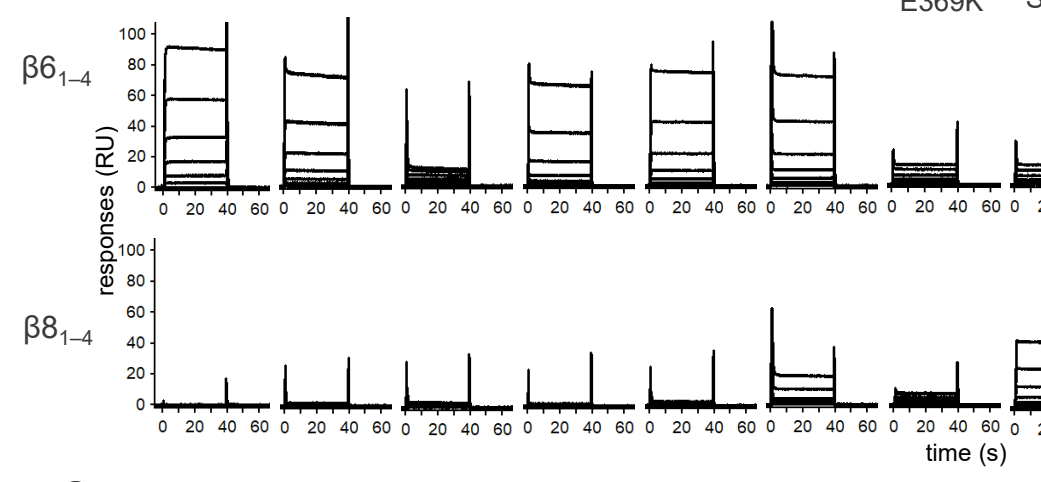

\section{C}

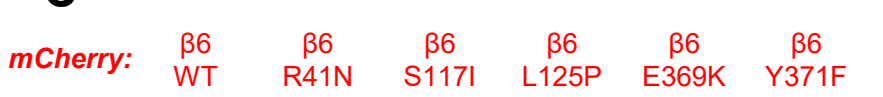

mVenus: $\beta 6$ WT
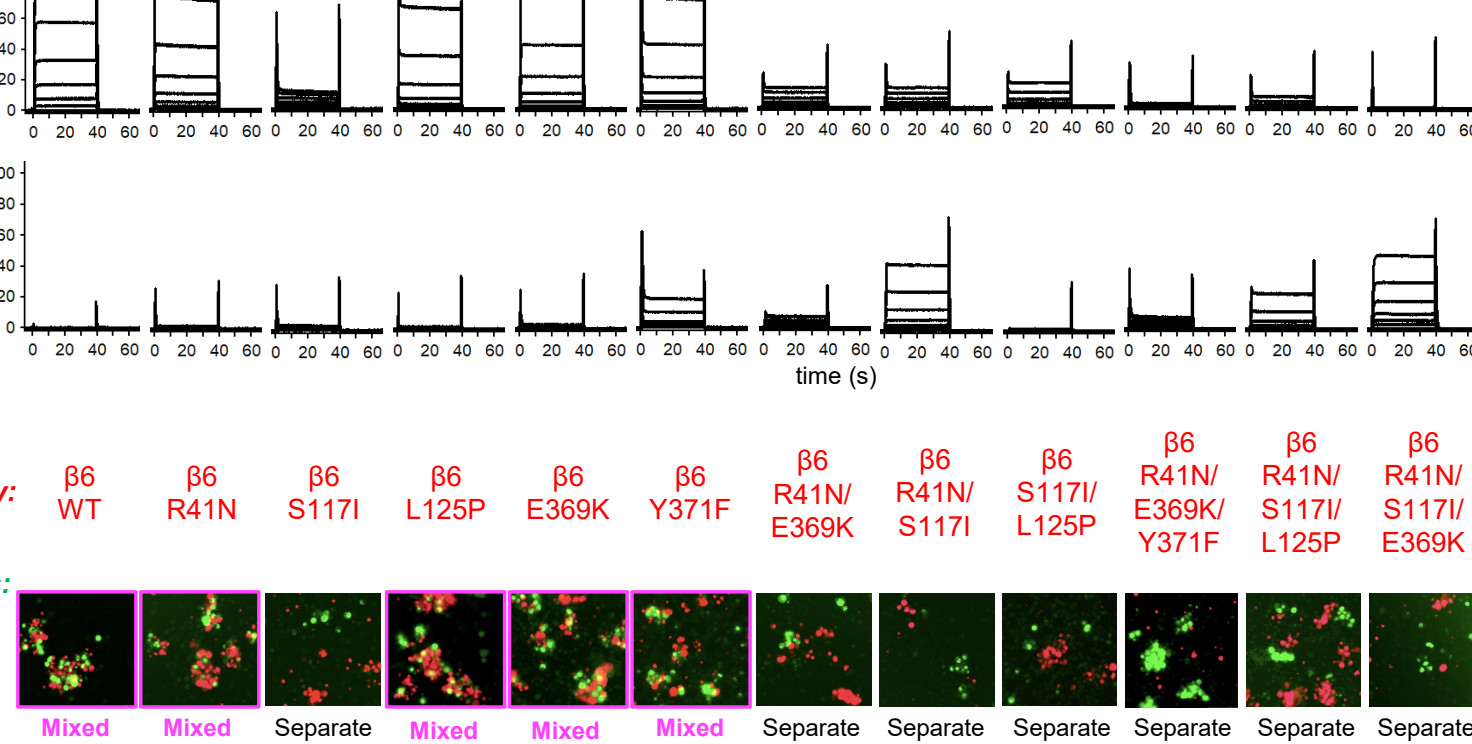

E369K/

Y371F

$\beta 6_{1-4}$
R41N/

S117I/

L125P/

$\beta 8_{1-4}$

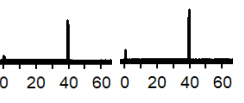

$\beta 8$ WT
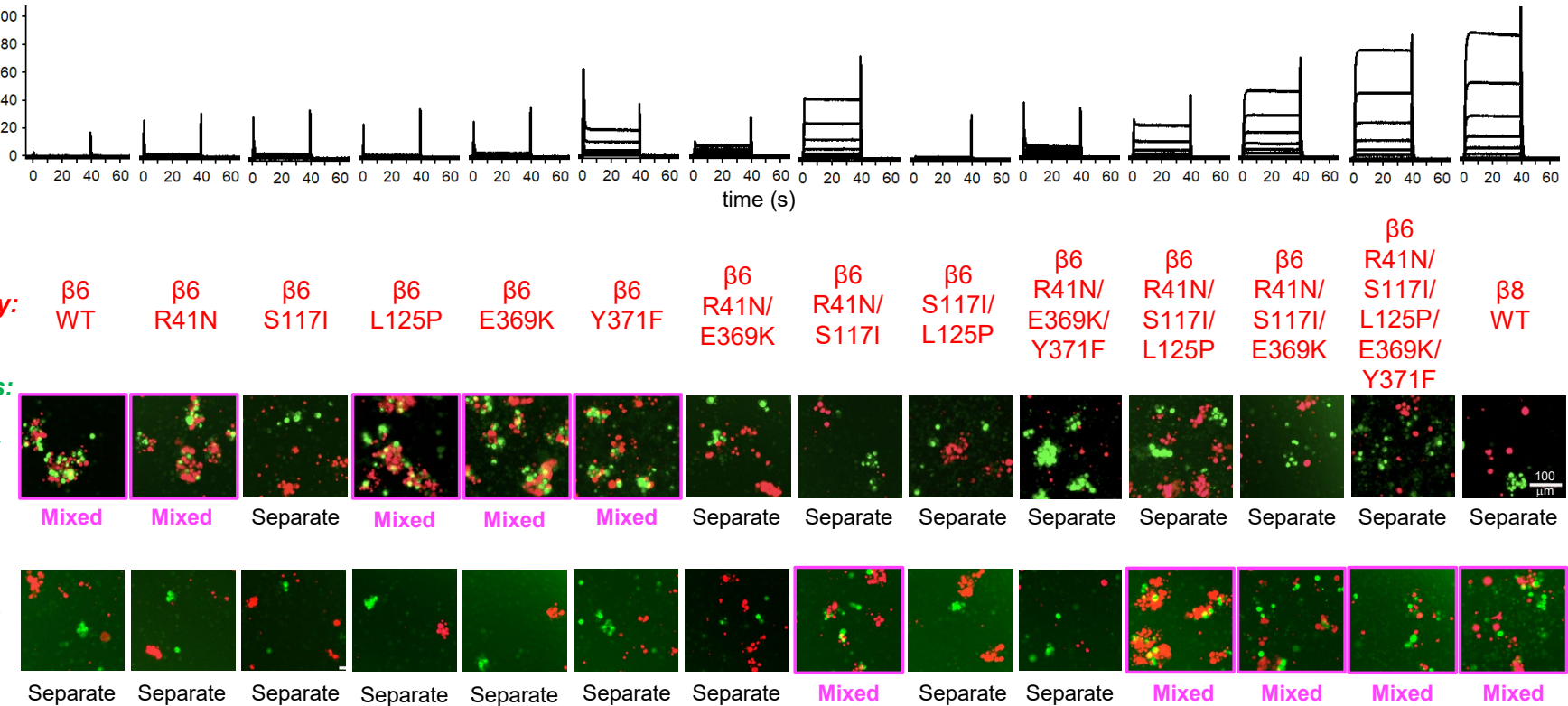

Figure 2 - figure supplement 2: Mutagenesis experiments reveal role in trans specificity for the five interfacial residue differences between close pair $\beta 6_{1-4}$ and $\beta 8_{1-4}$

A. Structural superposition of the $\beta 6_{1-4}$ and $\beta 8_{1-4}$ trans dimer crystal structures (PDBs: 5DZX and 5DZY) shown in ribbon depiction above, with close-up views of the trans interfacial regions containing the five interfacial residues that vary between $\beta 6_{1-4}$ and $\beta 8_{1-4}$ shown below. The two protomers forming the $\beta 6_{1-4}$ dimer are colored green and pale green respectively. The $\beta 8_{1-4}$ dimer is colored magenta/light pink. Bound calcium ions are shown as green spheres. Interfacial residue side chains are shown in the close-up views. The five variable residues are labelled with the $\beta 6_{1-4}$ amino acid given in green and the $\beta 8_{1-4}$ amino acid in magenta: R/N41 is in EC1; E/K369 and Y/F371 are in EC4; S/I117 is in EC2 and self-interacts at the trans dimer center of symmetry; and L/P125 is also in EC2.

B. SPR binding profiles of $\beta 6$ trans interface mutants converting $\beta 6_{1-4}$ to $\beta 8_{1-4}$ and the wild-type molecules (shown in columns) were flowed over surfaces coated with wild-type $\beta 6_{1-4}$ or wild-type $\beta 8_{1-4}$ (rows).

C. Results of the K562 co-aggregation assay where cells transfected with mCherry labeled $\beta 6$ and $\beta 8$ wild-types (WT) and the same trans-specificity mutants as in (B) were each mixed with cells transfected with mVenus labeled $\beta 6$ and $\beta 8$ wild-types (WT). Experiments where the red and green cells co-aggregate demonstrating interaction between the mCherry-labeled WT or mutant cPcdh and the mVenus-labeled WT cPcdh are labeled "mixed" and highlighted with magenta boxes. Scale bar, $100 \mu \mathrm{M}$. 


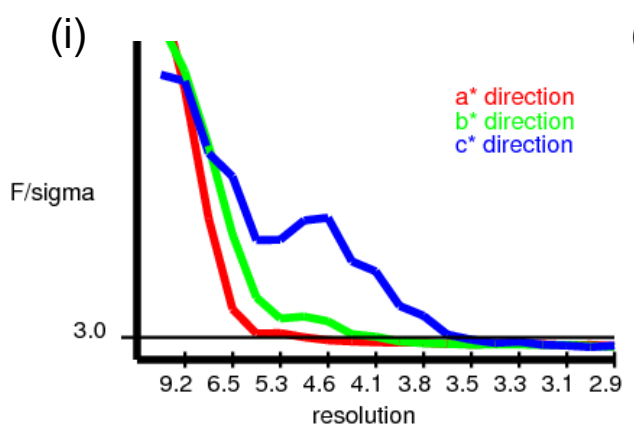

The recommended resolution limits along $\mathrm{a}^{*}, \mathrm{~b}^{*}, \mathrm{c}^{*}$ are 4.6 Ang 3.9 Ang 3.5 Ang These are the resolutions at which F/sigma drops below an arbitrary cutoff of 3.0 (ii)
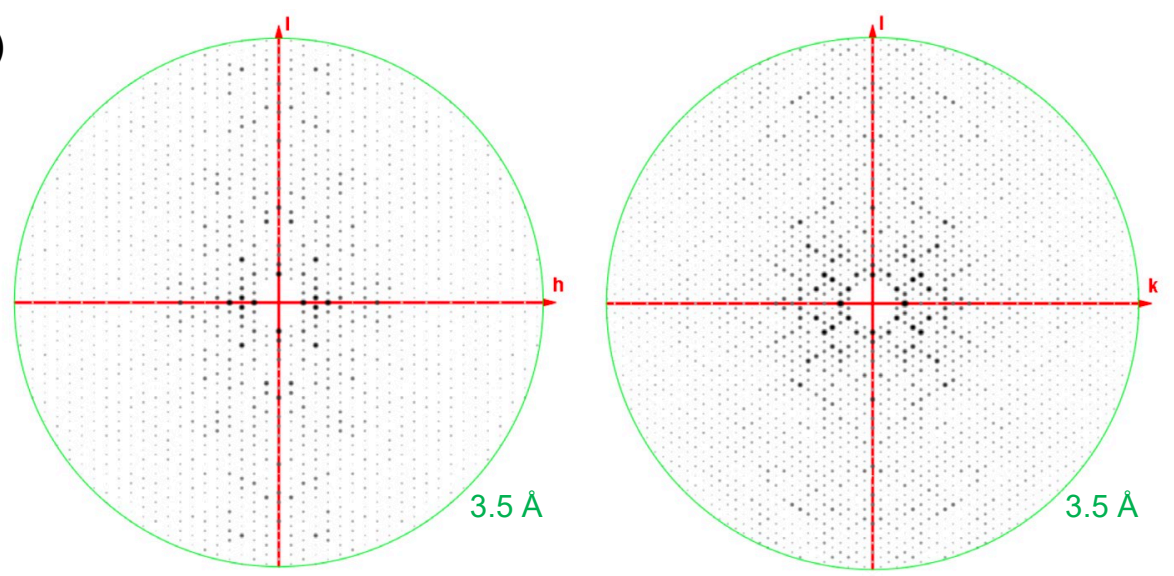

B $\quad \underline{ }$ C4 crystal form 1 trans dimer interface

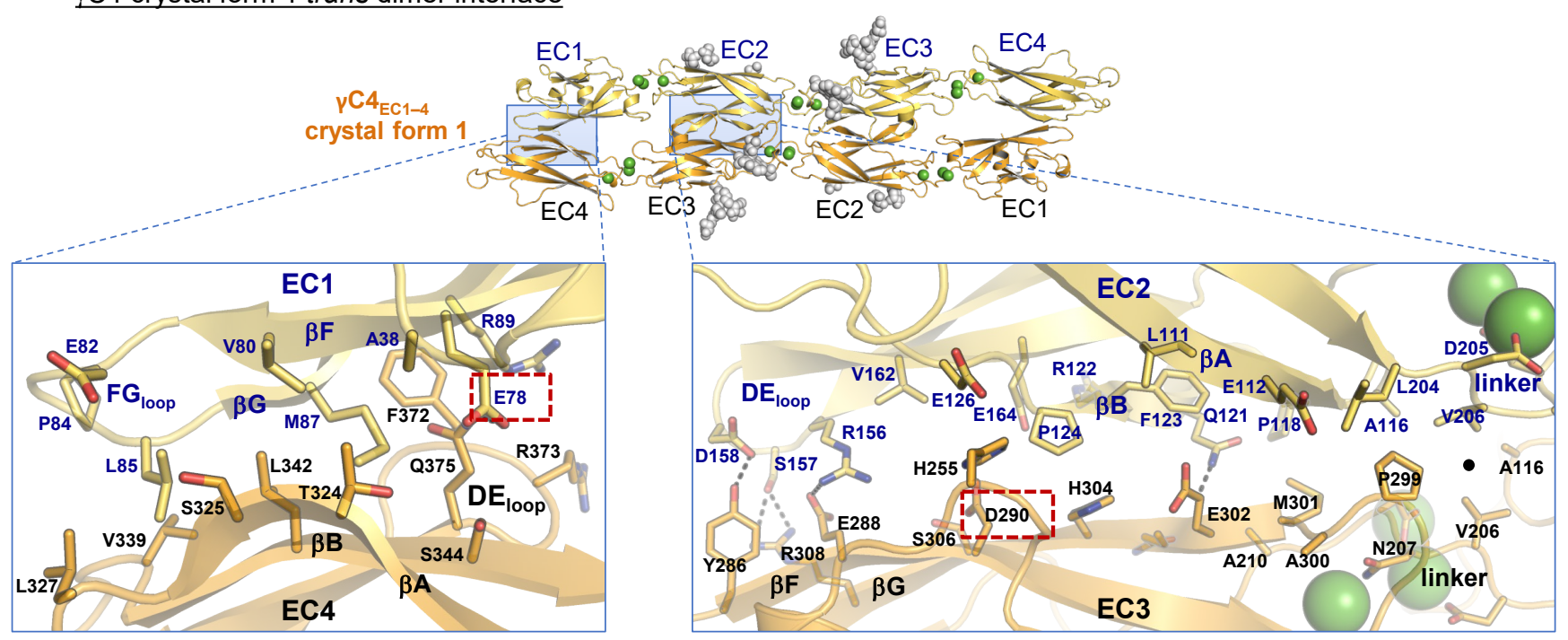

C

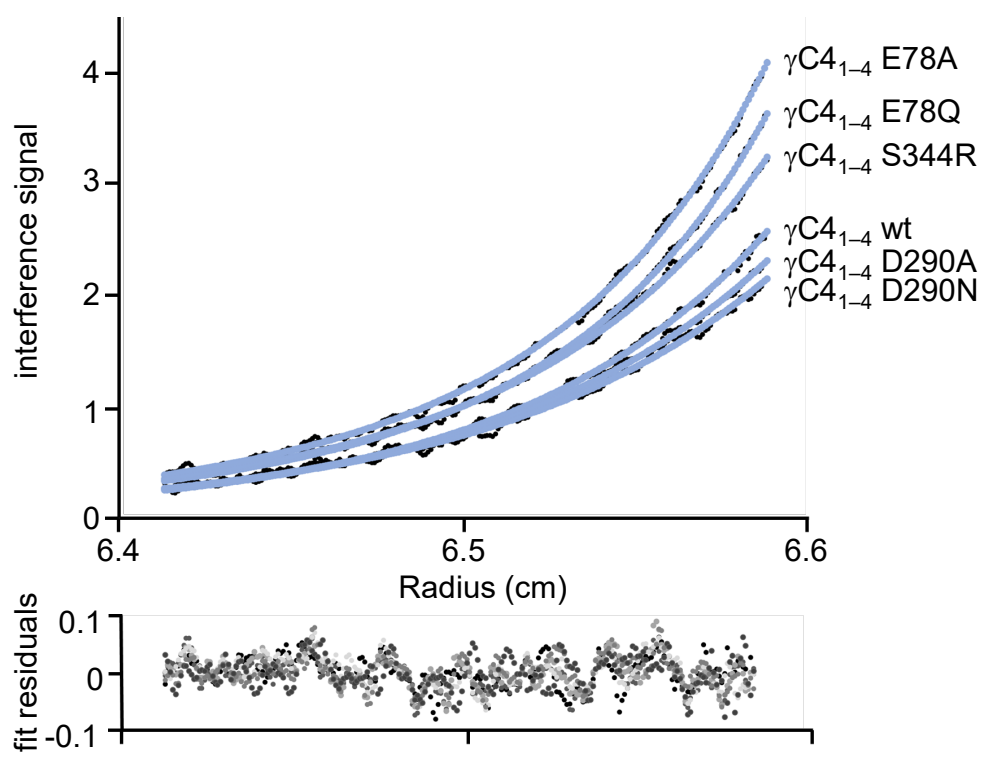

Figure 3-figure supplement 1: $\gamma \mathrm{C} 4$ trans dimer crystal structures and trans interface analysis

(A) Our crystallization experiments with $\gamma \mathrm{C}_{\mathrm{ECl}-4}$ yielded two distinct crystal forms the first of which showed significant $\mathrm{X}$ ray diffraction anisotropy. (i) UCLA Diffraction Anisotropy Server (Strong et al., 2006) plot shows the F/sigma by resolution along the $a^{*}, b^{*}$ and $c^{*}$ axes. (ii) Synthetic precession photographs of the X-ray diffraction in the $\mathrm{k}=0$ plane (left) and the $\mathrm{h}=0$ plane (right) showing the comparatively stronger/weaker diffraction.

(B) Close up views of the EC1:EC4 and EC2:EC3 interfacial regions from the first crystal form. One protomer in the symmetric dimer is colored yellow the other orange. Interfacial residues are labeled, side chains are shown in stick representation and dashed black lines depict potential interfacial hydrogen bond interactions. The two charged residues, E78 and D290, we selected for mutagenesis experiments to see whether they play a destabilizing role in the $\gamma \mathrm{C} 4$ trans interaction are marked with red dashed boxes. (C) Representative plot of AUC data for the wild type (wt) and mutant $\gamma \mathrm{C} 4 \mathrm{EC} 1-4$ molecules. Raw data are shown in black circles, and the non-linear fits to a monomer-to-dimer model are shown as blue lines. The residuals between the data and fits are shown in the plot below. Table detailing the oligomeric state and dissociation constants determined from the AUC data is shown in Figure 3. 
Figure 4-figure supplement 1

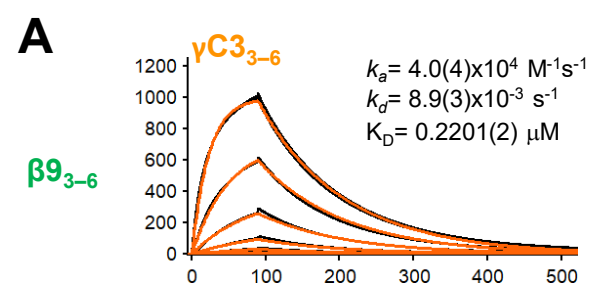

B

B
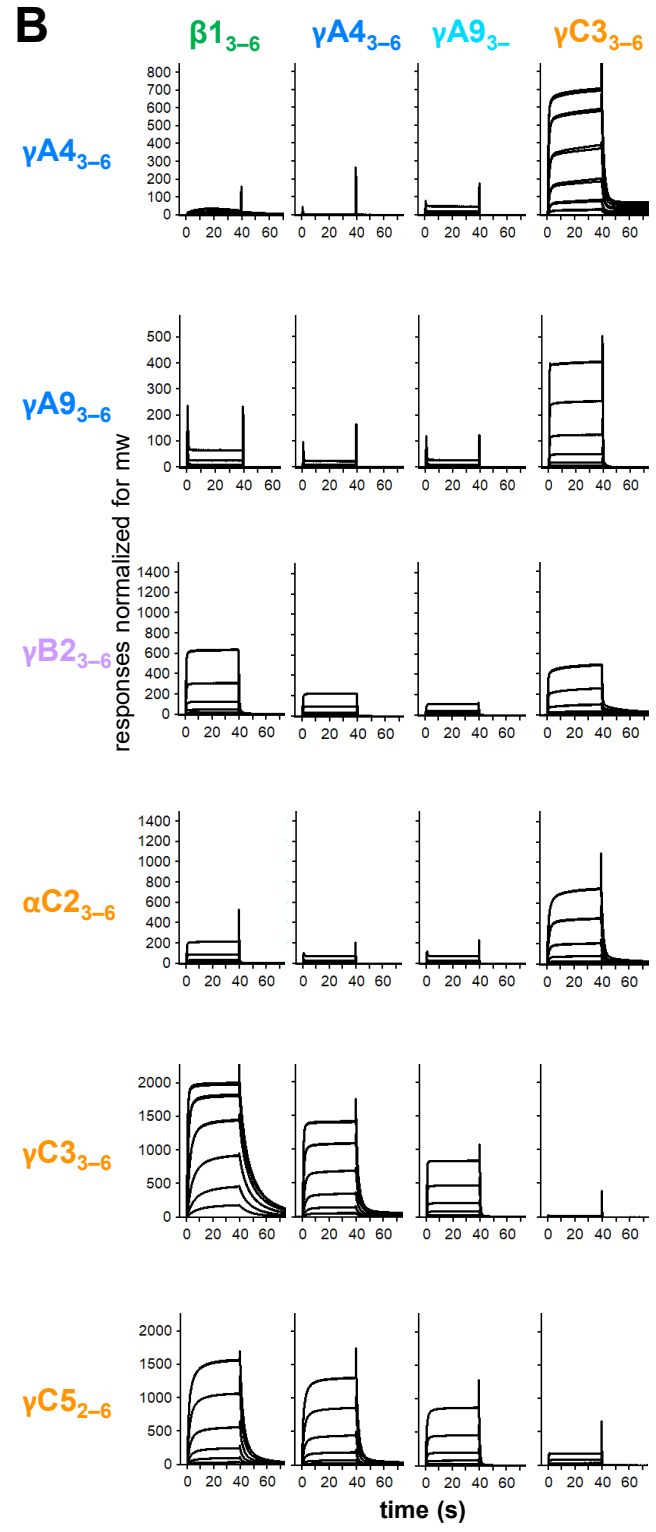
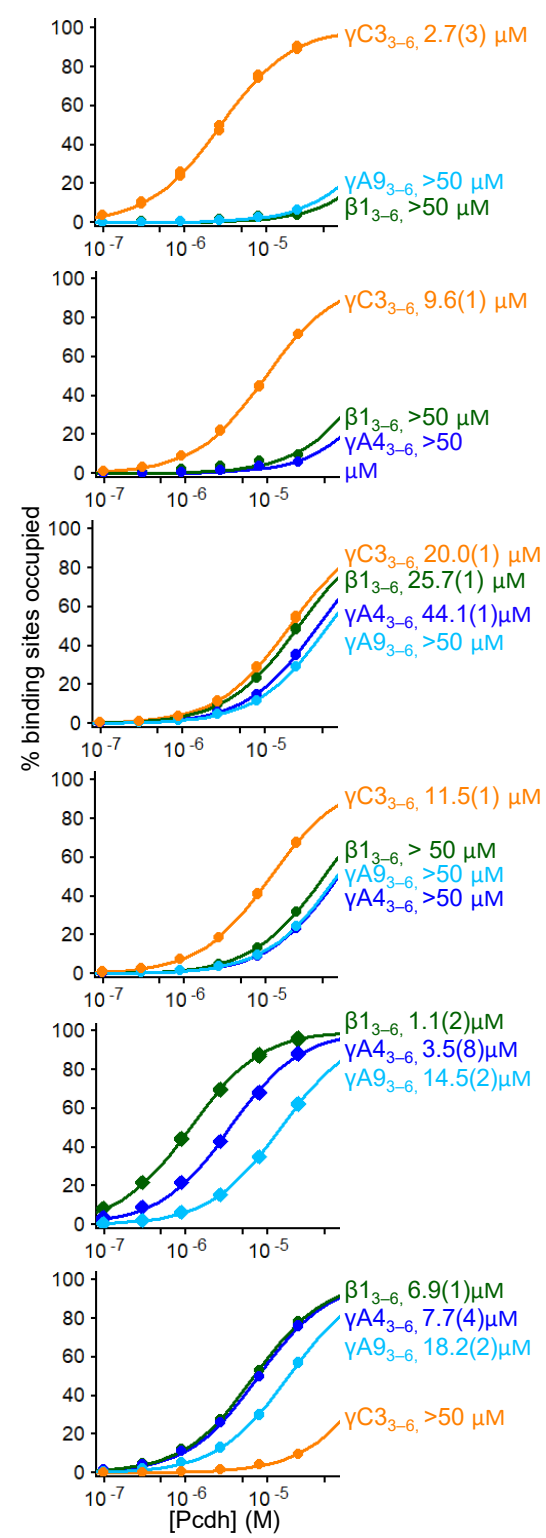
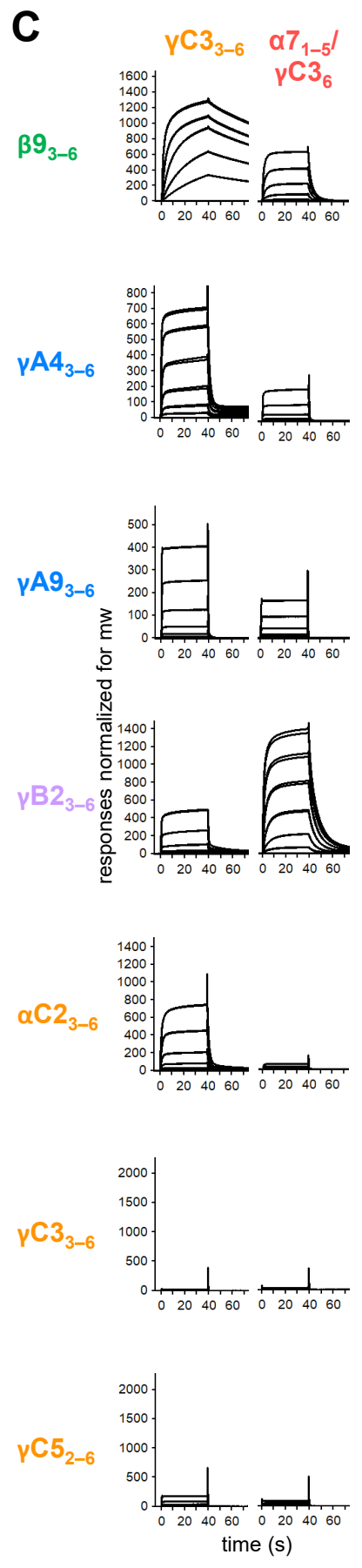

Figure 4-figure supplement 1: Calculation of cis interaction dissociation constants and the impact of an $\alpha$ Pcdh EC5 on family-wide cis interactions

(A) Kinetic binding analysis of $\gamma \mathrm{C}_{3-6}$ analyte binding over a $\beta 9_{3-6}$ covered surface. Data is shown in black, and the red traces represent the fit to an 1:1 binding model.

(B) Left, SPR binding profiles from Figure 4 for the four monomeric cis fragment analytes over all six cis fragment surfaces. Right, fit of the binding data for these four analytes to $1: 1$ binding isotherms to calculate $\mathrm{K}_{\mathrm{D}} \mathrm{S}$. $\gamma \mathrm{A}_{3-6}$ and $\gamma \mathrm{A} 9_{3-6}$ are monomeric and they are not included in the binding isotherms over their respective surface.

(C) SPR binding profiles for $\gamma \mathrm{C}_{3-6}$ (from Figure 4) and an $\alpha 7_{1-5} / \gamma \mathrm{C} 3_{6}$ chimera flowed over the immobilized cis fragment surfaces. Binding profiles for each surface are individually scaled as in Figure 4. 
Figure 4-figure supplement 2

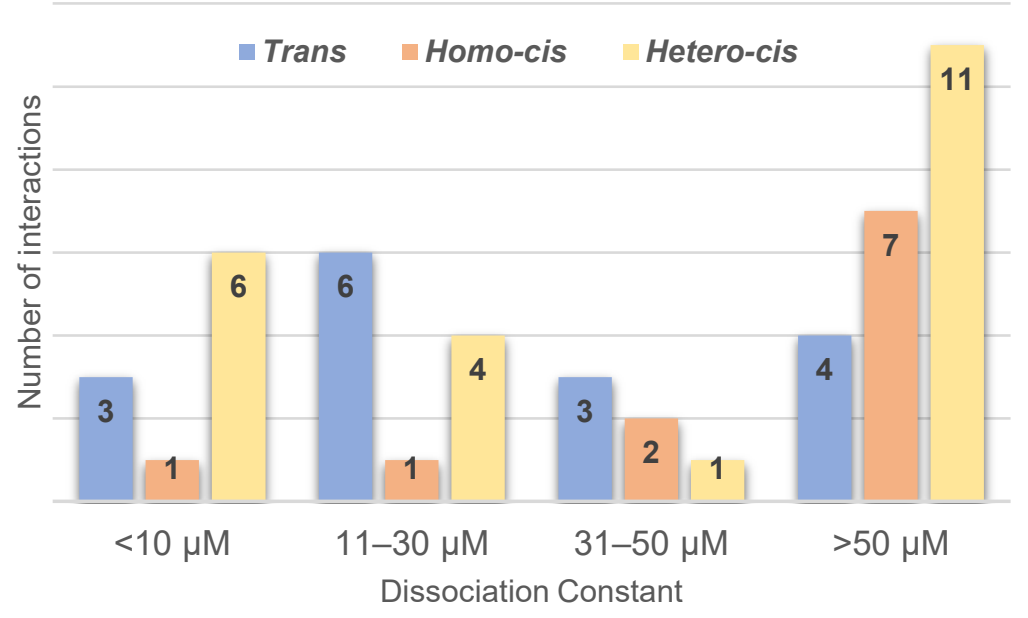

Figure 4-figure supplement 2: Range of $\mathrm{cPcdh}$ cis and trans Dissociation constants, $\mathrm{K}_{\mathrm{D}} \mathrm{s}$

Chart shows the cPcdh trans dimer, homophilic cis dimer, and heterophilic cis dimer interactions for which we have determined binding affinities divided into four subgroups based on their dissociation constant. The trans and homophilic cis dimer affinities were determined using AUC (Figure 2-source data 1 and Figure 4 -source data 1) and the heterophilic cis dimer affinities were determined using SPR (Figure 4B). Of the interactions in the $>50 \mu \mathrm{M}$ group one trans interaction and four homophilic cis interactions are monomeric in solution $(>500 \mu \mathrm{M}$ $\mathrm{K}_{\mathrm{D}}$ in AUC). Three of the 11 heterophilic cis interactions in the $>50 \mu \mathrm{M}$ group show no binding in our SPR experiments based on a 40 RU binding threshold. 
A
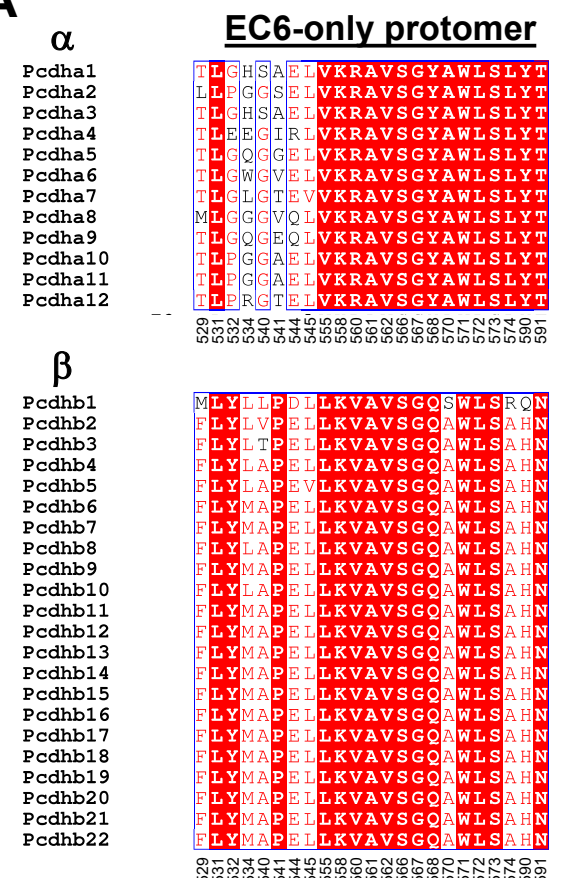

$\gamma \mathbf{A}$

Pcdhga1

Pcdhga2

Pcdhga 4

Pcdhga5

Pcdhga 6

Pcdhga7

Pcdhga8

Pcdhga 9

Pcdhgalo

Pcdhga11

Pcongar

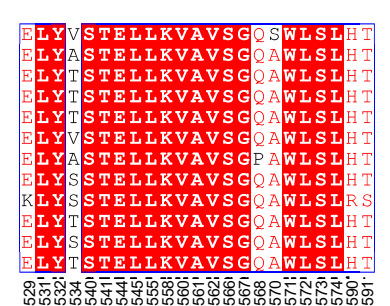

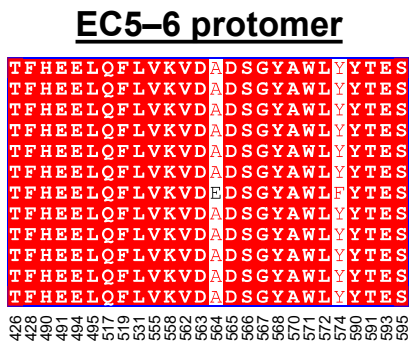

$\gamma \mathrm{B}$
Pcdhgb1
Pcdhgb2
Pcdhgb4
Pcdhgb5
Pcdhgb6
Pcdhgb7
Pcdhgb8

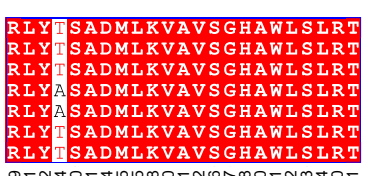

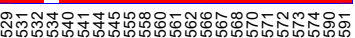

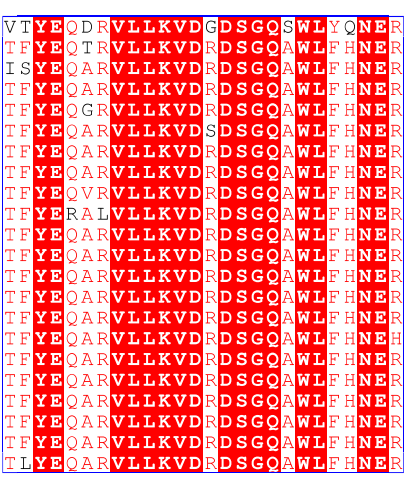

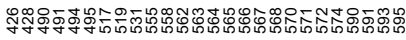

\section{C-types}

$$
\begin{aligned}
& \text { Pcdhac1 } \\
& \text { Pcdhac2 } \\
& \text { Pcdhgc3 } \\
& \text { Pcdhgc4 } \\
& \text { Pcdhgc5 }
\end{aligned}
$$

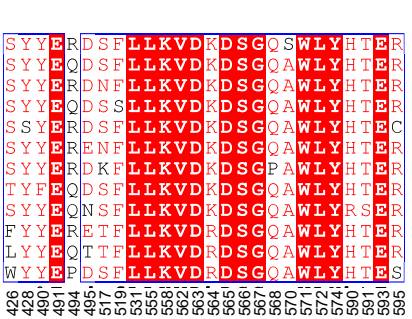

consensus $>70$
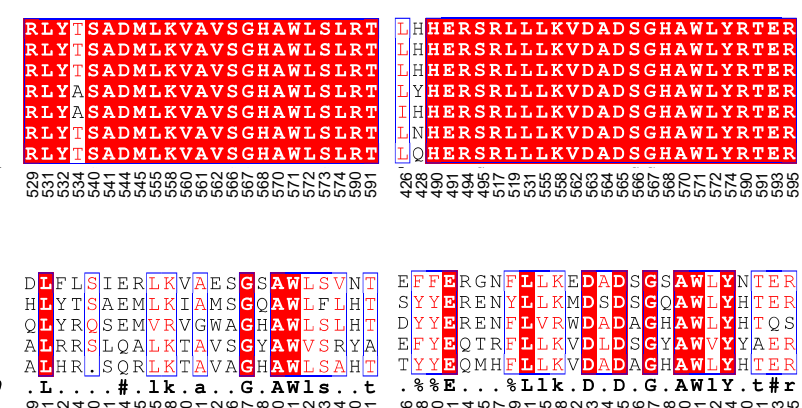

Figure 4-figure supplement 3: Amino acid sequence alignment reveals conservation of cis interfacial residues within the alternate $\mathrm{cPcdh}$ subfamilies

(A) Amino acid sequence alignments of cis interfacial residues from the EC6-only and EC5-6 surfaces for all 58 mouse cPcdhs subdivided by subfamily. Completely conserved residues are highlighted in red with white lettering. Residues 540 and 541 are included in the EC6-only alignments since the crystal structure of $\gamma \mathrm{A} 4$ EC36 (PDB: 5SZQ) revealed a distinct EC6 A-A'loop architecture to that observed in the $\gamma \mathrm{B} 2,4$, and 7 (PDBs: 5SZR, 6E6B, and 5V5X) cis fragment crystal structures that would place these residues in the EC6-only interface if maintained in cis interactions.

(B) Sequence logos based on the sequence alignment shown in (A) for the EC6-only cis interfacial residues from each of the five cPcdh subfamilies highlighting the similarities and conserved differences between the subfamilies. Residues 540 and 541 are included for all isoforms but greyed out for the non- $\gamma \mathrm{A}$ isoforms since their involvement may be $\gamma \mathrm{A}$-specific. NB: Previous studies have shown that $\alpha$-Pcdhs have an impaired EC6only interface (Thu et al., 2014; Goodman et al., 2017).

(C) Sequence logos for the EC5-6 cis interfacial residues from each of the five cPcdh subfamilies 


\section{Figure 5-figure supplement 1}

A $\underline{\underline{B} 77 \text { cis dimer interface }}$
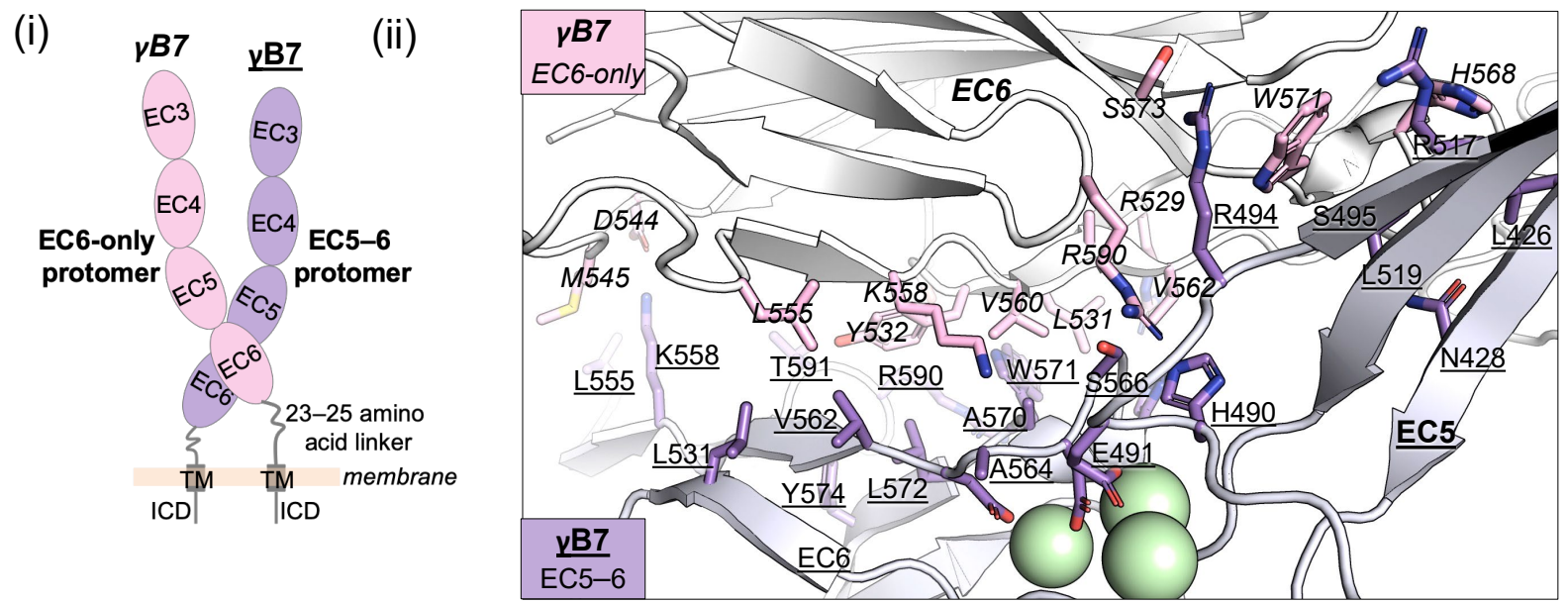

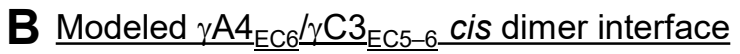

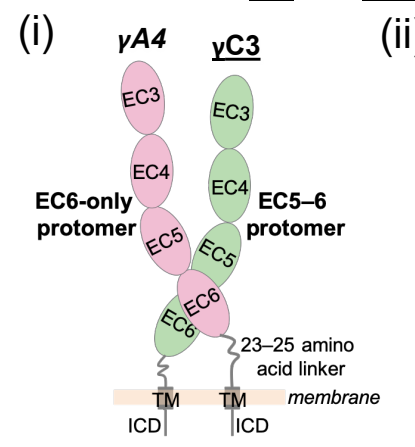

(ii)

$\gamma$ A specific A-A' loop (residues 540-544)

E544:

potential additional salt-bridge

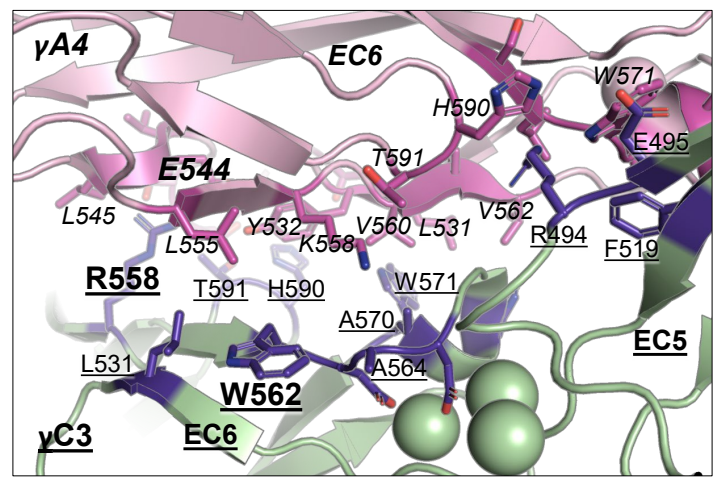

W562 increases hydrophobic surface area compared to $\gamma \mathrm{B} 7$ V562

C Modeled $\gamma \mathrm{C3}_{\mathrm{EC} 6} \underline{\gamma \mathrm{A} 4_{E C 5-6}}$ cis dimer interface

(i)

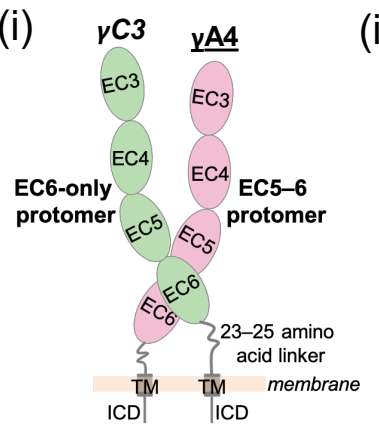

(ii)

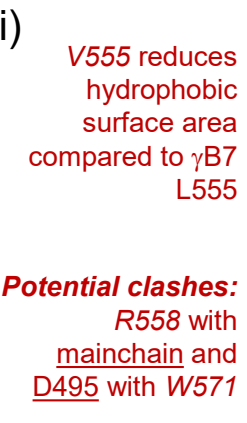

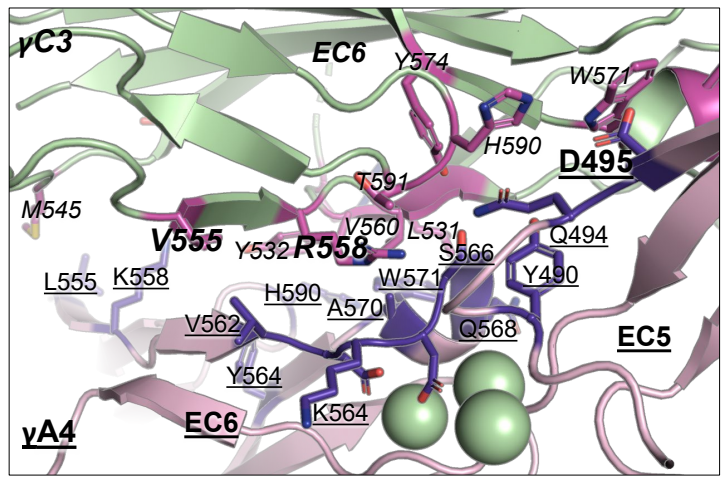

Reduced EC5 binding surface: Serine in place of W571-interacting hydrophobic residue at position 519

\section{Figure 5-figure supplement 1: Structure-guided sequence analysis of $\gamma \mathrm{A} 4$ and $\gamma \mathrm{C3}$ cis interactions}

(A) (i) Schematic of the asymmetric $\gamma \mathrm{B} 7_{\mathrm{EC} 3-6}$ cis dimer crystal structure. (ii) Close-up view of the $\gamma \mathrm{B} 7$ cis interface: Interfacial residue side chains are shown in pink for the EC6-only protomer and purple for the EC5-6 protomer. Bound calcium ions are shown as green spheres.

(B) (i) Schematic of the $\gamma \mathrm{A}_{\mathrm{EC} 6} / \gamma \mathrm{C} 3_{\mathrm{EC} 5-6}$ cis dimer. (ii) Model of the $\gamma \mathrm{A}_{\mathrm{EC} 6} / \gamma \mathrm{C} 3_{\mathrm{EC} 5-6}$ cis dimer interaction generated using structural alignment of EC6 from the monomeric $\gamma \mathrm{A} 4 \mathrm{EC} 3-6$ crystal structure (PDB 5SZQ) to the $\gamma \mathrm{B} 7 \mathrm{EC} 3-6$ cis dimer structure for the EC6only side and computational mutagenesis of $\gamma \mathrm{B} 7$ to $\gamma \mathrm{C} 3$ selecting the best-fit rotamer (without energy minimization) for the EC5-6 side. The model suggests that this will be the preferred orientation for the $\gamma \mathrm{A} 4 / \gamma \mathrm{C} 3$ cis dimer interaction. Favorable residue differences between $\gamma \mathrm{B} 7$ from (A) and $\gamma \mathrm{A} 4 / \gamma \mathrm{C} 3$ in this orientation are noted in green. Please note the model shown here is only used for hypothesis generation, since it is unlikely to be completely accurate.

(C) (i) Schematic of the $\gamma \mathrm{C} 3_{\mathrm{EC} 6} / \gamma \mathrm{A} 4_{\mathrm{EC} 5-6}$ cis dimer. (ii) Model of the $\gamma \mathrm{C} 3_{\mathrm{EC} 6} / \gamma \mathrm{A} 4_{\mathrm{EC} 5-6}$ cis dimer generated using computational mutagenesis of $\gamma \mathrm{B} 7$ to $\gamma \mathrm{C} 3$ selecting the best-fit rotamer (without energy minimization) for the EC6-only side and structural alignment of EC5-6 from the $\gamma \mathrm{A} 4$ EC3-6 crystal structure to the $\gamma \mathrm{B} 7 \mathrm{EC} 3-6$ cis dimer structure for the EC5-6 side. The model suggests that this orientation for the $\gamma \mathrm{A} 4 / \gamma \mathrm{C} 3$ cis dimer interaction will be disfavored. Unfavorable residue differences between $\gamma \mathrm{B} 7$ and $\gamma \mathrm{A} 4 / \gamma \mathrm{C} 3$ in this orientation are noted in red. Please note the model shown here is unlikely to be completely accurate and is simply for hypothesis generation. 
Figure 5-figure supplement 2
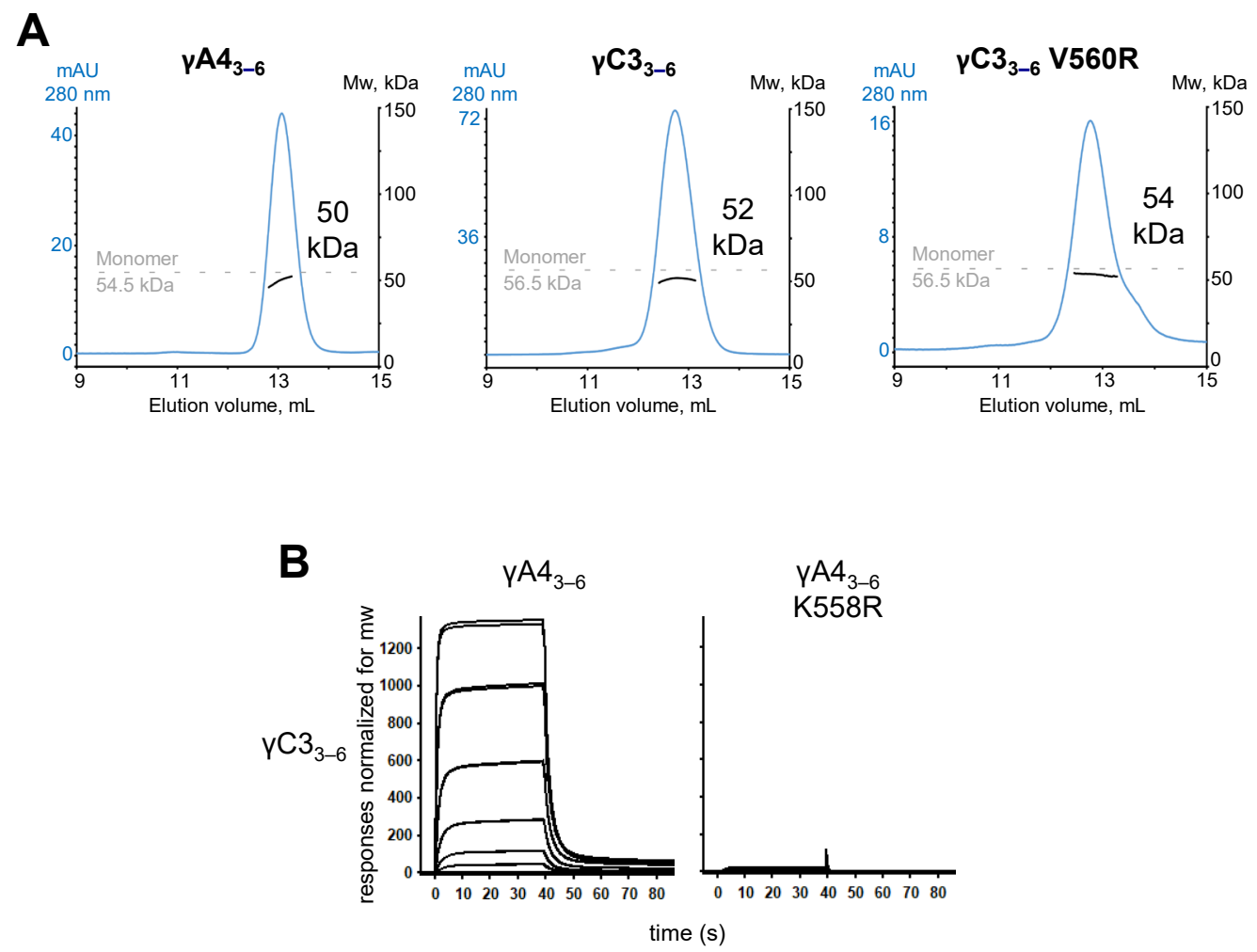

Figure 5-figure supplement 2: $\gamma \mathrm{A} 4$ and $\gamma \mathrm{C3}$ cis-fragments behave as monomers in SEC-MALS and mutating $\gamma \mathrm{A} 4$ to make it more like $\gamma \mathrm{C} 3$ prevents $\gamma \mathrm{A} 4 / \gamma \mathrm{C} 3$ cis-heterodimerization

(A) SEC-MALS data for wild-type $\gamma \mathrm{A}_{3-6}$, wild-type $\gamma \mathrm{C}_{3-6}$, and $\gamma \mathrm{C}_{3-6} \mathrm{~V} 560 \mathrm{R}$ showing all three molecules are monomeric in SEC-MALS, consistent with their behavior in sedimentation equilibrium AUC. Plots show size exclusion absorbance at $280 \mathrm{~nm}$ trace in blue (left axis), molecular weight of the eluant peak in black (right axis), and the monomer molecular weight of $\gamma \mathrm{A}_{3-6}$ or $\gamma \mathrm{C} 3_{3-6}$ measured by mass spectrometry $-54.5 \mathrm{kDa}$ and $56.5 \mathrm{kDa}$ respectively - as dashed grey lines. Average molecular weight of the molecules in the eluant peaks are labeled. (B) SPR binding profiles for $\gamma \mathrm{A} 4_{3-6}$ wild type and $\gamma \mathrm{A} 4_{3-6}$ with $\gamma \mathrm{C} 3$-like $c i$ interface mutation K558R flowed over immobilized wild-type $\gamma \mathrm{C}_{3-6}$. Loss of $\gamma \mathrm{C}_{3-6}$ interaction in the presence of the K558R mutation is consistent with the SEC-MALS results shown in Figure 5. 